\title{
Role of taurine, its haloamines and its IncRNA TUG1 in both inflammation and cancer progression. On the road to therapeutics? (Review)
}

\author{
STELLA BALIOU $^{1}$, ANTHONY M. KYRIAKOPOULOS ${ }^{2}$, \\ DEMETRIOS A. SPANDIDOS ${ }^{3}$ and VASSILIOS ZOUMPOURLIS ${ }^{1}$ \\ ${ }^{1}$ National Hellenic Research Foundation, 11635 Athens; ${ }^{2}$ Nasco AD Biotechnology Laboratory, 18536 Pireus; \\ ${ }^{3}$ Laboratory of Clinical Virology, Medical School, University of Crete, Heraklion 71003, Greece
}

Received June 12, 2020; Accepted July 14, 2020

DOI: $10.3892 /$ ijo. 2020.5100

\begin{abstract}
For one century, taurine is considered as an end product of sulfur metabolism. In this review, we discuss the beneficial effect of taurine, its haloamines and taurine upregulated gene 1 (TUG1) long non-coding RNA (lncRNA) in both cancer and inflammation. We outline how taurine or its haloamines (N-Bromotaurine or $\mathrm{N}$-Chlorotaurine) can induce robust and efficient responses against inflammatory diseases, providing insight into their molecular mechanisms. We also provide information about the use of taurine as a therapeutic approach to cancer. Taurine can be combined with other chemotherapeutic drugs, not only mediating durable responses in various malignancies, but also circumventing the limitations met from chemotherapeutic drugs, thus improving the therapeutic outcome. Interestingly, the lncRNA TUG1 is regarded as a promising therapeutic approach, which can overcome acquired resistance of cancer cells to selected strategies. In this regard, we can translate basic knowledge about taurine and its TUG1 IncRNA into potential therapeutic options directed against specific oncogenic signaling targets, thereby bridging the gap between bench and bedside.
\end{abstract}

\section{Contents}

1. The role of taurine in inflammation

2. Formation of taurine haloamines

3. Anti-microbial properties of taurine haloamines

4. Anti-inflammatory and anti-oxidant properties of taurine haloamines

Correspondence to: Dr Vassilios Zoumpourlis, National Hellenic Research Foundation, 48 Vas. Konstantinou Avenue, 11635 Athens, Greece

E-mail: vzub@eie.gr

Key words: taurine, taurine haloamines, taurine upregulated gene 1 ncRNA, inflammation, cancer
5. Therapeutic perspectives and clinical studies

6. Significance of lncRNA TUG1 lncRNA in cancer

7. The association of lncRNA TUG1 and chemoresistance

8. The role of taurine or lncRNA TUG1 as a prognostic marker

9. Conclusions

\section{The role of taurine in inflammation}

Taurine (2-aminoethanesulfonic acid) is a non-essential amino acid that is abundant in all mammalian tissues. Taurine is essential for cell growth of renal, neural, and cardiac cells, preventing death procedures $(1,2)$. Taurine plays a significant role in homeostasis because it is involved in the regulation of the following processes: cell volume regulation, osmoregulation, protein phosphorylation, membrane stability, bile acid conjugation, neuromodulation, maintenance of calcium concentration, and detoxification of xenobiotics (3). The anti-oxidant and anti-inflammatory properties of taurine constitute the main mechanisms that account for its cytoprotection $(3,4)$.

Taurine accumulates in phagocytes (both neutrophils and macrophages) as well as in inflammatory lesions, illustrating its potential significance in innate immunity (5). It has been reported that taurine concentration can reach $50-70 \%$ in neutrophilic granulocytes, lymphocytes and monocytes (5-7). The contribution of taurine to the immune surveillance relies on the anti-oxidant properties of taurine (8) and its membrane-stabilizing capacity (9). For example, experimental evidence has highlighted that taurine mainly exerts its anti-oxidant activity through inhibition of sodium arsenite-induced apoptosis in neutrophils (10). Taurine has a protective role, sustaining the phagocytic ability of neutrophils independently the stimulus including age (11) or hyperlipidemia (12). Besides, some reports have highlighted that taurine exerts its beneficial effect on leukocytes, via alleviating the oxidative stress $(13,14)$. The pleiotropic nature of taurine is not tightly associated with the anti-oxidant properties, but it is also related to its membrane-stabilizing capacity in lymphocytes (15). In this direction, the anti-oxidant nature of 
taurine has been shown to account for preserving the viability of human lymphocyte-derived cultured lymphoblastoid cells, protecting against oxidant-induced damage caused by ferrous sulfate and ascorbate (15).

Taurine is regarded as a promising agent against numerous types of inflammatory injury (inflammatory bowel disease, pancreatitis, and gastric mucosal injury), due to its immunoregulatory importance $(16,17)$. Taurine is effective against various acute inflammation related diseases, including spinal cord injury (18), hepatic ischemia-reperfusion (19), lung injury $(20,21)$, ischemic stroke (22), and trinitrobenzene sulfonic acid (TNBS)-induced colitis in the rat (23), lipopolysaccharide (LPS)-induced acute lung injury in sheep (20) and dextran sulfate sodium (DSS)-induced colitis $(24,25)$. In these conditions, the anti-inflammatory action of taurine is usually attributed to its antioxidant effect, which is manifested by inhibiting lipid peroxidation (LPO) (16). The anti-inflammatory effect of taurine has also been attributed to the reduced secretion of interleukins (ILs) (such as IL-8), as shown by experiments in Caco-2 cells, without any participation of polymorphonuclear leukocytes (24). Accordingly, there is a growing body of preclinical data that demonstrates the anti-inflammatory effects of taurine in both neural and systemic inflammation including cardiovascular disease (26), traumatic brain injury (27), liver/gallbladder disease (28), lung injury (29), diabetes (30), cataract (31). As a result, taurine fulfills the necessary criteria to participate in the regulatory network of an immune response.

Moreover, the immune-regulatory effect of taurine has been validated through studies examining the consequences of a taurine deficiency. When taurine elimination arose in cats, the immune landscape was reorganized. In particular, taurine-deficient cats presented significant leukopenia, decreased respiratory burst in neutrophils, and depletion of cells from B cell areas in lymph nodes and splenic follicle centers (32). Apart from leukopenia, taurine deficiency proved to cause functional defects of the neutrophils and decreased phagocytosis of microorganisms such as Staphylococcus epidermis $(5,33)$. Conversely, taurine was reported to mediate its ameliorative effect on age-related decline in the proliferative ability of lymphocytes through increasing calcium levels. In particular, the effect of taurine was more potent on T-cells, that were more susceptible to age-related decline in proliferation than B lymphocytes (32). The effect of taurine on lymphocyte function was substantiated through its chaperoning role concerning MHC class II antigen expression (33). The aforementioned data were evaluated given that T-cell proliferation is mediated independently of the age-related alteration in taurine transport (34).

Besides, it is important to be noted, taurine biosynthesis has been outlined to be divided into the oxidation of cysteine to cysteine sulfinic acid followed by the decarboxylation to hypotaurine, with the subsequent oxidation to taurine (35). In this sense, the significance of taurine has been proven in the immune system through the elimination of cysteine sulfinic acid decarboxylase (CSAD), which is crucial for the conversion of cysteine sulfinic acid to hypotaurine (35). In particular, it has been observed that taurine concentration was significantly higher in the splenocytes and macrophages from
CSAD knock-out (KO) mice compared to those encountered in the liver and plasma from CSAD KO mice $(7,36)$, implying its significance in the immune system.

Beneficial effect of taurine in various cancers (in vitro and in vivo). Cancer is a direct consequence of gene mutations that arise in a multistep process, enabling cancer cells to possess a sustained replicative potential (16). Vogelstein declared that 'The revolution in cancer research can be summed up in a single sentence: Cancer is, in essence, a genetic disease' (37). Tumorigenesis comprises a series of events, in which excessive reactive oxygen species (ROS) formation is the determinant force for cancer development (38). In line with this, many anti-oxidants including methionine, cysteine, and taurine have been identified to display strong potential of minimizing oxidative injury in cancer $(32,39)$. On the contrary, cancer cells have been reported to constitutively express low ROS levels and high antioxidant responses during tumor progression (40). This specific vulnerability of various tumor cells is termed 'non-oncogene dependency' (38). In keeping with this observation, small molecular weight pro-oxidant drugs have been shown to cause an oxidative burst in cancer cells harboring low oxidative status, with the ultimate aim of eradicating them (41).

Since taurine exerts a strong anti-inflammatory action, the functional significance of taurine has been presented in orchestrating the landscape of tumor cells. Meeting this objective, many research groups have illustrated the anti-cancer impact of taurine, providing insights into its molecular mechanisms. Taurine functions as a redox-directed agent to specifically target tumor cells, raising the possibilities to achieve drug selectivity without off-target toxicity. Several cases have proved that taurine displays strong growth-inhibitory effect on multiple cancer types including colon cancer $(42,43)$, lung cancer (44), hepatocarcinoma (30), pancreatic cancer (45), glioma (46), melanoma (47) breast cancer (48-51), nasopharyngeal carcinoma (NPC) $(52)$, prostate cancer $(53,54)$ and ovarian cancer $(55,56)$.

Regarding the molecular mechanisms underlying the anti-cancer effect of taurine, it has been proposed that the effect of taurine on tumor cells can be either cytostatic (i.e., cell growth suppression) or cytotoxic (i.e., direct toxic effect). The anti-cancer effect of taurine is mainly mediated through multiple molecular mechanisms. Firstly, taurine exerts a growth-inhibitory effect through its antioxidant nature (57). In most cases, the main molecular mechanism underlying the anti-cancer effect of taurine relies on modulating multiple signaling cascades $(42,50,51,58-60)$, through its anti-oxidant capacity (61-65). For example, taurine has been reported to protect cells from oxidant-induced injury by neutralizing insults derived from strong oxidant and cytotoxic agents (66). As a further example, taurine has been proposed as an effective antioxidant, preventing the accumulation of ROS in tumor cells, thereby compromising cancer progression (67). Secondly, taurine ameliorates the efficacy of chemotherapeutic drugs, minimizing their toxicity $(68,69)$. It has been pointed out that taurine supplementation overcomes chemotherapy-induced complications, probably owing to its antioxidant capacity (70-75). In particular, taurine shows strong potential to attenuate toxic side effects 
of classic chemotherapeutic drugs [doxorubicin (DOX), 5-fluorouracil (5-FU), cisplatin, tamoxifen (TAM)], thereby enhancing their therapeutic efficacy $(61,74,76-78)$. In this sense, taurine is crucial to expand the therapeutic window of selected anti-tumor drugs, thereby optimizing the therapeutic efficacy of drugs. Thirdly, taurine plays a significant role in the immune rejection of cancer cells by enhancing immune surveillance (31). Fourth, taurine imparts its preventive action on cancer cells through the induction of apoptosis (42). In support, studies have shown that taurine triggers apoptosis in colon cancer (42), breast cancer (50), and hepatocarcinoma (60). For example, the apoptotic effect of taurine is accomplished by up-regulating the expression of the p53 transcription factor, while down-regulating the expression of anti-apoptotic proteins such as B-cell lymphoma 2 (BCL-2) (42). In another case, taurine has been proved to display its anti-neoplastic activity through the induction of apoptosis in NPC (52). The mechanism underlying the apoptotic effect of taurine is based on stimulating endoplasmic reticulum stress and inactivating the protein kinase B (Akt) signaling pathway (52). Besides, tumors are characterized by a permissive microenvironment that favors the induction of neo-angiogenesis for maintaining the supply of oxygen and nutrients (16). In this context, the anti-cancer activity of taurine has been illustrated to be elicited through the inhibition of tumor neovascularization and the induction of cytotoxicity on endothelial cells. For example, taurine has been proved to downregulate matrix metalloproteinase 2 (MMP-2), and to upregulate of $\mathrm{N}$-acetylgalactosaminytransferase, thereby preventing the increased invasiveness of cancer cells from primary site through bloodstream to other sites, in response to ionizing radiation (79). According to those viewpoints, a comprehensive in-depth analysis regarding the molecular mechansims of taurine underlying its therapeutic efficacy against distinct cancer types was outlined.

In breast cancer, epidemiological studies have suggested that an anti-oxidant enriched diet may be crucial to reducing the emergence of breast cancer (80). In this context, the group of Garmire used blood-based-metabolomics in conjunction with RNA-Seq-based on The Cancer Genome Atlas (TCGA) breast cancer data and highlighted that the taurine metabolic pathway is an important regulatory pathway among eight others, enabling the diagnosis of breast cancer occurrence in a personalized manner (81). Researchers have also used high-resolution magic angle spinning magnetic resonance spectroscopy (HR-MAS MRS) coupled with the relative principal component analysis, in biological samples of breast cancer patients, proving that small values for taurine were detected in breast cancer patients with metastasis compared to healthy patients (82). Similarly, four groups of female patients were recruited and were divided as follows: i) 50 diagnosed patients with breast cancer subjected to surgery; ii) 10 female patients with benign breast cancer signs; iii) 5 females equipped with high predisposition to breast cancer, due to their family history; and iv) 20 healthy women who were used as control samples to evaluate the diagnostic importance of taurine in Egyptian patients with breast cancer (83). Following the evaluation of female patients with breast cancer in various stages, taurine levels appeared to be reduced in the serum of patients with a high risk of breast cancer, providing a clue for the predisposition of women to breast cancer or the early diagnosis of females with early malignant lesions due to taurine detection (83).

In particular, the prognostic significance of taurine was confirmed in the serum of patients with breast cancer, because serum taurine levels were reduced in the breast cancer group and were tightly linked to tumor angiogenesis, as evidenced by reduced expression levels of angiogenesis markers [vascular endothelial growth factor (VEGF), CD31] and apoptotic markers [tumor necrosis factor- $\alpha$ (TNF- $\alpha$ ), caspase-3] (83). Interestingly, females with positive family history and women with benign breast lesions presented taurine levels ranging from 40 to $57 \mu \mathrm{mol} / 1$ and from 18 to $31 \mu \mathrm{mol} / 1$, respectively. In contrast, healthy women presented taurine range from 46 to $70 \mu \mathrm{mol} / 1$. It was highlighted that minimal taurine value was found in women with high susceptibility to breast cancer, proposing that minimal taurine value of high-risk group did not exceed the lower limit recorded in control healthy group (83).

Apart from the diagnostic and prognostic importance of taurine, a wide range of tumor cell lines and mouse models harboring mammary carcinogenesis have been employed to examine the cytotoxic effect of taurine on breast cancer. Initial experiments proved the beneficial impact of taurine on nude mice bearing breast cancer xenografts (50). The underlying molecular mechanism of taurine was based on inducing the mitochondrial cell death pathway, as shown by increased expression levels of p53-upregulated modulator of apoptosis (PUMA), irrespective of the p53 genetic profile (50). In 2,4-dimethoxybenzaldehyde (DMBA)-induced mammary carcinogenesis, the therapeutic impact of taurine emerged by inhibiting the migration of breast cancer cells through its strong antioxidant efficacy (59). In particular, the anti-oxidant effect of taurine relied on its capacity to hinder mitochondrial LPO and to normalize citric acid cycle enzyme expression, thus augmenting electron transport chain complexes and delaying electron cleavage responsible for the accumulation of ROS (59). In this context, taurine was proved to exert a strong anti-neoplastic effect on rats harboring mammary carcinogenesis, through its interference with energy metabolism of rats not only by reducing breast cancer incidence, but also forestalling breast cancer progression (84). The metabolic pattern of taurine-treated tumor-bearing rats was distinguished from that of tumor-bearing rats without taurine treatment, as shown by experiments in the model of DMBA-induced mammary carcinogenesis (84). In particular, taurine-supplemented tumor-bearing rats presented remarkable differences in 23 metabolites which participated in metabolic pathways of the urea cycle, Krebs cycle, protein synthesis, aspartic acid metabolism, alanine metabolism, ammonia circulation, and the malic acid-aspartic acid shuttle, compared to normal matched group (84). Interestingly, the plasma concentrations of fumarate, malate, citrate, $\alpha$-ketoglutarate, and pyruvate were detected to be lower in the taurine-supplemented breast cancer group relative to those derived from normal matched group (84). As a result, the antitumor activity of taurine was partially ascribed to the inhibition of aerobic glycolysis and the downregulation of enzymes involved in Krebs cycle (84). The beneficial impact of taurine was attributed to the interference with energy metabolism of breast cancer cells. 
Taurine has also exhibited its favorable effect against mammary carcinogenesis through its regulatory effect on the extracellular matrix (ECM), thus attenuating breast cancer recurrence. The therapeutic efficacy of taurine was ascertained in either estrogen receptor-dependent breast cancer cells (MCF-7) or estrogen receptor-independent breast cancer cells (MDA-MB-231). Indeed, taurine decreased the expression levels of matrix metalloproteinase 9 (MMP-9) and VEGF which are crucial proteins for the degradation of the ECM (85) and angiogenesis (51) (Fig. 1). In that sense, taurine compromised metastasis in both breast cancer cell lines, independently of the presence of estrogen receptor. Besides, it should be noted that estrogen exerted its significant effect on taurine uptake, through increased expression of TauT transporter in MCF-7 cells (86). It has been reported that $\mathrm{Na}^{+}$-dependent uptake of taurine through TauT transporter was activated by $17 \beta$-estradiol and p53 transcription factor, as shown by experiments in MCF-7 cells (87). As a result, taurine appears to be an attractive therapeutic agent because it can slow down the metastasis of breast cancer at aggressive stages, independent of the presence of estrogen.

Apart from the anti-oxidant and anti-angiogenic effect of taurine, it has been shown that taurine displays a strong chemopreventive effect on breast cancer (88). In support of this, taurine has been proved to alleviate methotrexate (MTX)-induced oxidative injury, by modulating immune response $(89,90)$ and by attenuating toxic side effects on renal cells, due to TAM administration in breast cancer cells $(89,90)$. The results were consistent with data derived from a clinical setting where cancer patients have shown alterations in plasma amino acids including taurine relative to their matched controls $(91,92)$.

In colon cancer, the main anti-tumor mechanism of taurine is based on upregulating apoptosis at both the transcriptional and translational levels $(42,58)$. For example, Zhang et al (42) have supported that taurine induced the transcription and translation of the PUMA gene in HT-29 colorectal cancer (CRC) cells. Focusing on the molecular mechanisms of taurine in more depth, taurine suppressed $\mathrm{p} 53^{-/-}$tumor cells more efficiently than $\mathrm{p} 53^{+/+}$tumor cells, indicating that the apoptosis-stimulatory action of taurine is the consequence of not only mitochondrial apoptotic pathway but also of multiple signaling pathways in colon cancer cells. In support, Liu et al (43) have shown that the mammalian sterile 20-like kinase 1-c-Jun N-terminal kinase (MST1-JNK) signaling pathway was essential for taurine-induced apoptosis in colon cancer cells (Caco-2 and SW620 cells). Following the treatment of colon cancer cells with taurine, the JNK signaling cascade was activated, either by transmitting direct signals to the MST1 target gene or by controlling the action of MST1 target via a feedback mechanism, with the ultimate aim of inducing apoptosis (43). Importantly, the growth-inhibitory effect of taurine was also proved either in colitis-model induced by TNBS (16) or in another colitis-inducible model caused by DSS (24). In particular, taurine appeared to alleviate clinical symptoms of colitis through its inhibitory action on diarrhea/bleeding, normalizing colon length, restoring histopathological alterations, and compromising the activity of myeloperoxidase (MPO) (24). In addition, the beneficial effect of taurine was ascertained in conditions where the MPO enzyme was absent. In that direction, it was shown that taurine protected human intestinal epithelial Caco-2 cells (MPO deficient) from oxidative damage, after their coculture with human macrophage-like THP-1 cells (93). Paradoxically, those research findings were incompatible with clinical data that supported the increment of taurine in colon cancer patients compared to healthy patients (94).

In prostate cancer, taurine has come to the forefront of research through its interference with the metastasis of tumor cells. Taurine seems to reduce the migratory potential of androgen-dependent human prostate cancer cells, though targeting matrix metalloproteinases (MMPs), which are considered crucial enzymes for the degradation of ECM. For example, the increased invasion of androgen-sensitive human prostate adenocarcinoma $\mathrm{LNCaP}$ cells and of androgendependent human prostate adenocarcinoma PC-3 cells was attenuated at $48 \mathrm{~h}$ and $8 \mathrm{~h}$, following treatment of cells with taurine (53). In particular, it has been shown that taurine (125-1,000 $\mu \mathrm{M})$ reduced the values of MMP-9 and stimulated the expression of epithelial markers such as E-cadherin and tight junction components, in a dose-dependent manner in prostate cancer cells (53). Notably, the increased expression of epithelial markers was accompanied by a marked reduction of mesenchymal genes such as N-cadherin, twist family BHLH transcription factor 1 (TWIST1), zinc finger E-box-binding homeobox 1 (ZEB1), SNAIL, and vimentin in LNCaP cells in response to taurine treatment (53). In this way, taurine was proved to be a promising therapeutic tool, restricting not only the migratory properties of androgen-dependent human prostate cancer cells but also reducing the recurrence of cancer with stem-like characteristics, thereby circumventing the possibility of tumor chemoresistance (95).

Besides, the proliferation of androgen-dependent human prostate cancer cells (PC-3 cells) has been supported to be hindered through the action of taurine haloamines (either $\mathrm{N}$-arachidonoyl taurine or $\mathrm{N}$-oleoyl taurine), that arise through their conjugation with fatty acids, thereby raising the possibility of using taurine haloamines as favorable therapeutic agents (54). Notably, there are two signaling pathways, that account for the distribution of $\mathrm{N}$-acyl taurines in human prostate adenocarcinoma. In particular, fatty acid amide hydrolase (FAAH) mediates the hydrolysis of $\mathrm{N}$-acyl taurines ( $\mathrm{N}$-arachidonoyl taurine, $\mathrm{N}$-oleoyl taurine) which are subjected to further catabolism (96). The FAAH has been shown to hydrolyze both $\mathrm{N}$-arachidonoyl taurine and $\mathrm{N}$-oleoyl taurine (97). Interestingly, the silencing of the FAAH enzyme can culminate in the concentration of $\mathrm{N}$-acyl taurines in the liver, kidney, and the central nervous system, reaching micromolar levels (98).

Likewise, taurine has been suggested as a diagnostic marker in bladder cancer, given that taurine levels were elevated in the endometrial wall of bladder cancer patients (56). In the urine of bladder cancer patients, the concentration of taurine seemed to be significantly elevated, as its value was below the sensitivity limit of $400 \mathrm{MHz}$ in control cases (56). Additionally to the functional significance of taurine in the diagnosis of bladder cancer, it has been suggested that taurine was effective in forestalling the proliferation of cervical cancer (CC) SiHa cells, through induction of apoptosis. The underlying molecular mechanism of taurine was based on upregulation of MST1 signaling pathway signaling pathway, leading to increased p53 nuclear transcriptional translocation (55). 


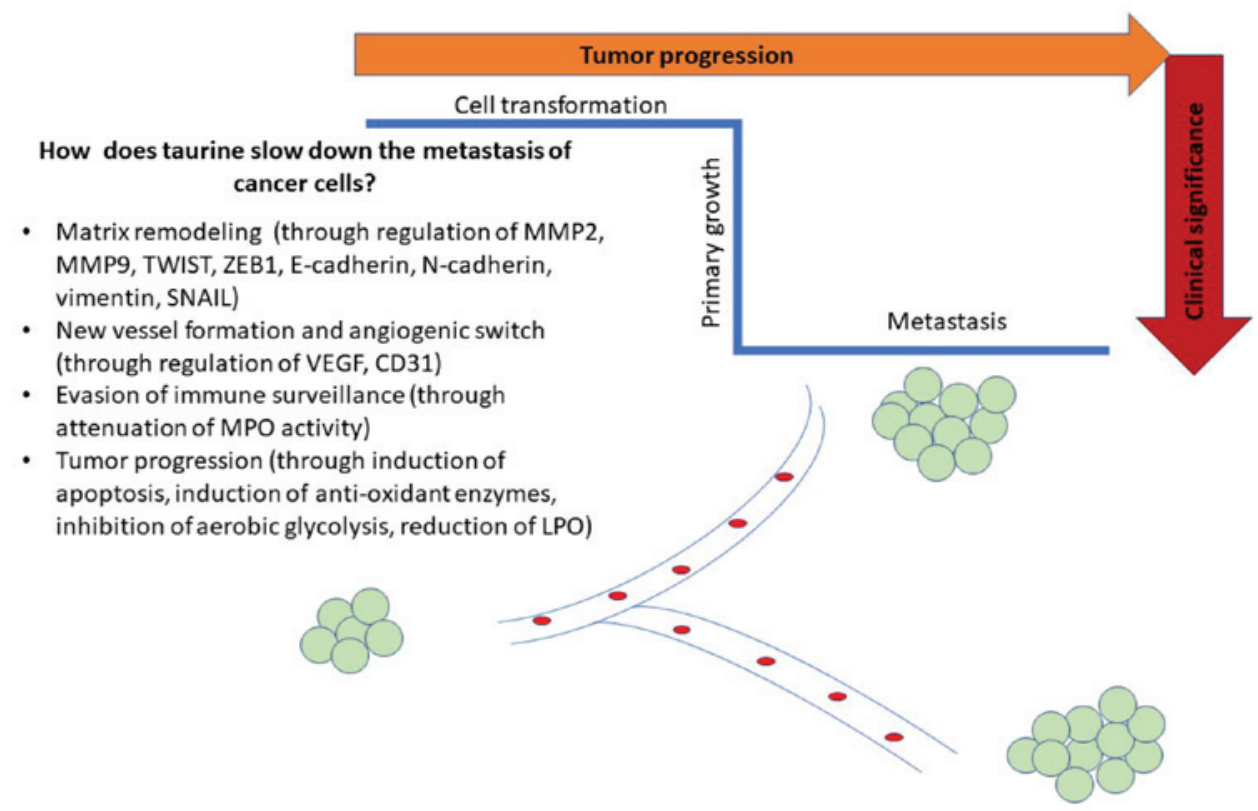

Figure 1. Schematic illustration of possible mechanisms of taurine towards attenuating metastatic procedure of cancer cells. Possible mechanisms include regulation of oxidant/anti-oxidant responses, decreasing the levels of angiogenic markers and causing matrix remodeling.

Furthermore, taurine has conferred protection against liver injury through its anti-oxidant properties (99). Several examples have demonstrated that taurine ameliorates the cytotoxicity mediated by various chemical compounds such as hydrazine, 1,4-naphthoquinone, and carbon tetrachloride (100) and by xenobiotics (101-103). In the case of arsenic-induced cytotoxicity, taurine has been shown to protect damaged hepatocytes, mainly by quenching free radicals and by detoxifying toxic metabolites (104). Taurine has also been illustrated to exert its cytoprotective effect against liver injury, either by interfering with LPO/protein oxidation or by reducing the accumulated hydrogen peroxide $\left(\mathrm{H}_{2} \mathrm{O}_{2}\right)$ /hydroxyl radicals $(\bullet \mathrm{OH})$ or by binding to ferrum $\left(\mathrm{Fe}^{2+}\right)$ like a chelator (105). Apart from its anti-oxidant activity, taurine has been illustrated to fortify hepatocytes against damage, by preventing osmolytic disturbance through ion overloading in the mitochondrial matrix (15).

Since taurine exerts beneficial effect on acute liver injury, it is plausible that taurine might be effective in abnormal cases of chronic liver injury such as hepatocarcinoma. Chronic liver injury exerts selective pressure on specific targets in the microenvironment, driving the neoplastic transformation of hepatic cells (106). Hepatocellular cancer (HCC) development can be stimulated in an inducible manner by diethylnitrosamine (DEN). The molecular mechanism of DEN is based on triggering irreversible hepatocellular necrosis coupled with compensatory proliferation (107). The DEN-mediated hepatic damage becomes apparent through increased oxidative stress in hepatocytes and it is followed by radical-based hepatic metabolic disturbance (108). It is important to refer to cytochrome P450 system (CYPs), especially CYP2E1 (109) that bio transforms DEN carcinogen to the enhanced generation of ROS $(107,108)$, by causing structural alterations through the formation of alkylated DNA adducts in hepatocytes (107). In this way, there is an aberrant regulation of redox homeostasis and stress adaptation in hepatocytes after DEN administration. In this context, taurine has been proved to help inhibit oxidative stress-related hepatic injury in DEN-treated rats. In particular, a single dose of taurine was shown to reverse the action of DEN carcinogen $(200 \mathrm{mg} / \mathrm{kg})$, by ameliorating the oxidative stress related-hepatic injury in mice $(92,109)$ and rats $(108)$. In molecular setting, taurine appeared to protect rat hepatocytes from DEN challenge, by normalizing the values of disturbed enzymes such as serum alanine aminotransferase (ALT), aspartate aminotransferase (AST), lactate dehydrogenase (LDH), $\gamma$ glutamyl transferase (GGT) activities (110) or by interfering with LPO (111). The main underlying mechanism of taurine was based on reducing oxidant responses including malondialdehyde (MDA), protein carbonyl (PC), and nitrotyrosine levels. However, the activity of anti-oxidant enzymes such as glutathione (GSH), glutathione peroxidase (GPx) levels, superoxide dismutase (SOD), and GSH transferase (GST) remained unchanged in DEN-challenged rats following taurine administration (110). Similarly, the anti-oxidant nature of taurine was confirmed in other settings of oxidative damage mediated liver injury. For instance, subcutaneous administration of taurine $(2.5 \% \mathrm{w} / \mathrm{w})$ was proved to improve histopathological findings within the time-window of 2 months in the liver of rats that had previously been subjected to subcutaneous injection of galactose $300 \mathrm{mg} / \mathrm{kg}$ for 5 days per week (112). Taurine ameliorated serum ALT, AST activities without any effects on the anti-oxidant responses such as SOD, GPx in rats harboring galactose-induced liver damage (112). Another example supporting the advantageous effect of taurine was observed in ethanol-induced hepatic dysfunction, due to its anti-oxidant nature, whereas rats treated with $\beta$-alanine (taurine transporter inhibitor) presented high susceptibility to ethanol-mediated liver damage (113).

Apart from its anti-oxidant activity, taurine taurine has been suggested as a promising effective agent against liver injury, by ameliorating membrane disintegration (114), inflammation (115), and calcium distribution (116). An interesting example supporting the anti-inflammatory effect 
of taurine was observed in the model of LPS-induced liver injury. Taurine conferred protection to hepatic cells from LPS-induced liver injury due to its anti-inflammatory nature, by reducing the secretion of pro-inflammatory mediators (including TNF- $\alpha$ and IL-6) and by elevating anti-oxidant responses [heme oxygenase-1 (OH-1), SOD] (115). In another case, taurine was documented to suppress the progression of alcoholic liver disease (ALD) in male Wistar rats, by preventing the transmission of signals through the LPS signaling pathway, in turn preventing the possible activation of Kupffer cells. In particular, the administration of taurine was reported to downregulate TNF- $\alpha$, ILs (IL-1 $\beta$, IL-6), lipopolysaccharide-binding protein (LBP), cluster of differentiation 14 (CD14), and nuclear factor- $\kappa \mathrm{B}(\mathrm{NF}-\kappa \mathrm{B})(117)$. The underlying mechanism of action of taurine was based on blocking the LPS-induced increase of calcium $\left(\mathrm{Ca}^{2+}\right)$ in Kupffer cells, taking into consideration that intracellular calcium $\left(\mathrm{Ca}^{2+}\right)$ plays an important role in LPS-stimulated cytokine production, during activation of Kupffer cells (116). In line with above, taurine hindered the phagocytosis mediated by Kupffer cells and reduced eicosanoid/TNF- $\alpha$ formation (118), thereby providing cytoprotection against damaged hepatocytes, due to its inhibitory action on the infiltration of immune cells in the liver and due to its osmoregulatory properties.

Hence, researchers have examined the possible synergistic effect of taurine with curcumin in vitro as well as in vivo conditions and they have supported that a treatment scheme consisting of taurine combined with curcumin could boost immune cell populations, culminating the therapeutic efficacy of curcumin in hepatocarcinoma. In particular, that combination treatment scheme was proposed to activate $\mathrm{CD} 4^{+} \mathrm{T}$-helper cells and to recruit $\mathrm{CD}^{+} \mathrm{T}$-cytotoxic cells in cultured human hepatoma (Huh-7) cells (119). Also, the treatment scheme of taurine combined with curcumin was able to eliminate potential malignant changes and to normalize IL-2, interferon- $\gamma$ (IFN- $\gamma$ ), $\alpha$-fetoprotein (AFP) and $\alpha$-L-fucosidase (AFU) levels in DEN-stimulated model of hepatocarcinogenesis (120).

In addition, taurine has been reported to inhibit the proliferation of murine melanoma B16F10 cells through the mitochondrial apoptotic pathway (121). The therapeutic effect of taurine was also shown in melanoma (B16F10 cells), through its anti-oxidant properties $(47,121)$. Interestingly, the beneficial effect of taurine was proved to be more pronounced in metastatic melanoma, which is usually treated with IL-2 immunotherapy, through the clonal expansion of lymphocytes (122). Besides, this type of IL-2 immunotherapy is effective against melanoma, but its response rates are hampered by vascular leak and lymphopenia (122). When taurine was conjugated to IL-2, taurine increased the efficiency of immunotherapy in a B16 melanoma pulmonary metastases model, by mitigating toxic side-effects of IL-2 itself (122). Interestingly, taurine exerted its protective mode on reducing the tumor burden and attenuated IL-2 toxic symptoms such as vascular leak syndrome and lymphopenia, in a model of metastatic melanoma (122). The results enabled the dose-escalation use of taurine, extending treatment scheme without causing any clinical sign of autoimmunity (122). In that sense, taurine maximized the anti-tumor effect of IL-2 immunotherapy in an in vivo metastatic melanoma model (123). The results became more understandable since IL-2 is importantly involved in activated-induced cell death (AICD). Notably, $\mathrm{T}$ cells are accumulated in order to defense tumor cells and they are eliminated due to AICD, under the rules of self tolerance (124). Additional research findings proved that the mechanism underlying the cytoprotective mode of taurine was attributed to the partial down-regulation of FasL-mediated apoptotic pathway in IL-2 sensitized Jurkat $\mathrm{T}$ cells, but not freshly isolated $\mathrm{T}$ cells through interference with $N F-\kappa B$ transcriptional activation (6). Accordingly, it is important to be noted that taurolidine (a taurine derivative) has been reported to prevent the possibility of disease relapse in mice bearing B16F10 melanoma cells, that were assigned to two different types of surgery (laparotomy or laparoscopy). The implementation of taurolidine gained significant traction due to its effect on recovering natural killer (NK) and lymphokine-activated killer (LAK) cell function, enhancing the functional properties of immune cells. As a result, taurolidine abrogated the effects of surgical trauma on primary and metastatic tumor growth without any interference with host anti-tumor surveillance mechanism, suggesting its potential significance in the management of tumor-bearing patients undergoing resection (124).

Furthermore, many skin tumors have shown increased susceptibility to glucocorticoids (GC), inducing robust responses but eventually acquired resistance and relapsed. According to the above viewpoints, Logotheti et al indicated that N-Bromotaurine ( $\mathrm{TauBr}$ ) might be suggested as a new therapeutic agent in the treatment of skin cancer cells that were GC unresponsive due to GC receptor (GR) impairment (125). It was proved that the therapeutic efficacy of $\mathrm{TauBr}$ arose through its synergism with cisplatin, exerting a growth-inhibitory effect on GC-resistant cells and thereby pointing out its GC-mimicking therapeutic effect (125). The results strengthened the potential therapeutic use of TauBr in other epithelial cancer types. Accordingly, taurine haloamines have exerted their action, showing good efficacy, tolerance, and insignificant toxic effects on patients who were refractory to conventional GC-based anti-inflammatory therapies $(4,126)$.

Taken together, there are several indicative clues on the therapeutic effect of taurine but there is diversity among results derived from individual cell lines of various malignancies. There is a lack of a comprehensive and comparative view across several cell lines of different malignancies. Further studies are needed to address this challenge and to shed light on the actual anti-cancer action of taurine.

Taurine attenuates the drug-mediated side effects. It is well-established that the administration of adjuvant chemotherapeutic drugs can lead to 5-year survival rates up to $70 \%$ for patients with non-metastatic disease. Even though this success is accomplished through supplementation of specific chemotherapeutic drugs, various combinations of approved chemotherapeutic agents (i.e., DOX, cisplatin, etoposide and ifosfamide) do not further increase patients survival over 10 years $(127,128)$. The mechanism underlying the efficacy of prescribed chemotherapeutic agents is non-specific, thus offering a window of off-target toxicity $(127,128)$. The function of multiple chemotherapeutic agents is related to multiple common unbearable complications including cardiotoxicity, 


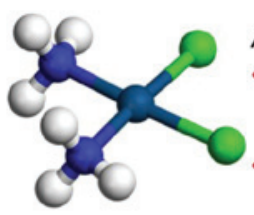

Attenuation of renal, neural injuries

- Anti-apoptotic, anti-oxidant action (low LPO, low iNOS activity)

Protection from DNA damage (increased 8-OHdG expression)

Cisplatin

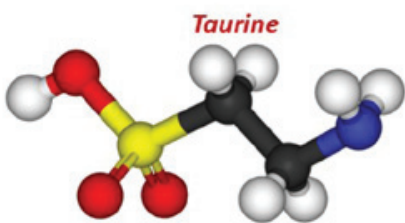

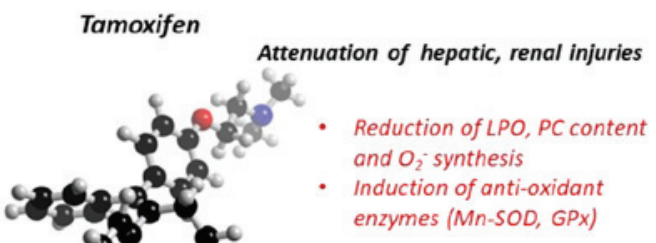

enzymes (Mn-SOD, GPX)

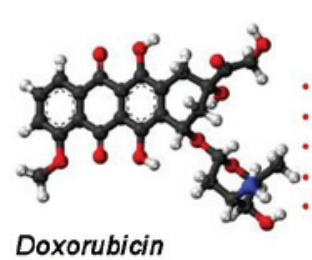

Doxorubicin
Attenuation of cardiac, hepatic, renal and testicular injuries

Regulation of JNK, p38 MAPK and PI3K/Akt pathways Induction of $\mathrm{Na}^{+}-\mathrm{K}^{*} / \mathrm{Ca}^{2+}$ ATPase

Stimulation of anti-oxidant enzymes (SOD, CAT, GST, GPX)

Reduction of GSSG, MDA, ROS content

Attenuation of inflammation through reduction of NF-KB,

COX-2, iNOS, pro-inflammatory cytokines (TNFa, II-6/16)

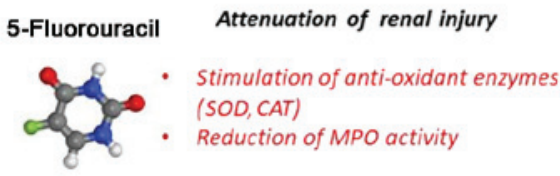

(SOD, CAT)

Reduction of MPO activity

Figure 2. Schematic illustration of possible mechansims of taurine towards attenuating toxic side-effects of chemotherapeutic drugs. Possible mechanisms include regulation of oxidant/anti-oxidant responses, targeting signaling pathways, decreasing the levels of pro-inflammatory cytokines and modulating DNA damage response.

nephrotoxicity, hearing loss, and the development of secondary malignancies $(127,128)$. It should be highlighted that toxicity also poses a significant challenge to the successful combination of existing therapeutic options, by exploiting the therapeutic index exerted by individual molecularly targeted drugs. Considerable attention should be directed to the extent of overlapping emerging toxicities derived from a possible combination therapeutic scheme, to maximize the therapeutic efficacy elicited by distinct drugs.

Beyond chemotherapy-related toxicity, the cancer recurrence is a topic of huge interest given that there are no available drugs that can overcome the resistance mechanisms of classic chemotherapeutic drugs. In this frame, a couple of studies have suggested that taurine is regarded as a promising agent to alleviate side-effects of several chemotherapeutic drugs and to ameliorate therapeutic outcomes, bypassing some challenges of drug resistance. Reinforcing this suggestion, the rational combination scheme of taurine with either chemotherapeutic drug has seemed to optimize the efficacy of existing standard treatment, by improving patient outcome and minimizing resistance conferred by the standard therapy. The appropriate time of the combination scheme is important to afford benefit to patient treatment. The benefits of combined drugs become apparent, exerting their action against different signaling pathways, their culminated efficacy and their reduced toxicity profiles. To select the appropriate combination of taurine with other agents, we should take into consideration that some cancer types display drug resistance due to redox disturbance, i.e., the disequilibrium between ROS and redox-sensitive survival proteins.

In 1992, it was reported that taurine content was eradicated after chemotherapy (71), whereas the expression levels of its precursor molecules remained constant (71). It has been highlighted that the pleiotropic nature of taurine enables the increased absorption of chemotherapeutic drugs (129), the alleviation of stress-induced insults (130), the reduction of radiation-induced injury (131) and the attenuation of inflammatory injury (132). Indeed, taurine holds great promise in some oxidative stress conditions mediated by ammonia (133) or acetaminophen (134) or gentamicin (135), without displaying any adverse effect. In addition, taurine represents an invaluable tool to deal with drug-induced myelosuppression or immunosuppression, which accounts for reducing the efficiency of either chemotherapeutic agent and for increasing the possibility of infections in patients with immunocompromised system (136). Considering the potential of taurine to alleviate the oxidative or inflammatory injury caused by other drugs, it is plausible that taurine enhances the tumor-inhibiting ability of chemotherapeutic agents (14), without off-target toxicity. In support of this notion, it has been proposed that taurine is capable of minimizing the injury triggered by classic chemotherapeutic drugs such as as DOX (137), 5-fluorouracil (5-FU) (76), TAM (138), and cisplatin (139) (Fig. 2). DOX is one of the most widely used anthracycline drugs against leukemia and sarcoma. DOX is regarded as a very effective chemotherapeutic drug (78), due to its capacity to prevent the replication of cancer cells as a topoisomerase II inhibitor $(140,141)$. As DOX forestalls topoisomerase II action, it forms a high oxidative and pro-inflammatory environment, thus causing DNA damage in cancer cells $(140,142)$. Following DOX administration, the DNA damage becomes apparent through DNA intercalation, which is evidenced through bonds between nitrogen bases of the complementary strands, thus causing disturbed DNA replication and transcription (143). Despite the beneficial impact of DOX against cancer, its use in the therapeutic arena is impeded due to its toxic side effects including cardiomyopathic failure and nephrotoxicity in patients (144-146).

Towards addressing the challenge of DOX mediated toxicity (either nephrotoxicity or hepatotoxicity or 
cardiotoxicity), researchers have proposed that taurine exerts synergistic therapeutic effect with DOX to culminate its therapeutic efficacy without off-target toxicity. Indeed, DOX-induced cardiac and testicular injuries were attenuated owing to the protection conferred by the anti-oxidant potential of taurine (78). The protective effect of taurine was also analyzed against DOX-induced testicular oxidative complications (77). In particular, 8-week old male rats were treated with either DOX alone or taurine alone or taurine plus DOX within 28 days and it was shown that taurine abrogated the DOX-induced testicular side-effects, by reducing oxidative stress (reduced GSH, increased GSSG and elevated MDA levels), by increasing activity of antioxidant enzymes including SOD, catalase (CAT), glutathione S-transferase (GST), GPx as well as membrane-bound enzymes such as $\mathrm{Na}^{+}-\mathrm{K}^{+}$and $\mathrm{Ca}^{2+}$ ATPases (77). The ameliorative effect of taurine against DOX-induced testicular abnormalities relied on activation of c-Jun N-terminal kinase (JNK), p38 mitogen-activated protein kinase (MAPK) pathways, and p53 transcription factor (77). Similarly, taurine was documented to provide marked protection against DOX-induced testicular damage, due to its anti-apoptotic role (78). Furthermore, the concurrent treatment scheme composed of DOX and taurine appeared to be effective in neutralizing cytotoxicity in murine melanoma B16F10 cells, mainly through the taurine's ROS scavengering capacity (143). The protective effect of taurine against DOX-induced cytotoxicity was attributed to cell cycle regulation and reduction of ROS production (143). Paradoxically, taurine employed its anti-oxidant nature to bypass the barriers posed by DOX-induced oxidant environment, thereby leading to DOX-mediated hepatocarcinoma cells to apoptosis (147). It was highlighted that taurine afforded protection against DOX-induced hepatoxicity through elevating SOD activity and GSH content in the livers of DOX intoxicated rats (147).

Taurine also acted as a renoprotective agent against DOX-induced acute kidney injury (AKI), by inhibiting apoptosis and inflammation. The ameliorative effect of taurine was evidenced against renal-induced oxidative injury of eight-week-old male Balb/c mice, that had previously been challenged with the DOX (15 mg/kg body weight) for $24 \mathrm{~h}$ and then subjected to taurine treatment $(50 \mathrm{mg} / \mathrm{kg}$ or $100 \mathrm{mg} / \mathrm{kg}$ body weight) for 5 days (148). In particular, taurine downregulated the renal expression of apoptosis-related proteins (p53, phospho-p53, caspase-9, and caspase-3) and renal expression of inflammation-related mRNAs such as nuclear factor- $\kappa \mathrm{B}(\mathrm{NF} \kappa \mathrm{B})$, cyclo-oxygenase $2(\mathrm{COX}-2)$, and inducible nitric oxide synthase (iNOS) (148). In another study, taurine reduced the expression levels of pro-inflammatory cytokines (TNF- $\alpha$, IL-1 $\beta$, IL-6, and COX-2) (149), that accumulated in renal tissues of DOX-challenged animal models, proving its anti-inflammatory action (150).

When taurine was administered to DOX-intoxicated rats, taurine directed cardiac cells to defend against DOX-related oxidative damage, thereby recovering them from the cell death pathways. The protective mode of taurine against DOX-induced cardiac oxidative stress was under the control of distinct signaling cascades (78). In particular, taurine ameliorated DOX-mediated injury, via the inhibition of the p53 transcription factor, JNK, MAPK dependent pathway and via the upregulation of phosphoinositide 3-kinase/protein kinase $\mathrm{B}$ (PI3K/Akt) pathway in cardiac cells (78).

Another chemotherapeutic strategy is 5-FU, which is directed to gastrointestinal solid tumors. The underlying mechanism of 5-FU is based on causing oxidative injury, as shown by elevated creatinine, blood urea nitrogen, and MDA content. However, the protective effect of 5-FU is limited due to severe toxic effects, including cardiac, renal, hepatic, diarrhea, myelosuppression, dermatitis, and reproductive system anomalies that arise (151-156). The action of 5-FU is non-specific, exerting its action not only in cancer cells but also in normal healthy cells, thereby leading to genomic instability and the accumulation of different toxic metabolites. When 5-FU is absorbed in renal cells, nephrotoxicity emerges due to reduced activity of either CAT or SOD and because of increased apoptosis (157). In this context, it has been shown that taurine alleviated FU-mediated side effects and in turn, increased 5 -FU therapeutic efficacy $(58,76)$. The ameliorative effect of taurine on FU-mediated complications became obvious through counteracting FU-induced histological changes such as distortion of normal cellular architecture, infiltration of inflammatory cells, and loss of cellular integrity (76). The underlying mechanism of taurine was based on reversing the increased MPO activity to eradicate FU-mediated abnormalities (76). Similarly, taurine proved to be effective in reversing sulfasalazine-mediated effects, owing to its anti-oxidant nature. In particular, taurine was presented as a recommended option against Crohn's disease, through its inhibitory action on LPO, and GSH status in both hepatic and renal cells (158).

TAM is another therapeutic option against various cancer types. The beneficial effects of TAM have shown to be hampered by side effects that arise in the liver (73), kidney (72), and breast (159), thereby preventing its clinical efficacy. Apoptosis, overproduction of toxic metabolites as well as elevated LPO are the main routes by which TAM displays its toxicity (160). Some studies have reported that taurine exerted protective action in vivo against TAM-induced hepatotoxicity (73) or nephrotoxicity (72). Taurine appeared to be effective in reducing $\mathrm{LPO}, \mathrm{PC}$ content, and $\mathrm{O}_{2}^{-}$synthesis, thereby ensuring normal redox homeostasis and maintaining the integrity of hepatic cells in TAM-treated animal models (73). Taurine seemed to be indispensable in restoring mitochondrial electron transport chain function in mouse liver mitochondria of TAM-treated animals, either by its ROS-scavenging capacity or by increasing activities of anti-oxidant molecules such as mitochondrial manganese-dependent SOD (Mn-SOD) and GPx (74), taking into consideration that taurine itself was devoid of apparent mitochondrial toxicity (74). As a result, taurine afforded protection to hepatic cells of TAM-treated animal models, either by reversing the decline of antioxidants or by the direct free radical-scavenging activity of taurine (74). Besides, it is important to mention that taurine proved to abrogate TAM-induced mitochondrial oxidative damage, mainly through its anti-oxidant action (138) and its potential to induce apoptosis in hepatic stellate cells (161).

In parallel, taurine appeared to confer protection to cells from the toxic effects caused by the concurrent administration of MTX and TAM. When MTX $(10 \mathrm{mg} / \mathrm{kg})$ and TAM $(50 \mathrm{mg} / \mathrm{kg})$ were intraperitoneally administered in Swiss albino mice, after the pretreatment of mice with taurine $(100 \mathrm{mg} / \mathrm{kg})$ 
for nine days, it was proved that pretreatment of mice with taurine seemed to attenuate genotoxicity, through the synergism of two chemotherapeutic drugs (162). The underlying mechanism of taurine was based on increasing the reduced GSH content and hindering chromosomal aberrations in both somatic and germ cells. In that sense, it was proposed that taurine provided therapeutic effectiveness not only alleviating toxic side effects but also preventing the incidence of tumor recurrence following chemotherapy (162).

Cisplatin (CDDP) is another classical chemotherapeutic agent that is commonly prescribed in treatment for a wide range of solid tumors including testicular and cervical carcinoma, because of its efficacy and low cost $(163,164)$. However, its clinical effectiveness is hindered due to its toxic side effects in hepatic and renal cells (165). Interestingly, it has been reported that cisplatin accounts for renal dysfunction in a significant proportion of cancer patients (25-35\%) (166). Following cisplatin administration, the patients developed apparent tubular injury at the proximal tubular level due to the induction of inflammation, oxidative stress, apoptosis, and hypoxia (167-169). The main mechanisms of cisplatin-mediated nephrotoxicity were based on increasing ROS formation, DNA oxidation, and TNF- $\alpha$ secretion through increased $\mathrm{NF}-\kappa \mathrm{B}$ transactivation (170). An interesting example was shown in ovarian cancer women with advanced disease who acquired resistance to cisplatin and relapsed, as shown by their shorter disease-free intervals (171). In that frame, taurine seemed to inhibit ovarian cancer cell proliferation, by enhancing the therapeutic efficacy of cisplatin and by alleviating cisplatin-mediated side effects $(172,173)$. The induction of mitochondrial apoptotic cell death was the main underlying mechanism by which taurine exerted its advantageous action in cisplatin-treated human CC (174). In another study, the beneficial effect of taurine was demonstrated to be based on ameliorating oxidative DNA damage signals, through inhibition of p53 nuclear transcriptional translocation and elevation of anti-oxidant responses, thereby culminating in the therapeutic efficacy of cisplatin (175). However, it is worth mentioning that the cisplatin resistance of CC A2780 cells, was manifested through osmotic disequilibrium due to an increased taurine uptake from cells (176).

Additionally, researchers have provided deep insight into the ways by which taurine mediated its protective action against nitrative stress that is usually encountered as renal injury in cisplatin-treated animal models (177). In one interesting case, a single intraperitoneal injection of cisplatin $(15 \mathrm{mg} / \mathrm{kg}$, or $25 \mathrm{mg} / \mathrm{kg}$ ) in male Wistar rats deteriorated kidney function for 7 days and taurine $(5 \% \mathrm{w} / \mathrm{v})$ was administered in drinking water of rats four days before the injection of cisplatin (175). The precise mechanism underlying the cisplatin-mediated nephrotoxicity was the oxidative stress and taurine protected renal cells against cisplatin-induced nephrotoxicity, through its anti-inflammatory capacity, its potential to boost anti-oxidant responses, its anti-apoptotic action and its ability to relieve from DNA damage insults such as 8-hydroxy-2-deoxyguanosine (8-OHdG) expression (175). Following treatment with taurine, the expression levels of citrulline, iNOS, and 8-nitroguanidine were decreased in cisplatin administered animal models (139). Besides, it is important to note that taurine transporter function was proved to be dysfunctional in disturbed renal conditions mediated by cisplatin (172). In that sense, the favorable effect of taurine against cisplatin-induced acute nephrotoxicity was illustrated to be consistent with the deficiency of taurine transporter (TauT) in renal cells, following administration of cisplatin (178).

The toxic effects of cisplatin are not only directed to renal cells but also expand to neural cells. To prove the advantageous effect of taurine against cisplatin-induced neural injury, researchers intraperitoneally injected $10 \mathrm{mg} / \mathrm{kg}$ of cisplatin in rats for 13 days and they observed various histological changes including a marked decrease in the total traveled distance, average speed, total mobile time, total mobile episode, number of crossing and absolute turn angle, leading to neurological defects (179). The administration of 100 or $200 \mathrm{mg} / \mathrm{kg}$ taurine for 13 consecutive days before cisplatin injection was reported to be amazingly effective in improving neurological abnormalities of rats (179). Taurine treatment caused a marked improvement in brain anti-oxidant status, which became apparent through elevated acetylcholinesterase activity, decreased oxidative stress indices [low nitric oxide (NO), and LPO levels], increased survival of neural cells in the cerebral cortices, and in the hippocampus (179). Moreover, taurine eliminated the dendritic arborization and mean diameter of the somata of pyramidal neurons in the cisplatin treated rats, implying that taurine afforded protection against cisplatin-induced neurotoxicity (179).

Additionally, it has been reported that the challenge of either cisplatin or paclitaxel (PTX) chemoresistance in both ovarian cancer cells (A2780 and OAW42) was bypassed through the action of taurine which impeded cancer stem cell population. Taurine treatment is a powerful way to enable ovarian cancer cells to respond to the therapeutic efficacy of classic chemotherapeutic drugs (180). Also, nuclear magnetic resonance (NMR) spectroscopy supported that the long term administration of metformin accounted for the upregulation of taurine in ovarian cancer cells, that had previously displayed strong insensitivity to either cisplatin or PTX. Therefore, taurine was considered as the underlying factor that inhibited cancer stem cell population.

Ifosfamide is a chemotherapeutic agent, which can lead to proximal renal tubular injury that mimics Fanconi syndrome. Fanconi syndrome is considered a disease of the proximal renal tubules of the kidney in which glucose, amino acids, uric acid, phosphate, and bicarbonate are passed into the urine instead of being reabsorbed. The study by Badary (181) highlighted that ifosfamide injections in animal models rendered them to display all the characteristics of Fanconi syndrome such as wasting of glucose, electrolytes, and organic acids, along with increased serum creatinine and urea, and diminished the creatinine clearance rate. Taurine markedly attenuated some signs of renal dysfunction induced by ifosfamide, through various mechanisms: diminished creatinine, urea and albumin serum levels due to elevated creatinine clearance rate and a marked decline in total and fractional excretion of $\mathrm{Na}^{+}, \mathrm{K}^{+}, \mathrm{PO}^{-3}$ and glucose (181). However, taurine did not alter the efficacy of ifosfamide in mice with Ehrlich-Lettre ascites carcinoma (EAC) cells (182).

In the meantime, the ani-neoplastic effect of taurine has arisen in great interest, due to its capacity to orchestrate the inflammatory milieu. It is well established that 
chemotherapeutic drugs exert their effect not only on cancer cells but also on the strongly proliferated bone marrow hematopoietic cells $(183,184)$. Taurine has been presented as a promising agent to circumvent chemotherapy-induced side effects, due to its known immune-regulatory properties. Some researchers believe that taurine is an effective immune adjuvant, which can play a role in chemotherapy drugs, and has multi-directional advantages (185). For example, the beneficial action of taurine has been proved to be helpful in attenuating the side effects of chemotherapy, thus potentiating the immune function of mouse T-cell lymphoma. After quantification of pro-inflammatory mediators IL-4, IL-12 and IFN- $\gamma$, it was observed that there was a greater decline in the taurine/chemotherapy-treated group of mice compared to the chemotherapy group (186). In addition, taurine emerged as the promising agent that bypassed the toxic injuries derived from the combination of gemcitabine and cisplatin, thereby maximizing the efficacy of chemotherapeutic drugs (187). The therapeutic efficacy of taurine was presented very strongly against peripheral T-cell lymphoma, given that the tumor inhibition rate was remarkably higher in the group treated with chemotherapy drugs and taurine compared to chemotherapy group alone (187). Taurine exerted its ameliorative action, by normalizing Th1/Th2 cytokine levels in both spleen and thymus (186).

Of note, the Lewis lung carcinoma-bearing mice presented accelerated tumor regression following taurine treatment $(40,80$, and $160 \mathrm{mg} / \mathrm{kg})$ combined with cyclophosphamide, compared to the chemotherapeutic group alone. Interestingly, all the doses of taurine treatment increased the classic parameters of the immune system (lymphocytes, macrophages, neutrophils), as demonstrated by elevated bone marrow nucleated cells, augmented white blood cells, increased spleen index as well as elevated thymus index (14). Alleviation of myelosuppression and elevation of the phagocytic activity of peritoneal macrophages were the main mechanisms behind the immunoregulatory role of taurine against cyclophosphamide-induced damage. In that sense, taurine reinforced cellular immune function and attenuated the immunosuppression of cyclophosphamide (14). Accordingly, recent findings proved that taurine up-regulated $\mathrm{T}$ cell responses in the thymus of immunosuppressive mice, that had previously been injected with dexamethasone (Dex) for 7 days. In particular, long-term taurine supplementation (at a dose of $200 \mathrm{mg} / \mathrm{kg}$ for 30 days) was presented to be remarkably effective in the development of $\mathrm{T}$ lymphocyte subpopulations. Interestingly, taurine significantly increased the number of $\mathrm{CD}^{-}{ }^{-} \mathrm{CD} 8^{-}$double-negative (DN), $\mathrm{CD} 4^{+} \mathrm{CD}^{+}$ double-positive (DP), $\mathrm{CD}^{+}$single-positive $\left(\mathrm{CD}^{+}\right)$and $\mathrm{CD}^{+}$ $\mathrm{SP}\left(\mathrm{CD}^{+}\right)$cells in Dex-treated mice compared with the control group. Furthermore, the $\mathrm{CD} 4^{+} / \mathrm{CD}^{+}$cell ratio did not display any difference between thymus of Dex-induced immunosuppressive mice, without or with the administration of taurine (136). From a clinical perspective, it was highlighted that taurine attenuated the immune-suppressing adverse effects of cyclophosphamide therapy by boosting the phagocytic capacity of macrophage and neutrophil cells to dampen inflammatory responses (14). Similarly, young adults with acute lymphoblastic leukemia (ALL) were characterized by lower incidence of febrile episodes, neutropenia, and infectious complications following taurine treatment compared to the placebo group that had received one of the classic chemotherapeutic strategies (188). During taurine treatment, the numbers of leukocyte populations were elevated, explaining why the overall episodes were lower in ALL patients (188). In that way, taurine exhibits immune-regulatory properties to ameliorate the unbearable complications present in ALL. In the same context, it was reported that chemotherapy mediated adverse effects (nausea, vomiting) were attenuated through taurine supplementation in patients bearing ALL and receiving one chemotherapeutic scheme (70).

\section{Formation of taurine haloamines}

In the regions of inflammatory or infected tissues, neutrophils are recruited and they are regarded as the first-line defense by eradicating the invading microorganisms through the production of either oxidants or microbicidal proteins (189-191). When neutrophils engulf invading microbes, superoxide anion $\left(\mathrm{O}_{2}^{-}\right)$formation is increased at the expense of adenosine triphosphate (ATP) synthesis due to the action of nicotinamide adenine dinucleotide phosphate (NADPH) oxidase. This occurs since the dysfunctional respiratory chain accumulates electron donors, thus leading the transfer of electrons from NADPH oxidase to oxygen, leading to an oxidative burst. Then, $\mathrm{O}_{2}^{-}$undergo a dismutation reaction, converting them to accumulated $\mathrm{H}_{2} \mathrm{O}_{2}$. In activated neutrophils, MPO enzyme uses $\mathrm{H}_{2} \mathrm{O}_{2}$ to react with halides (chloride $/ \mathrm{Cl}^{-}$or bromide/ $\mathrm{Br}$ ), producing hypohalous acids $(\mathrm{HOCl}$ or $\mathrm{HOBr})$ which are very toxic oxidants, impairing cell homeostasis $(190,192,193)$. It is important to mention that hypochlorous $(\mathrm{HOCl})$ and hypobromous $(\mathrm{HOBr})$ acids are highly reactive but unstable oxidants with strong microbicidal and cytotoxic activities $(5,194)$.

In activated neutrophils, taurine fulfills its cytoprotective and antioxidant properties through the reaction of taurine with $\mathrm{HOBr}$ or $\mathrm{HOCl}$, contributing to the formation of taurine haloamines including $\mathrm{N}$-Chlorotaurine $(\mathrm{TauCl})$ or $\mathrm{N}$-Bromotaurine (TauBr), respectively (4). It is important to note that hypohalous acids arise from the neutrophil-myeloperoxidase (MPO) or eosinophil peroxidase (EPO) halide system of metabolism during inflammation $(193,195)$. In this way, taurine serves its primary role to protect neutrophils from their self-destruction caused by the hypohalous acid-mediated oxidative injury under inflammatory conditions (4). Also, taurine protects the surrounding cells from the inflammatory and oxidative damage, through the generation of taurine haloamines.

It is commonly accepted that taurine haloamines are long-lived oxidants that are less toxic than hypohalous acids and confer protection against oxidative stress in inflammatory sites. Due to the antimicrobial and antiseptic properties of taurine haloamines, they seem to be invaluable in the treatment of local mucosal and skin infections (196). Between taurine haloamines, TauBr has stronger microbicidal activity and is more potent membrane-permeable than $\mathrm{TauCl}$ (197). In contrast, $\mathrm{TauCl}$ is thought to be more stable than TauBr, explaining its use as a local curative agent in a wide spectrum of infections (126). Interestingly, $\mathrm{TauCl}$ is considered to be a charged molecule, with low permeability 
capacity that renders impossible the inactivation of the highly sensitive thiol enzyme glyceraldehyde-3-phosphate dehydrogenase (GAPDH) (198-200).

It should be highlighted that taurine haloamines are only produced in $\mathrm{O}_{2}^{-}$-generating neutrophils. Interestingly, neutrophils derived from chronic granulomatous disease (CGD) patients are unable to produce $\mathrm{TauCl}$, because they cannot genetically produce $\mathrm{O}_{2}^{-}$(201). The $\mathrm{O}_{2}^{-}$producing neutrophils are equipped with NADPH oxidase to ensure the formation of taurine haloamines in conditions of oxidative burst (201). In this sense, taurine haloamines serve as important modulators of the immune system, down-regulating pro-inflammatory cytokine production, ensuring the compromised immune response. that influences the synthesis of cytokines. However, taurine haloamines are not used in clinical practice, because of their rapid degradation in blood. The beneficial effects of taurine haloamines will be leveraged only if the barrier will be circumvented. Therefore, stable TauBr compounds such as $\mathrm{N}$-monobromo-2,2-dimethyltaurine (Br-612), N-dibromo-2,2-dimethyltaurine (Br-422) and Bromamine T (BAT) were devised in an attempt to identify the anti-microbial and anti-inflammatory properties of taurine analogs that were stable (202).

\section{Anti-microbial properties of taurine haloamines}

The loss of virulence and lag of bacterial regrowth has been ascribed to the oxidizing effect of either $\mathrm{TauCl}$ or $\mathrm{TauBr}$, providing 'chlorine covers' or 'bromine covers' (creating either covalent $\mathrm{N}-\mathrm{Cl}$ or $\mathrm{N}-\mathrm{Br}$ bonds) on the surface of target proteins $(203,204)$. When either $\mathrm{TauCl}$ or $\mathrm{TauBr}$ is introduced into the cytosol, the chlorination or bromination is followed and the oxidation of intracellular proteins is necessary for complete eradication of pathogens (126). In particular, either $\mathrm{TauCl}$ or $\mathrm{TauBr}$ has been shown to exert its action, mediating the chlorine or the bromine transfer to the amino groups on proteins of microbial membranes without the involvement of catalysts, suggesting that the lone pair of electrons on the nitrogen atom of amino groups of bacterial proteins associates with the chlorine atom of $\mathrm{TauCl}$ or with the bromine atom of $\mathrm{TauBr}$ as an electrophilic chemical reaction. It is important to note that the extent of chlorine transfer reaction elicited by $\mathrm{TauCl}$ or the extent of bromine movement mediated by $\mathrm{TauBr}$ depends on the type of microorganism species, incubation time, $\mathrm{pH}$, and the temperature (204).

In response to microbial and parasite infections, neutrophils and eosinophils secrete abundant amounts of $\mathrm{TauCl}$ and $\mathrm{TauBr}$. Taurine haloamines are considered strong microbicidal agents, eradicating a wide variety of Gram-positive or Gram-negative bacteria, fungi, viruses, and protozoa (4), while taurine haloamines do not exert any cytotoxicity to host tissues (4). For example, $\mathrm{TauCl}$ and $\mathrm{TauBr}$ have been shown to kill the schistosomula of Schistosoma mansoni (205). Schistosomula can be eradicated by $1 \mathrm{mM}$ TauCl or by $100 \mu \mathrm{M}$ TauBr to nearly $40 \%$ (205).

As a general note, TauBr is an effective therapeutic agent against chronic sinusitis, otitis media, acne vulgaris, and periodontal diseases $(126,203,206-212)$. Interestingly, TauBr killed a specific type of skin bacteria (Propionibacterium acnes) and it was used as a classic therapeutic agent in patients who developed resistance to standard-of-care treatment (clindamycin). In the clinical setting, the majority of patients displayed remission of acne vulgaris symptoms at a rate of $65 \%$ after long-term treatment with TauBr, without side effects $(207,213)$. TauBr is supposed to exert greater microbicidal effect than $\mathrm{TauCl}$ at very low concentrations $(<10 \mu \mathrm{M})$ and neutral $\mathrm{pH}$.

Even though TauCl was initially considered as a molecule without bactericidal capabilities, $\mathrm{TauCl}$ is a potent bactericidal compound, downregulating the extravagant bactericidal potential that could be detrimental to the host. In this sense, taurine chlorination confers the advantage of compromising $\mathrm{HOCl}$-induced tissue damage while sustaining anti-microbial properties. The bactericidal potential of $\mathrm{TauCl}$ is ascribed to the oxidation of sulfhydryl groups which are in the bacterial cell membrane. TauCl exerts its action, neutralizing both gram-positive (Staphylococcus aureus and Staphylococcus epidermidis) and gram-negative bacteria (Escherichia coli, Pseudomonas aeruginosa, and Proteus mirabilis) when it was administered at the following concentration range (12.5-50 $\mu \mathrm{M})$ (209). It has also been shown that taurine imparted its preventive action against Candida spp., Aspergillus spp., Fusarium moniliforme and Polytrichum commune (126), and inactivate viruses including type 1 and 2 human herpes simplex virus (HSV), adenovirus, human immunodeficiency virus (HIV)-1, and influenza viruses (126). Interestingly, the killing capacity of $\mathrm{TauCl}$ against Shiga toxin of enterohemorrhagic E. coli, its molecular mechanism relied on oxidizing the thiols and aromatic amino acids of the bacterial proteins (214). Accordingly, the lipophilic nature of $\mathrm{NH}_{2} \mathrm{Cl}$ was incorporated into the hydrophobic bacterial cell membranes, achieving phagocytosis of E. coli (190).

\section{Anti-inflammatory and anti-oxidant properties of taurine haloamines}

At inflammatory sites, toxic hypohalous acids are neutralized by taurine, generating taurine haloamines ( $\mathrm{TauCl}$ or $\mathrm{TauBr}$ ) (215). TauCl and $\mathrm{TauBr}$ are products of either MPO or EPO halide system and they serve as modulators of the immune system (215). Following the activation of neutrophils or eosinophils, the release of taurine haloamines is accelerated to confer important protection to many nearby cells in several respects from inflammatory injury (216) and to attenuate oxidative stress $(13,217-219)$. Initially, taurine haloamines have been identified to confer protection to neutrophils from toxic hypohalous acids (hypochlorous or hypobromous), which are detoxified with the presence of taurine (4). Secondly, taurine haloamines have been shown to exert strong microbicidal properties, neutralizing either bacteria or fungi or viruses (126). Thirdly, taurine haloamines have been illustrated to display strong anti-inflammatory properties that are primarily related to the reduction of various pro-inflammatory mediators such as TNF- $\alpha$, ILs (IL-1 $\beta$, IL-6, IL-8, IL-12), NO, prostaglandin $\mathrm{E}_{2}\left(\mathrm{PGE}_{2}\right)$ and chemokines in both rodent and human leukocytes (197,220-223). In particular, TauCl was demonstrated to exert a strong anti-inflammatory activity in many cell types $(5,212,224)$ whereas $\mathrm{TauBr}$ was proved to suppress the synthesis of pro-inflammatory cytokines (TNF- $\alpha$, IL-6, IL-10, IL-12p40) and NO in macrophages $(220,225)$. 
In that sense, taurine haloamines inhibited inflammatory cell trafficking at injured sites and probably blocked the incidence of chronic inflammation (4). Importantly, it was proposed that the anti-inflammatory action of haloamines relied on the induction of heme-oxygenase-1 (HO-1) in a dose-dependent manner (215,225-227). The aforementioned results were evaluated since $\mathrm{HO}-1$ exerts a potent anti-oxidant and anti-inflammatory action through degradation of heme to bilirubin, free iron, and carbon monoxide (CO) (228). When HO-1 enzyme is upregulated, $\mathrm{CO}$ production is elevated, subsequently enabling cells to be functional and safe against oxidative injury caused by overproduction of $\mathrm{O}_{2}{ }^{-}$and $\mathrm{NO}$, though inhibition of either NADPH oxidase or iNOS enzyme (229). Taurine haloamines have been shown to play a crucial role in averting the conversion of acute into chronic inflammation, thus impairing the possibility of chronic inflammatory diseases. Taurine haloamines have been reported to protect cells from inflammation-derived oxidative stress, through elimination of toxic $\bullet \mathrm{OH}$ and additional $\mathrm{ROS}$ formation, thereby reducing the cytochrome catalyzed electron transfer to oxygen and ensuring cellular homeostasis. Besides, it is important to note that the anti-oxidant potential of taurine haloamines has been highlighted to be accomplished in three different ways. One possible mechanism was manifested through the conjugation reaction of taurine with mitochondrial tRNA. In particular, Schaffer et al (13), and Jong et al (230) supported that taurine inhibited $\mathrm{O}_{2}^{-}$generation and is required for normal respiratory chain activity as well as the appropriate synthesis of ATP through the formation of mitochondrial taurine-conjugated tRNAs. Alternatively, taurine haloamines appeared to reverse the redox inequilibrium, by increasing the expression of many antioxidant enzymes, such as HO-1, SOD, and GPx, peroxyredoxin-1 (Prx-1), thioredoxin-1 (Trx-1), and CAT (4).

In inflammatory-associated conditions, the therapeutic potential of taurine haloamines has been highlighted in both in vitro and in vivo settings. The research group of Marcinkiewicz has provided convincing evidence that taurine haloamines blocked the synthesis of COX-derived eicosanoid such as $\mathrm{PGE}_{2}$ in LPS/IFN- $\gamma$ stimulated macrophages (LPS/IFN- $\gamma$ J774A.2 mfs) via enhancing HO-1 enzyme expression without altering COX-2 expression. Besides, the inhibitory action of taurine haloamines against $\mathrm{PGE}_{2}$ accumulation was confirmed in HO-1 deficient environment (227). In contract, taurine did not exert any significant impact on $\mathrm{PGE}_{2}$ levels in stimulated macrophages (227). One potential underlying hypothesis was that taurine haloamines induced HO-1 enzyme at inflammatory sites to confer protection to neighboring non-activated cells against oxidative stress (227). The beneficial impact of taurine haloamines was also shown in vivo, using DSS-induced experimental colitis model. The colon cancer regression was observed after the reaction of exogenously administered taurine with endogenous hypohalous acids at inflammatory sites (24). The anti-inflammatory capacity of taurine haloamines was probably based on their capacity to hinder phagocyte function and impair respiratory burst (24).

In rheumatoid arthritis (RA), taurine haloamines have been shown to inhibit the protein expression of IL-6 and PGE $_{2}$ with similar potency. Even though both taurine haloamines are considered powerful regulators of inflammation, $\mathrm{TauCl}$ has been shown to exert more predominant anti-inflammatory effects compared to those elicited by TauBr. In particular, TauCl inhibited IL-8 and VEGF synthesis secreted by fibroblast-like cells (FLS) from patients with RA whereas TauBr did not affect the levels of IL- 8 and VEGF. Besides, neither agent exerted a great impact on regulating NO generation and iNOS protein expression (221).

The anti-inflammatory capacity of $\mathrm{TauCl}$ has been highlighted in all activated types of leukocytes in vitro $(231,232)$ and animal models of both acute and chronic inflammatory diseases (233-235). In 1996, Quinn et al (236) supported that TauCl suppressed $\mathrm{PGE}_{2}$ expression, mediating post-translational effects on COX-2 mRNA in RAW 264.7 macrophages exposed to LPS or IFN- $\gamma$. Then, the anti-inflammatory activity of $\mathrm{TauCl}$ was proved in macrophages in response to an inflammatory stimulus. Importantly, it was documented that the anti-inflammatory ability of TauCl was tightly linked to increased HO-1 activity in macrophages (LPS/IFN- $\gamma$ J774A.2 mfs), suggesting that $\mathrm{TauCl}$ was a strong inducer of HO-1, without any effects on COX-2 protein expression (227). Regarding the molecular mechanisms involved, TauCl used different ways to tame inflammation by targeting gene expression of proinflammatory cytokines, cell adhesion molecules, and pro-inflammatory mediators such as COX-2 or iNOS in a cell type-dependent manner (224). In particular, other research findings supported that $\mathrm{TauCl}$ hampered the synthesis of pro-inflammatory mediators such as NO, TNF- $\alpha$, ILs (IL-6/8), PGs in RAW 264.7 macrophages of murine origin in exposure to LPS or IFN- $\gamma$, illustrating its important regulatory effect on macrophage function (237-240). In these cases, the suppression of pro-inflammatory genes was consistent with inhibition of NO production in stimulated macrophages following TauCl treatment $(237,240)$. Notably, the anti-oxidant activity of $\mathrm{TauCl}$ (a detoxified form of $\mathrm{HOCl}$ ) was ascribed to its preventive action against the catalytic activity of iNOS directly by targeting the enzyme rather than by interfering with the interaction of cofactors with iNOS (240). Many antioxidant proteins including $\mathrm{OH}-1, \mathrm{Gpx}$, Prx 1 and CAT were reported to be increased, upon exposure of macrophages to $\mathrm{TauCl}$ (241). Similarly, it was reported that $\mathrm{TauCl}$ reduced the expression of $\mathrm{O}_{2}^{-}$, ILs (IL-6/8) in human polymorphonuclear leukocytes $(223,242)$. In another study, TauCl interfered with indoleamine-2,3 dioxygenase (IDO) activation, contributing to low expression levels of IFN- $\gamma$ (243). As a result, the anti-inflammatory properties of $\mathrm{TauCl}$ were reported to be tightly associated with the inhibition of many pro-inflammatory mediators, such as $\mathrm{O}_{2}^{-}$, NO, TNF- $\alpha$, IL- $1 \beta,-2,-6,-8$, and $-10, \mathrm{PGE}_{2}$, macrophage inflammatory protein-2 (MIP-2), monocyte chemoattractant protein-1 and -2 (MCP-1/2), and MMPs (4).

Regarding the underlying molecular mechanism of $\mathrm{TauCl}$ in more depth, TauCl appeared to coordinate the synthesis of pro-inflammatory mediators through the regulation of $\mathrm{NF}-\kappa \mathrm{B}$ transcriptional transactivation $(239,244,245)$. Beyond identifying NF- $\mathrm{KB}$ as the master transcription factor, the landscape remained obscure as to which signaling pathways were activated to regulate the activation of the NF- $\kappa \mathrm{B}$ transcription factor, in various cell types under inflammatory conditions following $\mathrm{TauCl}$ stimulation. The research pertinent to the 
anti-inflammatory action of $\mathrm{TauCl}$ was focused on the regulation of MAPK, which are composed of JNK, p38, and extracellular signal-regulated kinase (ERK), accounting for the activation of the NF- $\kappa \mathrm{B}$ transcription factor $(246,247)$. On one side, it was mentioned that $\mathrm{TauCl}$ at $1 \mathrm{mM}$ (not taurine) suppressed LPS-mediated NO production in a dose-dependent manner, inhibiting ERK phosphorylation and retaining p38 activity in RAW 264.7 macrophages. Elucidating the inhibitory effect of $\mathrm{TauCl}$ on the ERK signaling pathway, researchers proved that the inhibition of Ras activation was the main principle of TauCl activity (248). The attenuation of LPS-induced inflammation relied on the downregulation of ERK and its downstream NF- $\mathrm{B}$ activation, considering that inhibition of Ras small GTPase was the most profound cause behind the anti-inflammatory action of $\mathrm{TauCl}$, without affecting the activity of activator protein (AP)-1 (248). Nonetheless, ERK activation was not observed in resting RAW 264.7 macrophages after treatment with TauCl alone (248), but ERK signaling pathway was affected in human vein endothelial cells in response to $\mathrm{TauCl}$ (198). In Jurkat T cells, it was proposed that TauCl did not exert any effect on ERK phosphorylation (224). In the same frame, the capacity of TauCl to induce HO-1 was reported to be modulated only using p38 MAPK inhibitor (not ERK inhibitor) in J774.2 macrophages (227). Consistent with the above, it is plausible to consider that the effect of $\mathrm{TauCl}$ has been employed in a cell-type dependent manner since some reports support that both ERK and p38 are required for interfering LPS-mediated NO production $(249,250)$ and others have claimed that only p38 activation is linked to LPS-mediated NO synthesis (251).

After a thorough scrutinization of research reports, it was illustrated that $\mathrm{TauCl}$ of various concentrations hindered $\mathrm{NF}-\kappa \mathrm{B}$ activation in distinct cell types of myeloid or lymphocytic or mesenchymal origin $(224,239,244)$. NF- $\kappa \mathrm{B}$ activation was the main causative mechanism by which $\mathrm{TauCl}$ caused the decline of pro-inflammatory cytokines in both macrophages and leukocytes. TauCl seemed to impart its anti-inflammatory action, hindering the NF- $\kappa \mathrm{B}$ transcription that is a cornerstone for the synthesis of pro-inflammatory cytokines, either mediating oxidation of $\mathrm{I} \kappa \mathrm{B}-\alpha$ in methionine residue at position 45 (244) or decreasing phosphorylation of IkB- $\alpha$ in serine residue at position 32 (239). The oxidation of IkB- $\alpha$ at methionine 45 was the main mechanism of neutralizing $\mathrm{NF}-\kappa \mathrm{B}$ activation mediated by TauCl in Jurkat $\mathrm{T}$ cells activated by TNF- $\alpha$ (244). Conversely, the anti-inflammatory action of $\mathrm{TauCl}$ was evidenced through suppressing the IkB- $\alpha$ phosphorylation of serine 32 in the activated NR8383 macrophage cells stimulated by LPS and IFN- $\gamma$ (239). Similarly, TauCl appeared to inhibit IL-1 $\beta$-derived NF- $\kappa$ B DNA binding activity in fibroblast-like synoviocyte cells (FLS) derived from RA patients (252).

Apart from the effect of $\mathrm{TauCl}$ on innate immunity, many research reports have provided convincing evidence that $\mathrm{TauCl}$ had a strong anti-arthritic effect, as shown in various experimental animal models and samples isolated from RA patients $(233,253,254)$. Interestingly, TauCl seemed to be remarkably effective not only in macrophages but also in mesenchymal cells of inflammatory-associated disorders. For instance, $\mathrm{TauCl}$ was demonstrated to mediate its preventive action on pro-inflammatory mediators, by inhibiting the expression levels of TNF- $\alpha$, ILs (IL-6/8) in distinct adipose tissue samples of RA patients [articular adipose tissue (AAT), subcutaneous adipose tissue (ScAT)] as well as in samples of rats derived from adjuvant-induced arthritis $(253,255)$. There was also a marked reduction in the production of pro-inflammatory cytokines (IL-6, IL-8, and PGE 2 ) secreted by FLS, that originated from the joints of RA patients, following TauCl treatment $(252,256,257)$. Behind the mechanism underlying the ameliorative effect of $\mathrm{TauCl}$ against arthritis, Kontny et al (256) proved that $\mathrm{TauCl}$ was a specific and potent inhibitor of COX-2 protein expression in fibroblast like synoviocyte cells of rheumatoid arthritis patients (RA FLS) after IL $1 \beta$ stimulation. In that sense, it was illustrated that the cell viability of fibroblast like synoviocyte cells of RA patients was reduced, following concurrent treatment with platelet-derived growth factor (PDGF) and TauCl. The anti-proliferative effect of TauCl was also demonstrated in fibroblasts which had been stimulated with either basic fibroblast growth factor (bFGF) or TNF- $\alpha$. Following treatment with TauCl, the inhibition of FLS proliferation was attributed to the increased nuclear accumulation of the p53 transcription factor, causing the cell cycle arrest $(256,258)$. In addition, the attenuating effect of $\mathrm{TauCl}$ against impaired FLS function of RA patients was ascribed to reduced MMP synthesis $(226,259)$. As a result, TauCl could ameliorate RA-associated symptoms through its blocking effect on inflammatory injury.

It is important to note that there are controversial data on the impact of $\mathrm{TauCl}$ on collagen-induced arthritis (CIA) course. Some researchers have reported that TauCl hinders the development of CIA whereas others have proved that $\mathrm{TauCl}$ alleviates the severity of symptoms $(233,260)$. CIA can be applied to genetically susceptible (DBA $1 / \mathrm{J}$ ) mice after their immunization with native type II collagen in adjuvant, to delineate the pathogenesis and the signaling pathways involved in RA (4). On one hand, the onset of CIA has been illustrated to be slowed down in mice that had received $\mathrm{TauCl}$ before or after collagen injections, thereby reducing the possibilities for arthritis emergence in these mice (233). The development of arthritis was attenuated in the DBA1/J mice with CIA, following $\mathrm{TauCl}$ treatment, independently whether $\mathrm{TauCl}$ therapy was applied early (after primary immunization) or late (after booster immunization) during the CIA course (233). Focusing on the underlying mechanism of $\mathrm{TauCl}$, it has been substantiated that $\mathrm{TauCl}$ inhibited collagenase activity action, thereby delaying the incidence of CIA arthritis (261). On the other hand, systemic application of $\mathrm{TauCl}$ has proved to alleviate severe unbearable complications, that were presented in the DBA $1 / \mathrm{J}$ mice with CIA, such as paw swelling, arthritic scores, cartilage damage, synovial inflammation and bone erosion (260). In particular, TauCl mediated its action through interfering with lymphocyte proliferation and osteoclast formation, thereby leading to a strong remission of synovial inflammation, amelioration of cartilage damage and bone erosion through inhibition of $\mathrm{NF}-\kappa \mathrm{B}$ activation (260). Following TauCl treatment, the synthesis of pro-inflammatory mediators (TNF- $\alpha$, IL-1, and IL-6) was also reduced, thus compromising bone destruction (4). Similarly, the therapeutic benefits of $\mathrm{TauCl}$ have arisen in septic arthritis mediated by Staphylococcus aureus, when $\mathrm{TauCl}$ was locally administered after a single dose of Staphylococcus aureus (254). TauCl has 
been reported to exert its protective action by delaying the development of arthritis and ameliorating bone erosion and cartilage damage $(233,253,254)$. It should be noted that no advantageous effects of $\mathrm{TauCl}$ were observed when bacteria and $\mathrm{TauCl}$ were systemically administered (254).

In this regard, researchers have provided compelling evidence that $\mathrm{TauCl}$ suppressed inflammation-mediated bone destruction, through its capacity to interfere with osteoclast formation. To determine the mechanism which was involved in the inhibitory effect of $\mathrm{TauCl}$ on osteoclast formation, bones of mice with CIA, and the receptor activator of $N F-\kappa B$ ligand (RANKL)-stimulated bone marrow-derived monocyte/macrophage precursor cells (BMMs) were used. The results proved that the nuclear factor of activated $\mathrm{T}$ cells 1 (NFATc1) was the main osteoclast-specific transcription factor that was negatively affected by $\mathrm{TauCl}$. As a result, reduced tartrate-resistant acid phosphatase (TRAP), cathepsin $\mathrm{K}$ activity, calcitonin receptor, and the impaired formation of multi-nucleated osteoclasts were observed following treatment with $\mathrm{TauCl}$ (260). Those findings proposed that TauCl might be leveraged as a novel therapeutic strategy against bone diseases, which are characterized by excessive bone resorption.

Beyond the anti-arthritis mode of $\mathrm{TauCl}$, the beneficial effect of $\mathrm{TauCl}$ has become clear in mesenchymal cells of RA, ultimately altering arachidonic acid metabolism that is commonly associated with an inflammatory response. An interesting research report by Kim et al (226) provided insight into the favorable impact of $\mathrm{TauCl}$ on $\mathrm{RA}$, by using FLS isolated from RA patients following stimulation with adiponectin or IL-1 $\beta$, and their treatment with TauCl. In both modes of stimulation, it was proved that $\mathrm{TauCl}$ was a promising inhibitor of MMPs (226). In the case of adiponectin stimulation of synoviocytes, $\mathrm{TauCl}$ exerted a greater inhibitory effect than that mediated by IL-1 $\beta$ stimulation, as evidenced by higher nuclear shutting of NF- $\mathrm{BB}$ transcription factor (226).

Even though TauCl has been reported to hinder the proliferation of TNF- $\alpha$ stimulated neutrophils by inducing mitochondrial apoptosis (262), TauCl has been shown to confer protection to phagocytic cells from cell death caused by the overproduction of $\mathrm{O}_{2}^{-}$and $\mathrm{H}_{2} \mathrm{O}_{2}$ in an inflammatory milieu. Specifically, $\mathrm{TauCl}$ has been shown to reduce the proliferation in mitogen-stimulated human peripheral blood lymphocytes (223), in IL-3-dependent murine hematopoietic prolymphocytic B cells (263), in human skin fibroblasts (264) and tumor cells (B lymphoma osteosarcoma cell lines) $(265,266)$. Growth-inhibitory effect of TauCl was presented in distinct cell types in response to various stimuli, confirming that there are conserved mechanisms that warrant further investigation.

Thus, TauCl has emerged as a new therapeutic option, following spinal cord injury. The anti-inflammatory action of TauCl was linked to its anti-oxidant activity, as manifested by the reduction of ROS and other inflammatory mediators including TNF- $\alpha$ (267).

\section{Therapeutic perspectives and clinical studies}

Even though advances have been reported in the field of therapeutics, both cancer type-dependent drug responses and chemo-resistant therapies necessitate their enrichment with novel pharmaceutical agents. The role of pharmacology in medicine is to investigate new therapeutic drugs by using appropriate models to acquire a greater understanding of the molecular interactions that determine the outcome of cancer cells. Several in vitro and in vivo methods have been performed for the pre-clinical assessment of anti-inflammatory drugs such as taurolidine or $\mathrm{TauBr}$ or $\mathrm{TauCl}$ and some clinical case reports have been employed.

Initially, elimination of tissue damage has been attributed to the anti-inflammatory, not the anti-bacterial potential of taurine (4). So, researchers examined the therapeutic effect of taurolidine as well as $\mathrm{TauBr}$ and $\mathrm{TauCl}$, which arise through the reaction of taurine with hypohalous acids.

Taurolidine is a derivative of taurine and its common use is directed to deal with various infections. From a structural perspective, taurolidine is a bis-(1,1-dioxyperhydro-1,2,4-thiadiazinyl-4) methane, comprising of two taurolidine rings derived from taurine and three molecules of formaldehyde, by forming a two-ringed structure bridged by a methylene group (268). The first clinical report supported that taurolidine (taurine derivative) could be used in the prevention of severe surgical infections (sepsis, peritonitis and pancreatitis), given that taurolidine harbors strong bactericidal activity against antibiotic-resistant bacteria (269-271). Taurolidine exerts anti-endotoxin, anti-bacterial, and anti-adherence properties. Taurolidine is now included in a new catheter lock solution to hinder catheter-related infections $(272,273)$. It has been pointed out that taurolidine may have anti-bacterial action which is independent of the resultant taurine metabolites. The anti-bacterial activity of taurine has also been ascribed to its anti-inflammatory properties, as manifested by inhibition of IL-1 and TNF- $\alpha$ (274).

Interestingly, intravenous administration of $2 \%$ taurolidine was given to a gastric cancer patient with liver metastasis for 39 cycles, each of which lasted 7 days of treatment per month (300 mg/kg body weight per day). Taurolidine therapy was devoid of any toxicity and rendered cancer cells stable, without enabling them to colonize to other sites. Despite the encouraging results by taurolidine administration following chemotherapy, the gastric cancer patient died due to myocardial infarction (275). Similarly, two glioblastoma patients achieved tumor remission due to taurolidine application, within 4 months but then, they succumbed to the aggressiveness of tumor cells (276).

In order to use the beneficial effect of taurine in a clinical setting, certain obstacles should be addressed. Taurine is poorly absorbed in the gastrointestinal tract and so it is plausible that taurine is metabolically degraded in both the gut and the liver. Taurine is also characterized by unfavorable pharmacokinetics, a very strong hydrophilic nature, lipophobic character, and fast rate of extraction through urine, which potentially explains the difficulties to use taurine as a therapeutic agent. For this reason, further investigations prompted to understand the anti-inflammatory properties of taurine derivatives in a clinical setting.

Experiments have shown that $\mathrm{TauCl}$ exerts good tolerability in human tissues, as it is used in the treatment of infections of the eye, skin, outer ear canal, nasal and paranasal sinuses, and oral cavity. For example, the therapeutic efficacy of $\mathrm{TauCl}$ has been proven at phase II clinical studies for the treatment of external otitis, crural ulcerations, and keratoconjunctivitis $(126,210,277)$. 
Similarly, it has been proved that TauBr seems to be the most suitable topical agent for the treatment of biofilm-associated infections such as chronic sinusitis, otitis media, acne vulgaris, and periodontal diseases (4). The therapeutic efficacy of TauBr has especially been observed in the treatment of biofilm-associated infections (278). In another study, forty patients with mild to moderate inflammatory facial acne vulgaris were randomly enrolled and received either $\mathrm{TauBr}(3.5 \mathrm{mM})$ or classical antibiotic option (clindamycin-1\%) for 6 weeks, twice a day. The results were very encouraging concerning the promising therapeutic action of TauBr because the symptoms of patients with acne vulgaris were recovered in $80 \%$ of patients, with acne lesions being reduced from 65 to $68 \%$. Interestingly, $\mathrm{TauBr}$ appeared to ameliorate inflammatory acne vulgaris lesions even in patients with antibiotic resistance (207). In addition, multiple sclerosis (MS) patient with herpes zoster skin presented attenuation of clinical symptoms when $0.8 \%$ TauCl was administered for four days, followed by concurrent treatment with $0.8 \%$ $\mathrm{TauCl}$ and $1.0 \% \mathrm{TauBr}$ in the next three days. Interestingly, the treatment scheme composed of taurine haloamines was able to bypass the resistance developed in a MS patient to valacyclovir (279). Recently, it was shown that chronic multi-bacterial biofilm scalp infection was treated with the combined topical application of the active halogen compounds $\mathrm{TauCl}$, $\mathrm{TauBr}$ and BAT (280). This is the reason why taurine and taurine haloamines can be considered potential drugs in infectious and chronic inflammatory diseases. Certainly, the use of taurine haloamines as anti-inflammatory agents entails some risks that remain unknown.

In conclusion, both in vitro and in vivo studies as well as clinical trials will encourage us to consider taurine and taurine haloamines as potential drugs in human medicine, including infectious and chronic inflammatory disease. However, taurine and taurine haloamines warrant further investigation to examine their therapeutic efficacy in a clinical setting. Along with optimizing the drugs brought to the clinic, patient selection is a prerequisite for clinical trials. This refined patient matching can be achieved by our growing understanding of the interaction between signaling molecules involved in either inflammation or cancer. Undoubtedly, more studies are urgently needed to delineate mechanisms of action elicited by either agent as well as their pharmacodynamic and pharmacokinetic properties. Experimental models are need based on pharmacological principles to predict the intended therapeutic response of taurolidine, $\mathrm{TauBr}$ or $\mathrm{TauCl}$.

\section{Significance of IncRNA TUG1 IncRNA in cancer}

Long non-coding RNAs (lncRNAs) are outlined as noncoding transcripts with a length of more than 200 nucleotides, and they were originally discovered through the large-scale sequencing of mouse cDNA libraries (281). As a general note, lncRNAs deserve particular attention due to their involvement in many physiologic processes and their reported abnormal expression in pathologic circumstances, including cancer $(282,283)$. Since the impaired expression is tightly related to human malignant tumor formation (284), delineating how lncRNAs control gene expression is the major focus for cancer research (285). Interrogating the function of tumor-associated IncRNAs and elucidating their subsequent clinical impact comes with a surge of excitement. LncRNAs are important regulators in cancer progression through their participation in cancer proliferation, cancer invasion, replicative senescence, resistance to radiation and drugs, and reprogrammed energy metabolism (286,287). At molecular setting, tumor-associated lncRNAs can serve either (I) as decoys to direct transcription factors in a spatial-temporal manner (288) or (II) as carriers to transmit regulatory signals for transcription among cells (289) or (III) as scaffolds to aggregate a variety of RNA-associated proteins in transcriptional complexes (290) or (IV) as competitive endogenous RNAs to interact with functional microRNAs to cause their silencing (291) or (V) as guide molecules to recruit chromatin-modifying enzymes, conferring epigenetic regulation of target genes (292-294). LncRNAs are also critical for regulating cellular biological processes, through their binding to kinase proteins, thereby causing the respective conformational changes (295). Tumor-associated lncRNAs have revolutionized therapeutics in cancer research, enabling an outpour of studies documenting the startling contribution of lncRNAs to either cancer progression or remission.

In this context, taurine upregulated gene 1 (TUG1) is a lncRNA, that has been identified due to its upregulation during retinal development in response to taurine treatment (296). Accumulating evidence has supported the overexpression of lncRNA TUG1 in different disease contexts, including MS (297), diabetes mellitus (DM) (298), chronic kidney disease (CKD) (299), and chronic obstructive pulmonary disease (COPD) (300). In the diabetic nephropathy and CKD, one potential mechanism underlying the action of lncRNA TUG1 is based on increasing the transcription of peroxisome proliferator-activated receptor- $\gamma$ coactivator $1 \alpha$ (PGC-1), thus enabling the improved mitochondrial bioenergetics $(299,301)$. Similarly, lncRNA TUG1 has been illustrated to mediate its ameliorative effect on pancreatic insulin secretion and pancreatic islet dysfunction (302).

Besides, the 1ncRNA TUG1 has been regarded as an eminent lncRNA in cancer progression (303) among multiple cancer-associated lncRNAs $(304,305)$. Many research studies have provided a wealth of information on the significance of TUG1 lncRNA in many cancer types, dictating its contribution to the tumor progression as well as its prognostic value for unfavorable survival of cancer patients (Fig. 3). The lncRNA TUG1 is presented to be dysregulated in a variety of malignancies, indicating its significance in orchestrating tumor landscape. An apparent conundrum is that lncRNA TUG1 acts either in promoting cancer cell proliferation or abrogating cancer progression. In particular, the expression of multiple target genes can be controlled, either by activation or suppression, through the action of lncRNA TUG1 in a cell-type dependent manner, determining the end transcriptional result of target genes (306). From one perspective, accumulating evidence has proved that lncRNA TUG1 functions as an oncogene $(303,307)$, predicting poor prognosis for melanoma (308), bladder cancer (309), sarcoma (310), hepatocarcinoma (311), and colon cancer (312). For example, lncRNA TUG1 has been reported to be increased either in osteosarcoma due to its modulation of diverse transcription variants (310) or in hepatocellular carcinoma due to epigenetic suppression of Kruppel like factor 2 (KLF2) protein (311), implying the significant gene-regulatory activity of IncRNA TUG1 in cancer. Even 
though most of the studies have illustrated the overexpression of this lncRNA in cancer tissues as opposed to noncancerous counterparts, few studies have supported the opposite trend. For example, it has been reported that lncRNA exerts a tumor-suppressor role, being downregulated in glioma (313) and lung cancer [non-small cell lung cancer (NSCLC)] (314). It should be pointed out that the tumor-suppressor role of lncRNA TUG1 is consistent with the evidence supporting that promoter of lncRNA TUG1 contains conserved p53 binding sites (314).

At the molecular setting, aberrant signal transduction by lncRNA TUG1 has been mainly shown to be mediated through its interaction either with Polycomb repressive complex 2 (PRC2) or with miRNAs (315) (Fig. 4). There is convincing evidence supporting that the regulatory function of lncRNA TUG1 could be elicited, through its binding to PRC2 complex, thus reorganizing the transcriptional landscape in target genes through chromatin remodeling (316). When the lncRNA TUG1 functioned as a guide molecule to recruit the chromatin-modifying complex to target genes, subsequent epigenetic alterations such as changes in DNA methylation patterns of histone modifications were followed, thus abrogating target gene expression. For that purpose, researchers considered that the lncRNA TUG1 was the main determinant behind chromatin orientation and they tried to understand how lncRNA TUG1 spatially organized the chromatin, affecting the expression of target genes involved in tumorigenesis. Indeed, the association of lncRNA TUG1 with PRC2 complex caused the inhibition of specific genes through methyltransferase activity conferred by the PRC2 complex, as it has been observed in $20 \%$ of lncRNAs (317). Interestingly, it was proven that the lncRNA TUG1 interacted with enhancer of zeste homolog 2 (EZH2) enzyme, epigenetically causing the reduced expression levels of tumor suppressor genes, through trimethylation of histone 3 at lysine 27 (H3K27me3) in target genes (318), as shown in human NSCLC (319), gastric cancer (320) and hepatocellular carcinoma (321). For example, it was illustrated that overexpressed lncRNA TUG1 expression levels epigenetically modified homeobox B7 (HOXB7) expression, through its association with polycomb repressive complex 2 (PRC2), contributing to remission of NSCLC (319). Furthermore, Yang et al (322) showed that the association of lncRNA TUG1 with the PRC2 complex was crucial to coordinate the gene expression of transcriptional units in the three-dimensional space. In response to growth signals, it was pointed out that growth-regulatory genes could be shuttled between polycomb bodies and interchromatin granules, according to the recruitment of PRC2 chromatin modifying complex to lncRNA TUG1 (322).

On the contrary, numerous related studies have demonstrated that the regulatory network between IncRNA and microRNAs, determined the tumor progression either positively or negatively. Indeed, the lncRNA TUG1 exerted its action as a miRNA sponge consistent with the known function of lncRNAs as competing endogenous RNAs (ceRNAs), to antagonize the function of miRNAs (323). The classical 'sponge' function of lncRNAs is identified as the main mechanism accounting for posttranscriptional regulation of target genes. LncRNA binds to miRNA, competitively inhibiting the binding of the miRNA to its target mRNA, thus stimulating the expression of the downstream target mRNA (324). Moreover, miRNA functions as an inhibitor of target mRNA, counteracting target gene expression through its base-pairing with the 3-untranslated region (3-UTR) of target mRNA (325). In this direction, comprehensive detailed research progress has been performed regarding the inhibitory functions of lncRNA TUG1 against miRNAs. The lncRNA TUG1 was proved to be recruited at specific sites of following miRNAs: miR-212-3p, miR-132-3p, miR-145, miR-26a, miR-9, miR-34a-5p, miR-382, miR-300, miR-335-5p, miR-144, miR-138-5p, miR-219, miR-142, miR-153, miR-299, miR-600, and miR-129-5p, thus inhibiting the expression of aforementioned miRNAs and affecting cancer progression in a cancer type-dependent manner (326-330). In addition, many studies shed light on the involvement of lncRNA TUG1 in increased metastasis of distinct tumor types, either directly targeting mesenchymal genes or indirectly through its interaction with multiple miRNAs. Indeed, lncRNA TUG1 interacted with the following miRNAs (miR-144, miR-145, miR-26a, miR-9-5p, miR-34a-5p, miR-229, and miR-300), thus leading to radioresistance, carcinogenesis, invasion, angiogenesis, and blood-tumor barrier permeability (313,331-336). A characteristic example demonstrated that lncRNA TUG1 directed glioma stem cells to the uncontrolled growth, by impeding the degradation of stemness genes by retaining miR-145 and hindering the expression of neural differentiation-associated genes, upon Notch signaling (188). Another example showed that the IncRNA TUG1 accounted for the upregulation of mesenchymal markers (involving vimentin) and the downregulation of epithelial markers in $\mathrm{CC}$ (337). In that regard, the inhibitory action of lncRNA TUG1 against distinct miRNAs was shown to contribute to the exacerbation of cancer progression.

Apart from the contribution of 1 ncRNA TUG1 to cancer through either chromatin remodeling or sequestration of miRNAs, the IncRNA TUG1 has been reported to determine the outcome of various malignancies, through its involvement in various signaling cascades. For example, the lncRNA TUG1 exerted its regulatory action on either osteosarcoma or glioma or gallbladder carcinoma or oral squamous cell carcinoma through its potential to interfere with either PI3K/Akt or Notch or transforming growth factor- $\beta$ (TGF- $\beta$ ) or Wnt/ $\beta$-catenin $(331,338,339,340)$. Interestingly, it was proved that the therapeutic efficacy of cisplatin could be increased through overexpression of lncRNA TUG1 in breast cancer, relying on inhibiting the Wnt signaling pathway through regulation of miR-197/nemo like kinase (NLK) (341). In another case, lncRNA TUG1 gathered considerable attention as an oncogene in colon cancer, due to its capacity to enable the constitutive transmission of Wnt/ $\beta$-catenin signals (342). Similarly, it was proved that lncRNA TUG1 enabled the pancreatic cancer cells to acquire mesenchymal characteristics, by competitively inhibiting TGF- $\beta /$ Smad signaling pathway (343).

When introducing the contribution of the lncRNA TUG1 to lung cancer, many independent studies highlighted the downregulation of IncRNA TUG1 in the tissues derived from NSCLC patients compared to control samples. Interestingly, patients with a 2-year follow-up from lung cancer presented a marked decline of IncRNA TUG1 depending on the 


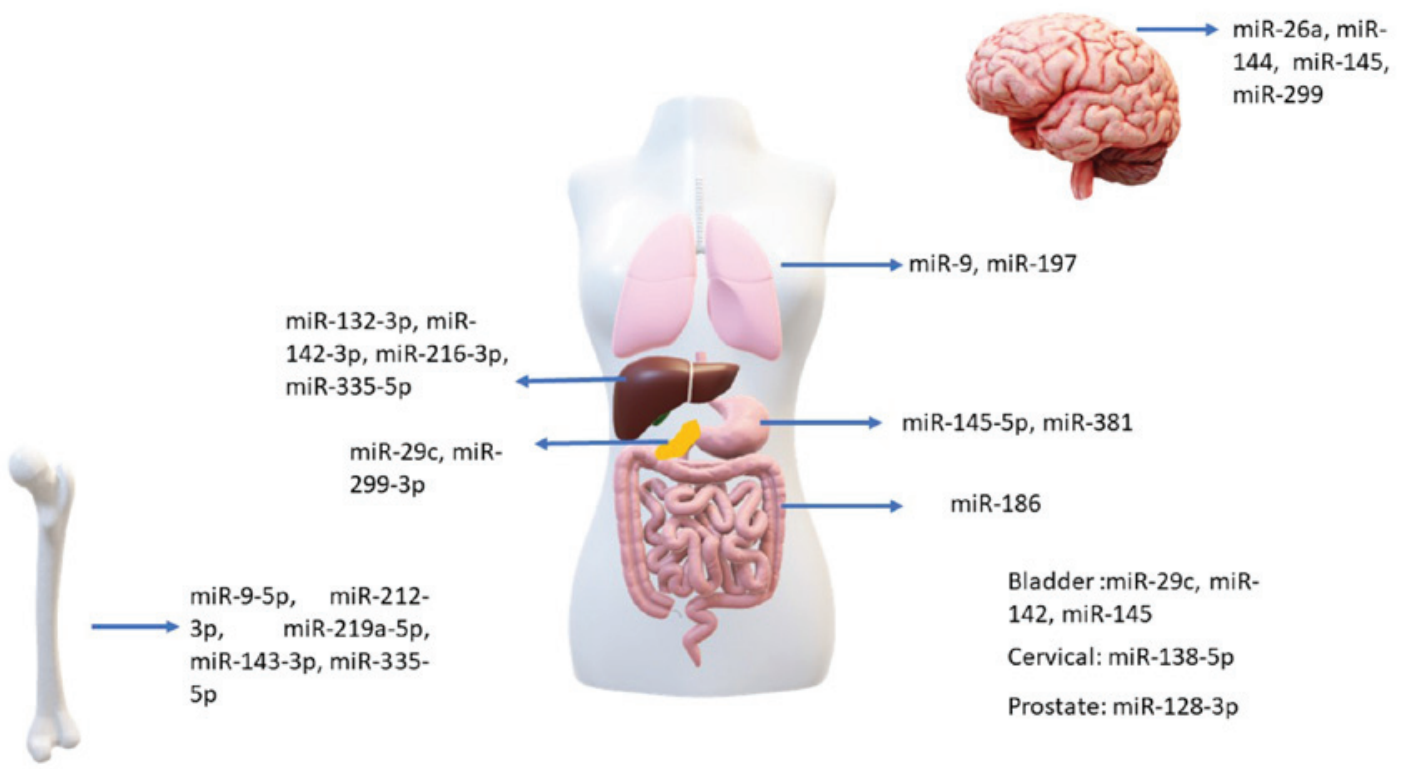

Figure 3. Schematic representation of the human body highlighting how lncNRA TUG1 exerts its sponging action against distinct miRNAs in a cell-type dependent manner.

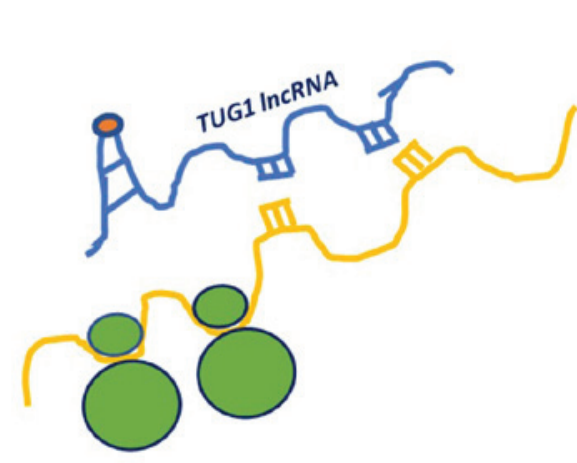

TUG1 IncRNA functions as oncogene, by sponging:

miR-9 (breast cancer), miR-9-5p (osteosarcoma), miR-29c (pancreatic cancer), miR-29c (bladder cancer), miR-128-3p (prostate cancer), miR132-3p (liver cancer), miR-138-5p (cervical cancer), miR-142 (bladder cancer), miR-142-3p (liver cancer), miR-143-3p (osteosarcoma), miR144 (BTB), miR-145 (glioma), miR-145 (gastric cancer), miR-145 (bladder cancer), miR-145 (cholangiocarcinoma) miR-148a-3p (esophageal squamous cell carcinoma) miR-212-3p (osteosarcoma), miR-216-3p (liver cancer), miR-219a-5p (osteosarcoma), miR-299 (glioma), miR-299-3p (pancreatic cancer), miR-335-5p (liver cancer), miR-335-5p (osteosarcoma), miR-381 (gastric cancer)

TUG1 IncRNA functions as a tumor suppressor gene, by sponging:miR26-a (glioma), miR-197 (breast cancer)

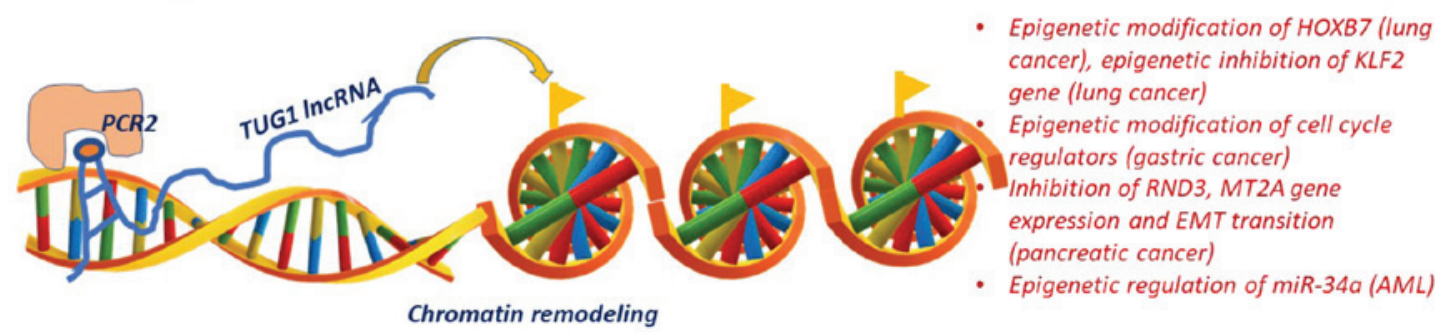

Figure 4. The lncRNA TUG1 regulate transcriptional and post-transcriptional processes important through its sponging action against miRNAs or through chromatin remodeling in a cancer type dependent manner. The inhibitory action of IncRNA TUG1 against miRNA-mediated mRNA degradation can exert either positive or negative impact on cancer progression. During chromatin remodeling, the lncRNA TUG1 usually recruits polycomb repressive complex 2 (PRC2) to chromatin sites that preclude RNAPII chromatin binding.

tumor-node-metastasis (TNM) staging, tumor size, and patient progression (344). The lower TUG1 expression was related to increased tumor burden and the poorer overall survival of patients, implying that lncRNA TUG1 can function as an independent predictor for overall survival of NSCLC patients. The expression pattern of lncRNA TUG1 was also confirmed in an independent cohort study of patients, indicating the CUGBP Elav-Like Family Member 1 (CELF1) as a possible target of lncRNA TUG1 (345). Interestingly, it was proved that the underlying molecular mechanisms of lncRNA TUG1 were based on inhibiting epigenetically homeobox B7 (HOXB7) expression, through interference with either AKT or MAPK signaling cascade (314). These data suggested that TUG1 is regarded as a potential tumor-suppressive lncRNA in NSCLC.

In gastric cancer, lncRNA TUG1 seemed to be overexpressed, in turn leading to metastasis of gastric cancer into lymph nodes (327). TCGA validated the overexpression of lncRNA TUG1, which predicts for poor prognosis of gastric cancer (346). In particular, the lncRNA TUG1 was regarded as an unfavorable predictor of gastric cancer, because patients diagnosed with gastric cancer displayed such increased expression patterns depending on the following clinicopathological features: the invasion depth of tumor, the tumor site and the tumor stage (327). Focusing on the functional properties of 
lncRNA TUG1, it was proved that lncRNA TUG1 was important in cell cycle regulation of gastric cancer cells, through the interaction of IncRNA TUG1 with either PRC2 complex or cyclin-dependent protein kinase inhibitors such as p15, p16, p21, p27, and p57 (320). Of particular interest was the competitive inhibitory association of TUG1 and miR-381, affecting SRY-Box Transcription Factor 4 (SOX-4) levels and leading to enhanced migratory capacities of gastric cancer cells (328). In another case, it was illustrated that the negative relationship between the lncRNA TUG1 and miR-145-5p accelerated gastric cancer proliferation (347).

In hepatocellular carcinoma, high lncRNA TUG1 expression was tightly related to cancer progression, suggesting the diagnostic importance of the lncRNA TUG1 (311). It was reported that 1 cRNA TUG1 was positively related to the alpha-fetoprotein (AFP) gene. Considering that AFP gene had high prognostic significance in non-hepatitis B/non-hepatitis C HCC (NBNC-HCC), it was plausible to suggest that the lncRNA TUG1 could be effectively used as an unfavorable prognostic marker in patients with non-hepatitis B/non-hepatitis C HCC (NBNC-HCC) (348). At the molecular level, the tumor-promoting role of IncRNA TUG1 was observed to orchestrate hepatocellular carcinoma environment, through the sequestration of distinct miRNAs. Following lncRNA TUG1 silencing, TUG1 inhibited its interactions with various miRNAs, causing their upregulation and the subsequent cancer remission (349). In that direction, it was supported that lncRNA TUG1 was a competitive inhibitor of miR-216b-5p, through the assembly of distal-less homeobox 2 (DLX2), thus leading to exacerbation of hepatocellular carcinoma (HCC) (349), given that overexpression of DLX2 is present in HCC patients with poor prognosis (350). In another study, it was suggested that lncRNA TUG1 was a competitive inhibitor of miR-132-3p and its target (Sox4), as shown in HepG2, Huh7, HccLM3 cells (351). Additional in vitro experiments proved that IncRNA TUG1 could abrogate the expression levels of miR-144, through activation of Janus kinase/signal transducer and activator of transcription 3 (JAK/STAT3), thereby contributing to tumor progression (352). Alternatively, the lncRNA TUG1 could reduce miR-142-3p expression levels, thereby positively contributing to zinc finger e-box binding homeobox 1 (ZEB1)-induced epithelial-mesenchymal transition and metastasis in hepatocarcinoma cells (Huh7 and HepG2) (353). Similarly, it was substantiated that there was an upregulation of TUG1 according to the Barcelona Clinic Liver Cancer (BCLC) stage and tumour size, so that liver tumor carcinogenesis was accelerated through overexpression of lncRNA TUG1 (311). At transcriptional setting, the nuclear transcription factor SP1 induced the mRNA expression of lncRNA TUG1 and in parallel the lncRNA TUG1 functioned as an inhibitor of PRC2 complex, preventing the binding of PRC2 complex to the promoter of Kruppel-like factor 2 (KLF2) (311).

Apart from the sponging action of 1ncRNA TUG1 against miRNAs, it was proved that that lncRNA TUG1 was involved in the regulation of host immune responses during hepatocarcinoma progression. It was highlighted that there was a positive relationship between lncRNA TUG1 and $\mathrm{C}-\mathrm{X}-\mathrm{C}$ chemokine receptor type 4 (CXCR4) protein, accounting for increased infiltration of immune cells such as lymphocytes, neutrophils, and monocytes, through activation of many downstream signaling pathways in the hepatic microenvironment of cancer patients. It was evidenced that elevated CXCR4 expression not only contributed to increased trafficking of immune cells but also was associated with the augmented function of immune cells in tissues isolated from patients with hepatocarcinoma. In particular, the IncRNA TUG1 appeared to increase specific markers in immune populations such as COX-2 in macrophages, C-C chemokine receptor type 7 (CCR7) in neutrophils and CD1c, neuropilin 1 (NRP1), and CD11c in dendritic cells through its positive association with CXCR4 protein (354). Opposite results were observed in catenin $\beta 1$ (CTNNB1)-mutated hepatoblastoma (HB) cells, following lncRNA TUG1 elimination. Behind the molecular mechanism underlying the tight link of lncRNA TUG1 and CXCR4 protein, it was proved that the sponging action of lncRNA TUG1 against miR-335-5p accounted for the acceleration of malignant progression of CTNNB1-mutated HB cells, through increased infiltration of pro-tumor immunocytes (354).

Furthermore, a considerable advance was made in the functional significance of 1ncRNA TUG1 in pancreatic development. Yin et al (302) observed that relatively low levels of TUG1 modulated apoptosis and insulin secretion in pancreatic $\beta$ cells in vitro and in vivo, implying its participation in diabetes pathogenesis. Afterward, researchers investigated the function of TUG1 in malignant transformation of pancreatic cells given that pancreatic cancer (PC) is known as the 'king of cancers' due to the shortage of early diagnostic biomarkers and effective therapeutic methods in advanced stages (355). In pancreatic cancer patients, the expression levels of lncRNA TUG1 appeared to be increased according to the clinical pathologic characteristics. Interestingly, patients with advanced stages (3/4) of pancreatic cancer presented the most important increase of IncRNA TUG1, which correlated with their poor prognosis $(356,357)$. There was a close association of TUG1 with the advanced stage of PC patients and lymphatic metastasis (357), suggesting its significance in the regulatory network that determined the pancreatic development. In another study, overexpression of TUG1 appeared to confer increased gemcitabine chemoresistance in pancreatic ductal adenocarcinoma (PDAC) patients (358), taking into consideration that gemcitabine (2',2'-difluorodeoxycitidine) is the first-line chemotherapy for PDAC patients (359). It was proved that gemcitabine combined with SCH772984 (an inhibitor of the ERK pathway) could counteract the drug resistance driven by the overexpression of lncRNA TUG1, thereby increasing gemcitabine therapeutic efficacy (358).

Additional research findings confirmed that lncRNA TUG1 expression levels culminated in all pancreatic cell lines in vitro, exacerbating tumor progression (356). Some studies proved that sponging action of lncRNA TUG1 against distinct miRNAs accounted for dramatically increased tumor growth of pancreatic cells, facilitating the acquisition of mesenchymal characteristics in tumor cells. For example, lncRNA TUG1 was enriched in three following pancreatic cell lines: SW1990, BxPC3, and PaTu8988 but its levels were discriminated among cell lines, indicating that lncRNA TUG1 exerted its oncogenic role in cell type-dependent manner (343). The 
underlying molecular mechanism of IncRNA TUG1 relied on inhibiting miR-382, thus increasing the recruitment of EZH2 methyltransferase to genes that were implicated in epithelial to mesenchymal transition (EMT) (343).

In another case, the migratory potential of pancreatic cells was validated through the association between lncRNA TUG1 with miR-29c (360). In tissues derived from pancreatic cancer patients, the IncRNA TUG1 appeared to be upregulated, thereby causing the reduced expression levels of tumor suppressor miR-29c and exacerbating pancreatic cancer progression (360). Following the elimination of lncRNA TUG1, the expression levels of miR-29c downstream targets including integrin subunit beta 1 (ITGB1), MMP-2, and MMP-9 were increased, thereby contributing to pancreatic cancer remission (360). In addition, it was shown that the silencing of lncRNA TUG1 prevented pancreatic cancer growth, through inhibition of the Notch1 pathway and upregulation of miR-299-3p. The positive relationship of 1 ncRNA TUG1 with the Notch1 pathway was especially important, given that abnormal activation of the Notch1 pathway accounts for the exacerbation of pancreatic carcinogenesis $(361,362)$. In that regard, the TUG1/miR-299-3p/Notch1 pathway was considered a promising therapeutic approach for pancreatic carcinogenesis. Besides, the IncRNA TUG1 was reported to act as competing endogenous RNA (ceRNAs) to inhibit miR-299-3p, taking into consideration that miR-299-3p has been detected at low levels in thyroid cancer (TC) (363), hepatocellular carcinoma (HCC) (364), and colon cancer (365).

Besides, the oncogenic role of lncRNA TUG1 has been evidenced not only through its potential to promote the acquisition of mesenchymal characteristics by cancer cells, but also through its capacity to interfere with the function of tumor suppressor genes. For example, lncRNA TUG1 was illustrated to be recruited at the promoters of Rho family GTPase 3 (RND3) and metallothionein 2A (MT2A) genes, inhibiting the transcriptional expression of either gene, through the assembly of EZH2 in tissues originated from pancreatic cancer patients (366). The pancreatic cancer growth was worsened through the link between the lncRNA TUG1 and RND3 protein, considering that the RND3 gene is a target of tumor suppressor p53 (367). Conversely, the proliferation of pancreatic cancer cells was prevented by the upregulation of RND3 and the concomitant elimination of lncRNA TUG1 (366). In other words, IncRNA TUG1 has an inverse relationship with the RND3 gene, through the recruitment of EZH2 methyltransferase, which functioned as a scaffold protein, as shown by experiments that were performed in tissues originated from pancreatic cancer patients (366). As a result, the poor prognostic value of lncRNA TUG1 (356) and the tumor-promoting effect of lncRNA TUG1 on cancer invasion (360) raised the possibilities to use lncRNA TUG1 as a novel therapeutic target for combating pancreatic cancer. In that regard, lncRNA TUG1 was presented as a novel potential therapeutic approach, which was extremely helpful in ameliorating pancreatic cancer progression.

The tumor-promoting role of lncRNA TUG1 was proved not only in pancreatic cancer but also it was expanded to other cancer cell types through its interaction with the RND3 gene. In esophageal squamous cell carcinoma (ESCC), the silencing of RND3 was reported to stimulate cell proliferation and cell cycle progression. Conversely, the upregulation of RND3 reversed the phenotype of cancer cells, by preventing cell proliferation and leading to cell cycle arrest at the G0/G1 phase. The aforementioned phenotype of cancer cells was ascribed to the upregulation of RND3 targets including phosphatase and tensin homolog (PTEN) and cyclin-dependent kinase inhibitor $1 \mathrm{~B}\left(\mathrm{CDKN} 1 \mathrm{~B} / \mathrm{p} 27^{\mathrm{kip} 1}\right)$, in combination with downregulation of the signaling molecules such as phosphorylated Akt (pAKT) and cell cycle protein D1 (CCND1)] (134). In glioblastoma, RND3 appeared to be the determinant factor that causes inhibition of CCND1 expression, accompanied by the activation of RB1/retinoblastoma, thereby leading to cancer remission (368).

In colon cancer, it was substantiated that lncRNA TUG1 gathered considerable attention as an oncogene. The overexpression of lncRNA TUG1 was observed in tissues isolated from 88 patients with CRC to a high extent (64.77\%, 57 of 88) compared to that of healthy subjects. To assimilate conditions in a clinical setting, it was revealed that LoVo and SW480 cells presented very high lncRNA TUG1 expression, so a series of functional analysis was focused on those cells (369). Following the silencing of lncRNA TUG1, the growth inhibition of LOVO and SW480 cells was observed and it was attributed to the induction of cell apoptosis (369). In particular, elimination of lncRNA TUG1 significantly induced G0/G1 arrest in CRC cells (LOVO and SW480), disabling them to metastasize (369). When elucidating the importance of the strong tumor-promoting role of lncRNA TUG1, it was evidenced that lncRNA TUG1 caused the constitutive expression of $\mathrm{Wnt} / \beta$-catenin signaling pathway in colon cancer (342). The research group of Jiang (312) also explained that IncRNA TUG1 was the determinant factor of colon cancer proliferation and migration, through its inverse association with tumor suppressor p63. Another research group considered that the upregulation of 1ncRNA TUG1 had strong potential to boost the migration of colon cancer through epithelial-mesenchymal transition (EMT) (369), consistent with the classical tumor-promoting role of lncRNA TUG1 on cancer cell invasion and radioresistance via EMT $(330,332)$. Indeed, TUG1 silencing reduced migration, invasion, the acquisition of mesenchymal characteristics by CRC cells in vitro, and in parallel TUG1 depletion inhibited lung metastasis in vivo (370). In particular, the lncRNA TUG1 targeted its downstream target, the twist-related protein 1 (TWIST1), causing the metastasis of CRC cells to be attenuated via TWIST1 knockdown, independently of TGF- $\beta$ signaling (370). The silencing of lncRNA TUG1 could alleviate all the invasive properties of CRC cells, through inhibition of TGF- $\beta /$ TUG1/TWIST1 signaling cascade (370). Accordingly, Sun et al pointed out that enforced expression of IncRNA TUG1 could be of utmost importance in potentiating invasiveness of colon cancer cells, as TUG1-overexpression SW480 CRC cells formed more metastatic nodules after injection into the spleens of nude mice (371). Following TUG1 upregulation, reduced expression of the epithelial marker E-cadherin and increased expression of mesenchymal markers ( $\mathrm{N}$-cadherin, vimentin, and fibronectin) were detected in colon cancer cells (371). Taken together, the lncRNA TUG1 might serve as a prognostic biomarker and a therapeutic target (371). 
With regard to breast cancer, the role of lncRNA TUG1 is presented as a promising therapeutic target according to tumor staging. Results compiled from TCGA database showed that cancer progression of the patients was markedly improved when lncRNA TUG1 expression levels were increased (341). Tissues originated from 20 triple-negative breast cancer (TNBC), and multiple TNBC cell lines presented low expression levels of lncRNA TUG1, suggesting the tumor-suppressor role of lncRNA TUG1 (341). Interestingly, the therapeutic efficacy of cisplatin was increased through the overexpression of lncRNA TUG1 (341). The underlying mechanism of action of lncRNA TUG1 was based on inhibiting the Wnt signaling pathway through negative regulation of miR-197/Nemo-like kinase (NLK) expression (341). The research group of Wang (372) highlighted that the 1ncRNA TUG1 overexpression was capable of alleviating breast cancer, as demonstrated by experiments in several breast cancer cell lines and in patient samples. In particular, the low lncRNA TUG1 expression was tightly related to mutant p53 expression, as evidenced by results in MDA-MB-231 cancer cells compared to those derived from MCF7 breast cancer cells with wild-type p53 status. In line with that, it was also shown that low lncRNA TUG1 expression was correlated with lymph node metastasis via modulating cell cycle regulators (cyclinD1/CDK4), which were capable of exerting their action as oncogenes in breast cancer (373). On the contrary, Ren et al (347) presented that the 1ncRNA TUG1 functioned as an oncogene, given that TUG1 silencing abrogated breast cancer proliferation, as demonstrated in breast cancer cell lines (MDA-MB-231 and MDA-MB-436). Zhao and Ren (374) delineated the molecular mechanism underlying the tumor-promoting role of lncRNA TUG1, showing that the sponging action of lncRNA TUG1 against miR-9 expression increased the proliferation of $\mathrm{p} 53$ wild type breast cancer cells (such as MCF7 cells). In support of the above, RNA sequencing data derived from TCGA database proved that lncRNA TUG1 was enriched in a great proportion of patients bearing HER2-positive and basal-like subtypes of breast cancer compared to matched controls. Consequently, lncRNA TUG1 might exert a significant prognostic and therapeutic value, monitoring patient responses in pancreatic cancer (375).

Besides, the tumor suppressor role of lncRNA TUG1 was also highlighted in glioma. Most of the studies showed that lncRNA TUG1 was generally downregulated in glioma tissues compared to matched normal tissues (313). When the low 1ncRNA TUG1 expression levels were increased, the glioma progression was counteracted, confirming the tumor suppressor role of lncRNA TUG1. The tumor-blocking action of the lncRNA TUG1 relied on its capacity to sequester miR-26a, leading to enrichment of phosphatase and tensin homolog (PTEN), thus causing inducing mitochondrial apoptosis in glioma cells (313). Even though most of the studies illustrated the downregulation of lncRNA TUG1 in glioma patients, other studies have substantiated the opposite trend. Katsushima et al (338) proved that the lncRNA TUG1 played a significant role in increasing the self-renewal of glioma stem cells by sequestering miR-145 in the cytoplasm and by inhibiting the expression of crucial differentiation genes through the recruitment of PRC2 complex to target genes. The inhibitory action of TUG1 against miR-145, was responsible for the upregulation of SOX 2 and c-Myc expression, thereby promoting self-renewal in glioma stem cells (338). In essence, Notch signaling caused lncRNA TUG1 overexpression in glioma stem cells, thereby increasing the recruitment of PCR2 complex to neuronal differentiation-associated genes and causing their epigenetic silencing (338). In another study, it was shown that IncRNA TUG1 exerted pro-tumorigenic action, not only by triggering glioma progression but also by playing a crucial role in metastasis. The lncRNA TUG1 was considered as an important regulator of tumor angiogenesis, by elevating VEGF expression and potentiating tumor growth via augmenting tumor microvessel density (340). In more depth, the mechanism underlying the angiogenesis-stimulatory action of lncRNA TUG1 was based on inhibiting miR-299 in glioblastoma cells (340).

Since the blood-tumor barrier inhibits the delivery of chemotherapeutic drugs to brain tumor tissue (376), the possibility of TUG1 to increase the movement of chemotherapeutic drugs in brain tissues was especially important. Cai et al (333) highlighted that inhibition of TUG1 enabled chemotherapeutic drugs to be permeabilized through blood-tumor, to deal with glioma progression. In particular, silencing of lncRNA TUG1 increased blood-tumor barrier permeability, through reducing the expression of three junction proteins, namely occludin, tight junction protein-1 (ZO-1) and claudin-5 (333). Elucidating the underlying molecular mechanism of TUG1, it was proved that TUG1 exerted its inhibitory action against miR-144, thereby targeting heat shock transcription factor 2 (HSF2) (333).

Apart from the importance of lncRNA TUG1 in multiple cancer types, its significance was evidenced in both sexdependent cancer types including ovarian and prostate. TCGA database supported that the lncRNA TUG1 was remarkably upregulated in tissues of epithelial ovarian cancer patients compared to paired adjacent tissues. The overexpression of lncRNA TUG1 was positively correlated with pathological grade, tumor size, supporting its high prognostic value $(377,378)$. As a general note, the most known interacting partners of the lncRNA TUG1 were identified as the miRNAs: miR-29c, miR-142, and miR-145 $(332,379,380)$ exacerbating the unrestrained growth of bladder cancer cells. For example, the overexpression of TUG1 was reported to inhibit the miR-29c expression, accounting for the uncontrolled, and the migration of bladder cancer cells (T24 and EJ) (379). It is important to be mentioned that the oncogenic role of lncRNA TUG1 was consistent with that in pancreatic cancer since the IncRNA TUG1 functioned as a tumor promoter in the pancreas through its association with miR-29c (360). Apart from the inhibitory effect of 1ncRNA TUG1 on miR-29c, TUG1 appeared to upregulate zinc finger e-box binding homeobox 2 (ZEB2) transcription factor, through its competitive interaction with miR-142, thereby enabling the increased cell proliferation and migration of bladder cancer cells (380). Likewise, lncRNA TUG1 was shown to function as a potent inhibitor of miR-145 expression, facilitating bladder cancer cell metastasis by increasing the recruitment of ZEB2 transcription factor to target EMT genes, thereby providing new insights into the regulation of radioresistance mediated by lncRNA TUG1 (332). The significance of lncRNA TUG1 was also proved through its capacity to amplify the radiosensitivity of bladder cancer through inhibition of high-mobility group 
protein 1 (HMGB1) expression (381). As a result, the lncRNA TUG1 was proposed as a promising therapeutic target and prognostic marker in bladder cancer.

In that direction, it was shown that overexpression of TUG1 was the most determinant factor for activating the Wnt/ $\beta$-catenin signaling pathway (380) through negative regulation of miR-138-5p and subsequent upregulation of Sirtuin 1 (Sirt1)-nicotinamide adenosine dinucleotide (NAD)dependent deacetylase in CC cells (382). In more depth, the increased activity of SIRT1 protein appeared to be pronounced in inhibiting the expression of epithelial markers such as E-cadherin, in turn exerting a positive effect on the Wnt/ $\beta$-catenin signaling pathway, through a positive feedback loop (382). In line with the above, additional experiments showed that IncRNA TUG1 caused the increased invasion of human umbilical vein endothelial cells (HUVECs), by upregulating leucine-rich alpha-2-glycoprotein 1 (LRG1) secretion through transforming growth factor-beta (TGF- $\beta$ ) pathway (383), confirming its oncogenic role. In particular, both SKOV3 and CAOV3 endothelial cell lines presented strong pro-angiogenic effects via Smad1/5/8 signaling pathways, thereby leading to binding of LRG1 protein to TGF- $\beta$ accessory receptor (384). In this way, tumor-related angiogenesis was reduced after LRG1 elimination, as manifested by the downregulation VEGF-a, angiopoietin-1 (Ang-1), thereby reducing the signal transmission through TGF- $\beta$ pathway (385). Likewise, angiogenesis was demonstrated to be increased in cerebral ischemia, through the positive effect of LRG1 on the TGF- $\beta 1$ pathway (386). If one considered that there was a positive association of LRG1 and VEGF- $\alpha$, it was plausible that lncRNA TUG1 contributed to increased angiogenesis through LRG1 upregulation $(384,385)$. In parallel, lncRNA TUG1 increased hypoxia-inducible factor- $1 \alpha$ (HIF-1 $\alpha$ ) expression, though LRG1 upregulation, by accelerating tumor angiogenesis (387). Besides, it was proved that extracellular taurine triggered angiogenesis, through activation of Akt, extracellular- signal-regulated kinase (ERK), and steroid-receptor-coactivator/focal adhesion kinase (Src/FAK) signaling cascades in vitro and in vivo (388). The cell cycle progression of endothelial cells was regulated by Akt- and ERK-dependent cell signaling pathways and the cell migration of endothelial cells was orchestrated in an Src-dependent manner, without stimulating inflammation and permeability in vitro and in vivo (388).

Hence, the IncRNA TUG1 was identified to predict poor prognosis of prostate cancer patients (389). Many researchers have provided deep insight into the oncogenic role of TUG1, supporting that the function of lncRNA TUG1 was mediated through its capacity to hinder the expression levels of related miRNAs: miR-145-5p, miR-144, and miR-381 (327,328,347). The molecular mechanism of lncRNA TUG1 relied on triggering cancer cell proliferation through its effects on the cell cycle of prostate cancer cells $(320,346)$. Importantly, the lncRNA TUG1 was of critical importance for prostate cancer progression in vitro and in vivo, exerting its action through miR-128-3p/YES1 axis (390). The lncRNA TUG1 elicited its potential oncogenic role in prostate cancer cells, by inhibiting miR-128-3p and its target YES1 (390).

Similarly, abnormal expression of TUG1 was observed to predict poor prognosis of ESCC patients, serving as a potential oncogene (391). In a cohort of 62 patients, the lncRNA TUG1 was significantly overexpressed in ESCC tissues compared with paired adjacent normal tissues, and the high expression level of TUG1 was related to family history and upper segment of esophageal cancer (392). By loss of function experiments, it was substantiated that the silencing of TUG1 limited the proliferation and migration of ESCC cells and arrested the cell cycle progression (392). Behind its molecular targets, it was observed that lncRNA TUG1 potentiated the EMT progression of ESSC cells, though its preventive action on miR-148a-3p (393). The silencing of lncRNA TUG1 was sufficient to reverse all the manifestations of EMT progression, due to its inverse correlation with miR-148a-3p, as shown by experiments in ESCC (EC9706 and OE19) cells (393). The significant regulatory function of TUG1 against the migration of ESCC cells was supported by the fact that expression of EMT-associated proteins (C-myc, Cyclin D1, and catenin-beta $1 / \beta$-catenin) was under the control of antagonistic interaction of TUG1 and miR-148a-3p (393).

In osteosarcoma, the overexpression of lncRNA TUG1 was observed according to tumor size, distant metastasis, TNM staging, and overall and recurrence-free survival of patients, suggesting that lncRNA exhibited strong prognostic value. Interestingly, the lncRNA TUG1 was considered a superior biomarker to alkaline phosphatase (ALP) in distinguishing osteosarcoma patient cases from healthy controls (394). Focusing on its molecular mechanisms, the lncRNA TUG1 functioned as an oncogene in osteosarcoma through its capacity to bind to various miRNAs (395). To begin with, the fact that overexpression of lncRNA TUG1 in osteosarcoma cells was accompanied by the transcriptional inhibition of miR-212-3p, which in turn caused the relative downregulation of forkhead box A1 (FOXA1) which was a transcriptional target of $\mathrm{miR}-212-3 \mathrm{p}$ (396). The negative regulation of miR-212-3p also exerted a great impact on affecting the expression levels of sex-determining region Y box 4 (SOX4) (397), causing the overexpression of SOX4 which comprises an oncogene in various malignancies, including osteosarcoma (398). The latest research findings supported that the IncRNA TUG1 functioned as an endogenous sponge, downregulating either miR-335-5p (399) or miR-9-5p (334) or miR-219a-5p (400) or miR-132-3p (397), thus promoting migration of osteosarcoma cells. Similarly, the lncRNA TUG1 inhibited miR-144-3p, causing nuclear translocation of $\beta$-catenin in osteosarcoma cells (MG63, U2OS, HOS, and Saos-2), thus exacerbating tumor progression (401). In another study, the lncRNA TUG1 functioned as an oncogene, competitively inhibiting miR-219a-5p (400). The attenuating action of lncRNA TUG1 against miR-219a-5p seemed to potentiate osteosarcoma progression, through the activation of either Wnt/ $\beta$-catenin signaling pathway or of Akt signal transduction pathway (400). In addition, it was proved that lncRNA TUG1 acted as an endogenous sponge to directly bind to miR-9-5p, inhibiting its expression. The lncRNA TUG1 overturned the effect of miR-9-5p on the proliferation, colony formation, cell cycle arrest, and apoptosis in osteosarcoma cells, which involved in the activation of POU class 2 homeobox 1 (POU2F1) expression. As a result, a novel TUG1/miR-9-5p/POU2F1 pathway was revealed, in which lncRNA TUG1 acted as a competitive 
endogenous RNA by sponging miR-9-5p, leading to downregulation of POU2F1, thereby worsening the progression of osteosarcoma. These findings corroborate the importance of lncRNA-targeted therapy against human osteosarcoma (137). The importance of lncNRA TUG1 was also confirmed by its involvement in metastasis, given that early osteosarcoma gives rise to robust progression and aggressive metastasis. Indeed, Ma et al (394) supported that TUG1 expression was also correlated to post-operative chemotherapy, tumour size and Enneking surgical stage. As the TUG1 expression levels were increased, lncRNA TUG1 was associated with poorer prognosis, including shortened overall and progression-free survival, independent of other clinicopathological parameters.

At the molecular setting, the lncRNA TUG1 expression was reported to be increased through the TGF- $\beta$ pathway elicited by cancer-associated fibroblasts (CAFs). The impaired TUG1 expression functioned as a miRNA 'sponge' to competitively confer protection to the hypoxia inducible factor -1a (HIF-1a) mRNA 3'UTR from the inhibitory action of miR-143-5p. It was proposed that the IncRNA TUG1 might be a prognostic indicator for osteosarcoma and could be a therapeutic target for osteosarcoma, through its potent significance in determining osteosarcoma cell metastasis, angiogenesis, and proliferation in vivo and in vitro (402). As a result, the high lncRNA TUG1 expression was regarded as a critical modulator in orchestrating the metastasis of osteosarcoma cells.

In acute myeloid leukemia (AML), patients were diagnosed with poor prognosis, when TUG1 expression levels were high. The underlying mechanism of lncRNA TUG1 relied on interfering with miR-34a and recruiting EZH2 methyltransferase, thus rendering HL60/ADR cells insensitive to adriamycin (ADR) (403). As a result, the high expression pattern of lncRNA TUG1 provided a clue about the diagnostic importance of lncRNA TUG1 in ADR resistant cells of AML. Accordingly, it was reported that multiple myeloma patients presented showing significant downregulation of lncRNA TUG1 as opposed to healthy subjects (404).

Cancer progression is affected by aberrant energy metabolism, which can be elicited through the action of lncRNAs (405). It has been reported that cancer progression can be aggravated through abnormal high glycolysis and enhanced glutamine metabolism (406). Taking into consideration that lncRNA TUG1 was an important regulator of mitochondrial bioenergetics (301), further investigations revealed that IncRNA TUG1 was overexpressed in clinical specimens isolated from cholangiocarcinoma (ICC) and its upregulation was dependent on tumor stage (407). Also, its overexpression was considered as an independent prognostic factor for patients with poor outcome (407). Other researchers suggested that lncRNA TUG1 functioned as a competitive endogenous RNA against miR-145, causing metabolic reprogramming of ICC cells (408). In particular, it was proved that the overexpression of TUG1 was capable of elevating glutamine consumption, $\alpha$-Ketoglutaric acid (a-KG) production, and ATP levels through miR-145 inhibition and subsequent Sirt3/GDH elevation (408). As a result, the lncRNA TUG1 might be a useful prognostic biomarker in ICC patients and a potentially important therapeutic target to orchestrate metabolic reprogramming in ICC, thereby recovering the glutamine metabolism in cancer cells. Accordingly, the abnormal overexpression of lncRNA TUG1 appeared to play a crucial role in the progression of osteosarcoma cells, through metabolic alterations (408). The lncRNA TUG1 seemed to affect the osteosarcoma progression through its ability to augment glucose consumption, and lactate production owing to the upregulation of hexokinase-2 (409).

Taken together, many oncologists have embraced the search of molecular mechanisms underlying the therapeutic index of lncRNA TUG1 in a wide spectrum of cancer types, to overcome the challenges such as toxicity, drug resistance, tumor heterogeneity encountered by gold standard treatment decisions. The molecular characterization of TUG1 targets enables physicians to define the genomic changes in each tumor type, thereby contributing to the design of selected tumor-targeted therapy based on the detailed portrait of tumor types. It seems that lncRNA TUG1 signature is heading toward the mainstream in precision medicine given that lncRNAs are characterized by high sensitivity and specificity against their targets (410). Nonetheless, more research is urgently needed to elucidate the crosstalk between lncRNA TUG1 and endogenous molecules, to render the lncRNA TUG1 as an appealing therapeutic approach against cancer.

\section{The association of IncRNA TUG1 and chemoresistance}

During metastasis of cancer cells, the major challenges are related to the biologic heterogeneity of tumor cells and to the tumor microenvironment, which accounts for the drug-resistant phenotype in cancer cells (411-413). Besides, it is important to be noted that the ineffectiveness of multiple chemotherapeutic drugs is attributed to the phenomenon of multidrug resistance (MDR). In this MDR procedure, cancer cells acquire multiple aggressive characteristics, enabling them resistant to the cytostatic or cytotoxic action of potential drugs. Among them, limited drug penetrance; enhanced drug efflux; affected membrane lipids (e.g., ceramides) (414); increased drug metabolism; altered drug targets; detoxification by compartmentalization; blocked programmed cell death (apoptosis); induction of mechanisms that repair DNA damage (415); alterations in the cell cycle and checkpoints have been reported as the most significant barriers during MDR that should be addressed (416). For example, the expression of MDR protein 1 (MDR1) and MDR-associated protein 1 (MRP1) expression can be reduced in DOX or cisplatin-resistant BUC cells, through Wnt/ $\beta$-catenin pathway (417). In this context, the upregulation of the $\mathrm{Wnt} / \beta$-catenin pathway has shown to reverse the effects of lncRNA TUG1 knockdown on Dox resistance in T24/DR cells (418).

To overcome resistance liabilities, research has focused on examining the functional role of IncRNA TUG1. The pleiotropic nature of IncRNA has enabled it to be uniquely tailored, with the ultimate aim of overcoming cancer cell drug resistance originating from a redundancy of oncogenic signaling in a wide spectrum of cancer types. In particular, the lncRNA TUG1 has appeared to play a complicated role in cancer progression, exerting either beneficial or detrimental effects on resistant cancer cells. On one side, the lncRNA TUG1 has been extensively analyzed for its capacity to confer resistance in various types of cancer cells in response to classical 
therapeutic actions. On the other side, the lncRNA TUG1 has been reported as a protective agent that enables cancer cells to overcome resistance conferred by various chemotherapeutic drugs.

In this direction, it has been reported that overexpression of lncRNA TUG1 in esophageal squamous carcinoma cells confers resistance to platinum combined with 5-FU or PTX (391). For example, elevated levels of lncRNA TUG1 appear in bladder urothelial cancer (BUC) patients, indicating the poor response of patients to DOX chemotherapy (418). Activation of the Wnt/ $\beta$-catenin pathway has been shown to contribute to DOX resistance in BUC patients, as demonstrated by TCGA Pan-Cancer (PANCAN) (418). Also, IncRNA TUG1 has been reported to be overexpressed in colon cancer patients, given that lncRNA TUG1 silencing is regarded as an effective way to overcome MTX resistance conferred by colorectal cancer cells. TUG1/miR-186/CPEB2 is the main signaling cascade that determines the reduced sensitivity of CRC cells to MTX (419).

It has also been proven that lncRNA TUG1 significantly contributes to ADR resistance in urothelial carcinoma of the bladder (UCB) growth, thanks to its positive relationship with master transcription factor of anti-oxidant response NF-erythroid 2 (NF-E2)-related factor 2 (Nrf2) (403). Deficiency of either transcription factor Nrf2 or lncRNA TUG1 enables UCB cells to overcome ADR-based chemotherapy resistance (403). The overexpression of TUG1 also plays an important role in inducing PTX resistance in both SK-OV-3 and A2780/R ovarian cancer cells, exerting its effect on increasing autophagy. In particular, lncRNA TUG1 promotes autophagy, through its inhibitory effect on miR-29b-3p, resulting in conferring PTX resistance to both SK-OV-3 and A2780/R ovarian cancer cells (420). The PTX resistance of ovarian cancer cells can be overcome through the silencing of TUG1 (420). The potential of lncRNA TUG1 to increase autophagy in ovarian cancer cells is considered one of the important mechanisms by which the lncRNA TUG1 inactivates PTX therapeutic effect, thereby enabling ovarian cancer cells resistant to PTX (421).

In addition to the above, $\mathrm{CC}$ tissues have presented increased expression levels of TUG1 and the expression levels of lncRNA TUG1 are tightly associated with cisplatin (cis-Dichlorodiammineplatinum, DDP). The silencing of IncRNA TUG1 seems to hinder the proliferative rate of CC cells, through the increased signal transmission of the MAPK pathway (p38 MAPK, JNK), thereby accelerating the apoptosis of CC cells (422). Following MAPK activation, the expression levels of the RFX7 gene (transcriptional target of TUG1) are inhibited, due to TUG1 silencing in CC cells. The contribution of IncRNA TUG1 is of utmost importance because the majority of patients commonly develop cisplatin resistance (423). Besides, it is important to highlight that overexpression of TUG1 has a high prognostic value in CC patients (423).

Similarly, ADR-resistant AML tissues and cells have presented increased sensitivity to ADR response, through TUG1 silencing. It has been documented that there is a competitive indirect interaction between lncRNA TUG1 and miR-34a, through the action of one component of Polycomb complex (EZH2), which catalyzes the epigenetic regulation of
miR-34a. The increased sensitivity of AML cells to ADR is accompanied by the silencing of IncRNA TUG1 as well as the upregulation of miR-34a (403).

Consistent with the above, lncRNA TUG1 has been reported to confer resistance to small cell lung cancer (SCLC) cells through modulation of the expression of LIMK2b (a splice variant of LIM-kinase 2) via binding with EZH2 (424). Similarly, Xu et al observed that lncRNA TUG1 elimination facilitated the cisplatin sensitivity of cisplatin-resistant ESCC (ECA109 or EC9706) cells (425). The upregulation of TUG1 appeared to exacerbate cancer progression given that TUG1 IncRNA is an epigenetic inhibitor of PDCD4 expression through recruitment of EZH2 (426). In the same context, the up-regulation of TUG1 was also identified to drive increased migration of pancreatic ductal adenocarcinoma cells, thereby reducing the gemcitabine chemosensitivity (358).

Last but not least, Liu et al (427) examined the expression levels of various lncRNAs including TUG1 in glioma cell lines U87 and U251 upon treatment with resveratrol and DOX (427). It was demonstrated that the expression pattern of lncRNA TUG1 was downregulated upon necrosis induction in both cell lines but it was remained unchanged during DNA damage-induced apoptosis.

\section{The role of taurine or IncRNA TUG1 as a prognostic marker}

Towards evaluating new therapeutic agents, multiple studies are underway identifying specific markers, that might provide indicative clues for the increased susceptibility of cancer patients to specific drugs and the design of a suitable personalized medicine. Some criteria such as reliability, reproducibility, noninvasiveness, simplicity, and cost-efficiency are required to identify the ideal biomarker. In samples of physiologic fluids, taurine can be easily isolated to be used for diagnostic scopes. Taurine or lncRNA TUG1 can provide us with specific information concerning an individual's nutrition or disease status or medications when taurine or lncRNA TUG1 is harvested from physiological fluids. As high-technology platforms become available, the major question remains whether taurine or lncRNA TUG1 can be used as a good prognostic biomarker or diagnostic indicator to guide care for cancer patients.

Accumulating evidence has supported that TUG1 lncRNA has a prognostic significance in cancer patients. The lncRNA TUG1 protein expression levels have been associated with clinicopathologic features of patients harboring various tumor types, indicating that TUG1 lncRNA is a strong indicator of poor outcome in cancer patients. Interestingly, results of meta-analysis derived from nine cancer types, have proposed that there is an inverse relationship between lncRNA TUG1 and overall survival in patients with cancer. In this regard, TUG1 is linked to the unfavorable overall survival of cancer patients given that TUG1 expression is increased depending on the severity of clinicopathological symptoms of cancer patients and can be used as a new reliable biomarker for cancer patients (428). These data suggest that lncRNA TUG1 can be added as a potential biomarker to the growing list of tests for the management of cancer patients. However, some limitations should not be addressed in the meta-analysis due to the involvement of 12 studies. To acquire more reliable data, more 
large-scale and well-designed studies need to be included in the analysis to circumvent the existed heterogeneity across 12 studies, as demonstrated by inconsistent threshold values of TUG1 lncRNA protein expression (428).

With regards to taurine, proton MRS technique has shown that there is a differential expression in 29 metabolites between mice bearing B16F10 melanoma cells and control matched mice. Among the metabolites with differential expression between tumor and normal samples, taurine is involved. As a result, taurine can be leveraged for either monitoring tumor progression or evaluating the pharmacological efficacy of different therapeutic schemes (234). As a result, the introduction of taurine or lncRNA TUG1 heralds its use as a predictive biomarker, by which correct patient stratification can be accomplished during cancer therapy. The correct tumor patient sampling is mandatory for predictive biomarker discovery.

\section{Conclusions}

In the past decade, there has been a tremendous advance in our knowledge on the molecular mechanisms of taurine or its haloamines against inflammatory disorders and distinct cancer types. To manipulate taurine or its haloamines for therapeutic purposes, it is essential to elucidate how the complex cellular interplay between stimulatory and inhibitory signals is regulated. In this direction, the anti-inflammatory or anti-cancer effect elicited by taurine or its haloamines has been evaluated, thus delineating the exact molecular mechanism caused by either agent. In addition, some studies have presented that taurine functions as a favorable agent for cancer chemoprevention, used either alone or in combination with other drugs, by maximizing the therapeutic outcome of chemotherapeutic drugs without increasing cytotoxicity. At present, the most alluring reason to recommend taurine supplementation is a taurine deficiency. Advances in the understanding of cell procedures regulated by taurine or its haloamines are needed to provide significant input to ignite our minds, ensuring a bright future regarding the potential therapeutic use of taurine and its derivatives in cancer and inflammation.

LncRNA TUG1 is regarded as a potential therapeutic target or prognostic marker, exerting its biological action at least in part through chromatin remodeling and sequestration of microRNAs. The lncRNA TUG1 is presented to be an eminent non-coding RNA of utmost importance to modulate targets, either enriching or reliably attenuating gene expression. Despite the great accrual of research findings, there is a certain paucity pertinent to the detailed downstream molecular mechanisms mediated by TUG1. Certain research technologies, such as high-throughput identification of binding partners and integrative analysis of omics data can be employed to elucidate the functional role of lncRNA TUG1 in a cell type-dependent manner. Further analysis will provide us with information that will enable the prognostic significance of TUG1 and the systemic modulation of TUG1 as a therapeutic option against distinct cancer types. To use the lncRNA TUG1 as a therapeutic target, some barriers should be bypassed such as the increased vulnerability of IncRNA to degradation and its low efficiency of delivery.

Taurine or its haloamines or its lncRNA TUG1 deserve further research studies that will substantiate into their functional properties, their specificity, their stability, their toxicity, prompting researchers to optimize either agent for implementation in the clinical setting.

\section{Acknowledgements}

Not applicable.

\section{Funding}

This study was supported by I.K.Y State Scholarship Foundation for S. Baliou's Ph.D. studies. The IKY code is 2018-050-0502-13155.

\section{Availability of data and materials}

Not applicable.

\section{Authors' contributions}

All the authors were involved in the conception and design of the study. SB performed the literature search, wrote the manuscript, critically analyzing the existing knowledge and designed the figures; SB, AMK, DAS and VZ also contributed to editing the manuscript. All authors approved the final manuscript.

\section{Ethics approval and consent to participate}

Not applicable.

\section{Patient consent for publication}

Not applicable.

\section{Competing interests}

DAS is the Editor-in-Chief for the journal, but had no personal involvement in the reviewing process, or any influence in terms of adjudicating on the final decision, for this article. The other authors declare that they have no competing interests.

\section{References}

1. Leon R, Wu H, Jin Y, Wei J, Buddhala C, Prentice H and Wu JY: Protective function of taurine in glutamate-induced apoptosis in cultured neurons. J Neurosci Res 87: 1185-1194, 2009.

2. Chang CY, Shen CY, Kang CK, Sher YP, Sheu WHH, Chang CC and Lee TH: Taurine protects HK-2 cells from oxidized LDL-induced cytotoxicity via the ROS-mediated mitochondrial and p53-related apoptotic pathways. Toxicol Appl Pharmacol 279: 351-363, 2014.

3. Schaffer S, Azuma J, Takahashi K and Mozaffari M: Why is taurine cytoprotective? Adv Exp Med Biol 526: 307-321, 2003.

4. Marcinkiewicz $J$ and Kontny E: Taurine and inflammatory diseases. Amino Acids 46: 7-20, 2014.

5. Schuller-Levis GB and Park E: Taurine and its chloramine: Modulators of immunity. Neurochem Res 29: 117-126, 2004.

6. Maher SG, Condron CE, Bouchier-Hayes DJ and Toomey DM: Taurine attenuates CD3/interleukin-2-induced T cell apoptosis in an in vitro model of activation-induced cell death (AICD). Clin Exp Immunol 139: 279-286, 2005.

7. Fukuda K, Hirai Y, Yoshida H, Nakajima T and Usui T: Free amino acid content of lymphocytes and granulocytes compared. Clin Chem 28: 1758-1761, 1982. 
8. Capuozzo E, Pecci L, Baseggio Conrado A and Fontana M: Thiotaurine prevents apoptosis of human neutrophils: A putative role in inflammation. Adv Exp Med Biol 775: 227-236, 2013.

9. Redmond HP, Stapleton PP, Neary P and Bouchier-Hayes D: Immunonutrition: The role of taurine. Nutrition 14: 599-604, 1998.

10. William R, Watson G, Redmond HP, Wang JH and Bouchier-Hayes D: Mechanisms involved in sodium arsenite-induced apoptosis of human neutrophils. J Leukoc Biol 60: 625-632, 1996

11. Condron CM, Toomey DM, Casey RG, Creagh $\mathrm{T}$ and Bouchier-Hayes DJ: Taurine protects against PMN dysfunction and death in urine. Urol Res 32: 338-345, 2004.

12. Masuda M, Horisaka K and Koeda T: Effects of taurine on neutrophil function in hyperlipidemic rats. Jpn J Pharmacol 40: 478-480, 1986

13. Schaffer SW, Azuma J and Mozaffari M: Role of antioxidant activity of taurine in diabetes. Can J Physiol Pharmacol 87: 91-99, 2009.

14. Wang L, Zhao N, Zhang F, Yue W and Liang M: Effect of taurine on leucocyte function. Eur J Pharmacol 616: 275-280, 2009.

15. Pasantes-Morales $\mathrm{H}$ and Cruz $\mathrm{C}$ : Taurine and hypotaurine inhibit light-induced lipid peroxidation and protect rod outer segment structure. Brain Res 330: 154-157, 1985

16. Son M, Kim HK, Kim WB, Yang J and Kim BK: Protective effect of taurine on indomethacin-induced gastric mucosal injury. Adv Exp Med Biol 403: 147-155, 1996

17. Marcinkiewicz J, Grabowska A, Bereta J and Stelmaszynska T: Taurine chloramine, a product of activated neutrophils, inhibits in vitro the generation of nitric oxide and other macrophage inflammatory mediators. J Leukoc Biol 58: 667-674, 1995.

18. Nakajima Y, Osuka K, Seki Y, Gupta RC, Hara M, Takayasu M and Wakabayashi $\mathrm{T}$ : Taurine reduces inflammatory responses after spinal cord injury. J Neurotrauma 27: 403-410, 2010.

19. Zhang F, Mao Y, Qiao H, Jiang H, Zhao H, Chen X, Tong L and Sun X: Protective effects of taurine against endotoxin-induced acute liver injury after hepatic ischemia reperfusion. Amino Acids 38: 237-245, 2010.

20. Elson CO, Sartor RB, Tennyson GS and Riddell RH: Experimental models of inflammatory bowel disease. Gastroenterology 109: 1344-1367, 1995.

21. Abdih H, Kelly CJ, Bouchier-Hayes D, Barry M and Kearns S Taurine prevents interleukin-2-induced acute lung injury in rats. Eur Surg Res 32: 347-352, 2000.

22. Sun M, Zhao Y, Gu Y and Xu C: Anti-inflammatory mechanism of taurine against ischemic stroke is related to down-regulation of PARP and NF- $\kappa B$. Amino Acids 42: 1735-1747, 2012.

23. Son MW, Ko JI, Doh HM, Kim WB, Park TS, Shim MJ and Kim BK: Protective effect of taurine on TNBS-induced inflammatory bowel disease in rats. Arch Pharm Res 21: 531-536, 1998

24. Shimizu M, Zhao Z, Ishimoto Y and Satsu H: Dietary taurine attenuates dextran sulfate sodium (DSS)-induced experimental colitis in mice. Adv Exp Med Biol 643: 265-271, 2009.

25. Zhao Z, Satsu H, Fujisawa M, Hori M, Ishimoto Y, Totsuka M, Nambu A, Kakuta S, Ozaki H and Shimizu M: Attenuation by dietary taurine of dextran sulfate sodium-induced colitis in mice and of THP-1-induced damage to intestinal Caco-2 cell monolayers. Amino Acids 35: 217-224, 2008

26. Yin Y, Wen K, Wu Y, Kang Y and Lou J: Inhibition of sodium current by taurine magnesium coordination compound prevents cesium chloride-induced arrhythmias. Biol Trace Elem Res 146 192-198, 2012

27. Wang Q, Fan W, Cai Y, Wu Q, Mo L, Huang Z and Huang H: Protective effects of taurine in traumatic brain injury via mitochondria and cerebral blood flow. Amino Acids 48: 2169-2177, 2016

28. Megaraj V, Iida T, Jungsuwadee P, Hofmann AF and Vore M: Hepatobiliary disposition of 3alpha,6alpha,7alpha,12alpha-tetrahydroxy-cholanoyl taurine: A substrate for multiple canalicular transporters. Drug Metab Dispos 38: 1723-1730, 2010.

29. Guler L, Tavlasoglu M, Yucel O, Guler A, Sahin MA, Kurkluoglu M, Sirin Y, Eken A, Gamsizkan M, Dakak M, et al: Taurine attenuates lung ischemia-reperfusion injury after lung transplantation in rats. J Anesth 28: 347-353, 2014

30. Park SH,Lee H,Park KK,KimHW and Park T: Taurine-responsive genes related to signal transduction as identified by cDNA microarray analyses of HepG2 cells. J Med Food 9: 33-41, 2006.

31. Song XD, Chen CZ, Dong B, Shi YY, Zhang W, Yan LS and Luo GA: Study on the intervening mechanism of taurine on streptozotocin-induced diabetic cataracts. Zhonghua Yan Ke Za Zhi 39: 605-609, 2003 (In Chinese)
32. Schuller-Levis G, Mehta PD, Rudelli R and Sturman J: Immunologic consequences of taurine deficiency in cats. J Leukoc Biol 47: 321-331, 1990.

33. Sapronov NS, Khnychenko LK and Polevshchikov AV: Effects of new taurine derivatives on primary immune response in rats. Bull Exp Biol Med 131: 142-144, 2001

34. Negoro $\mathrm{S}$ and Hara $\mathrm{H}$ : The effect of taurine on the age-related decline of the immune response in mice: The restorative effect on the T cell proliferative response to costimulation with ionomycin and phorbol myristate acetate. Adv Exp Med Biol 315: 229-239; 1992

35. Tappaz ML: Taurine biosynthetic enzymes and taurine transporter: Molecular identification and regulations. Neurochem Res 29: 83-96, 2004

36. Park E, Park SY, Dobkin C and Schuller-Levis G: A novel cysteine sulfinic acid decarboxylase knock-out mouse: Comparison between newborn and weanling mice. Adv Exp Med Biol 803: $3-16,2015$.

37. Vogelstein B and Kinzler KW: Cancer genes and the pathways they control. Nat Med 10: 789-799, 2004.

38. Luo J, Solimini NL and Elledge SJ: Principles of cancer therapy: Oncogene and non-oncogene addiction. Cell 136: 823-837, 2009.

39. Sardesai VM: Role of antioxidants in health maintenance. Nutr Clin Pract 10: 19-25, 1995.

40. Szatrowski TP and Nathan CF: Production of large amounts of hydrogen peroxide by human tumor cells. Cancer Res 51 794-798, 1991 .

41. Trachootham D, Alexandre J and Huang P: Targeting cancer cells by ROS-mediated mechanisms: A radical therapeutic approach? Nat Rev Drug Discov 8: 579-591, 2009.

42. Zhang X, Tu S, Wang Y, Xu B and Wan F: Mechanism of taurine-induced apoptosis in human colon cancer cells. Acta Biochim Biophys Sin (Shanghai) 46: 261-272, 2014.

43. Liu Z, Xia Y, Zhang X, Liu L, Tu S, Zhu W, Yu L, Wan H, Yu B and Wan F: Roles of the MST1-JNK signaling pathway in apoptosis of colorectal cancer cells induced by Taurine. Libyan $\mathrm{J}$ Med 13: 1500346, 2018

44. Tu S, Zhang XL, Wan HF, Xia YQ, Liu ZQ, Yang XH and Wan FS: Effect of taurine on cell proliferation and apoptosis human lung cancer A549 cells. Oncol Lett 15: 5473-5480, 2018.

45. Wang AS, Lodi A, Rivera LB, Izquierdo-Garcia JL, Firpo MA, Mulvihill SJ, Tempero MA, Bergers G and Ronen SM: HR-MAS MRS of the pancreas reveals reduced lipid and elevated lactate and taurine associated with early pancreatic cancer. NMR Biomed 27: 1361-1370, 2014.

46. Opstad KS, Bell BA, Griffiths JR and Howe FA: Taurine: A potential marker of apoptosis in gliomas. Br J Cancer 100: 789-794, 2009

47. Yu J and Kim AK: Effect of taurine on antioxidant enzyme system in B16F10 melanoma cells. Adv Exp Med Biol 643: 491-499, 2009

48. Vanitha MK, Anandakumar P and Sakthisekaran D: Taurine abrogates mammary carcinogenesis through induction of apoptosis in Sprague-Dawley rats. J Biochem Mol Toxicol 32: e22204, 2018.

49. Vanitha MK, Baskaran K, Periyasamy K, Selvaraj S, Ilakkia A, Saravanan D, Venkateswari R, Revathi Mani B, Anandakumar P and Sakthisekaran D: Modulatory effect of taurine on 7,12-Dimethylbenz(a)Anthracene-induced alterations in detoxification enzyme system, membrane bound enzymes, glycoprotein profile and proliferative cell nuclear antigen in rat breast tissue. J Biochem Mol Toxicol 30: 414-423, 2016.

50. Zhang X, Lu H, Wang Y, Liu C, Zhu W, Zheng S and Wan F: Taurine induces the apoptosis of breast cancer cells by regulating apoptosis-related proteins of mitochondria. Int J Mol Med 35: 218-226, 2015.

51. Choi EJ, Tang Y, Lee CB, Cheong SH, Sung SH, Oh MR, Young Jang SY, Park PJ and Kim EK: Effect of taurine on in vitro migration of MCF-7 and MDA-MB-231 human breast carcinoma cells. Adv Exp Med Biol 803: 191-201, 2015.

52. He F, Ma N, Midorikawa K, Hiraku Y, Oikawa S, Zhang Z, Huang G, Takeuchi K and Murata M: Taurine exhibits an apoptosis-inducing effect on human nasopharyngeal carcinoma cells through PTEN/Akt pathways in vitro. Amino Acids 50: 1749-1758, 2018

53. Tang Y, Choi E-J, Cheong SH, Hwang YJ, Arokiyaraj S, Park PJ, Moon SH and Kim EK: Effect of taurine on prostate-specific antigen level and migration in human prostate cancer cells. Adv Exp Med Biol 803: 203-214, 2015.

54. Chatzakos V, Slätis K, Djureinovic T, Helleday T and Hunt MC: $\mathrm{N}$-acyl taurines are anti-proliferative in prostate cancer cells. Lipids 47: 355-361, 2012. 
55. Li H, Ruan WJ, Liu LQ, Wan HF, Yang XH, Zhu WF, Yu LH, Zhang XL and Wan FS: Impact of taurine on the proliferation and apoptosis of human cervical carcinoma cells and its mechanism. Chin Med J (Engl) 132: 948-956, 2019.

56. Srivastava S, Roy R, Singh S, Kumar P, Dalela D and Sankhwar SN: Taurine - a possible fingerprint biomarker in non-muscle invasive bladder cancer: A pilot study by $1 \mathrm{H}$ NMR spectroscopy. Cancer Biomark 6: 11-20, 2010.

57. Zhang X, Du W, Shen F and Wang J: Research on effects of taurine on the transplanted tumor of mice. Wei Sheng Yan Jiu 26: 321-324, 1997 (In Chinese)

58. Yousef HN and Aboelwafa HR: The potential protective role of taurine against 5-fluorouracil-induced nephrotoxicity in adult male rats. Exp Toxicol Pathol 69: 265-274, 2017.

59. Vanitha MK, Priya KD, Baskaran K, Periyasamy K, Saravanan D, Venkateswari R, Mani BR, Ilakkia A, Selvaraj S, Menaka R, et al Taurine regulates mitochondrial function during 7,12-dimethyl Benz[a]anthracene induced experimental mammary carcinogenesis. J Pharmacopuncture 18: 68-74, 2015.

60. Tu S, Zhang X, Luo D, Liu Z, Yang X, Wan H, Yu L, Li H and Wan F: Effect of taurine on the proliferation and apoptosis of human hepatocellular carcinoma HepG2 cells. Exp Ther Med 10: 193-200, 2015.

61. Sadzuka Y, Matsuura M and Sonobe T: The effect of taurine, a novel biochemical modulator, on the antitumor activity of doxorubicin. Biol Pharm Bull 32: 1584-1587, 2009.

62. Daigeler A, Chromik AM, Geisler A, Bulut D, Hilgert C, Krieg A, Klein-Hitpass L, Lehnhardt M, Uhl W and Mittelkötter U: Synergistic apoptotic effects of taurolidine and TRAIL on squamous carcinoma cells of the esophagus. Int J Oncol 32: 1205-1220, 2008.

63. Jacobi CA, Menenakos C and Braumann C: Taurolidine - a new drug with anti-tumor and anti-angiogenic effects. Anticancer Drugs 16: 917-921, 2005.

64. Rodak R, Kubota H, Ishihara H, Eugster HP, Könü D, Möhler H, Yonekawa Y and Frei K: Induction of reactive oxygen intermediates-dependent programmed cell death in human malignant ex vivo glioma cells and inhibition of the vascular endothelial growth factor production by taurolidine. J Neurosurg 102 1055-1068, 2005.

65. Refai NS, Louka ML, Halim HY and Montasser I: Long non-coding RNAs (CASC2 and TUG1) in hepatocellular carcinoma: Clinical significance. J Gene Med 21: e3112, 2019.

66. Matés JM, Segura JA, Alonso FJ and Márquez J: Oxidative stress in apoptosis and cancer: An update. Arch Toxicol 86: 1649-1656, 2012

67. Mates JM, Segura JA, Alonso FJ and Marquez J: Sulphurcontaining non enzymatic antioxidants: Therapeutic tools against cancer. Front Biosci (Schol Ed) 4: 722-748, 2012

68. Okamoto K, Sugie S, Ohnishi M, Makita H, Kawamori T, Watanabe T, Tanaka T and Mori H: Chemopreventive effects of taurine on diethylnitrosamine and phenobarbital-induced hepatocarcinogenesis in male F344 rats. Jpn J Cancer Res 87: 30-36, 1996

69. Abd-Allah AR, Gado AM, Al-Majed AA, Al-Yahya AA and Al-Shabanah OA: Protective effect of taurine against cyclophosphamide-induced urinary bladder toxicity in rats. Clin Exp Pharmacol Physiol 32: 167-172, 2005.

70. Islambulchilar M, Asvadi I, Sanaat Z, Esfahani A and Sattari M: Effect of taurine on attenuating chemotherapy-induced adverse effects in acute lymphoblastic leukemia. J Cancer Res Ther 11: 426-432. 2015

71. Desai TK, Maliakkal J, Kinzie JL, Ehrinpreis MN, Luk GD and Cejka J: Taurine deficiency after intensive chemotherapy and/or radiation. Am J Clin Nutr 55: 708-711, 1992.

72. Tabassum H, Parvez S, Rehman H, Dev Banerjee B, Siemen D and Raisuddin S: Nephrotoxicity and its prevention by taurine in tamoxifen induced oxidative stress in mice. Hum Exp Toxicol 26: 509-518, 2007

73. Tabassum H, Rehman H, Banerjee BD, Raisuddin S and Parvez S: Attenuation of tamoxifen-induced hepatotoxicity by taurine in mice. Clin Chim Acta 370: 129-136, 2018.

74. Parvez S, Tabassum H, Banerjee BD and Raisuddin S: Taurine prevents tamoxifen-induced mitochondrial oxidative damage in mice. Basic Clin Pharmacol Toxicol 102: 382-387, 2008.

75. Han X, Yue J and Chesney RW: Functional TauT protects against acute kidney injury. J Am Soc Nephrol 20: 1323-1332, 2009.

76. Al-Asmari A, Al-Zahrani A, Khan A, Al-Shahrani H and Ali Al Amri M: Taurine ameliorates 5-flourouracil-induced intestinal mucositis, hepatorenal and reproductive organ damage in Wistar rats: A biochemical and histological study. Hum Exp Toxicol 35: 10-20, 2018
77. Das J, Ghosh J, Manna P and Sil PC: Taurine protects rat testes against doxorubicin-induced oxidative stress as well as p53, Fas and caspase 12-mediated apoptosis. Amino Acids 42: 1839-1855, 2012.

78. Das J, Ghosh J, Manna P and Sil PC: Taurine suppresses doxorubicin-triggered oxidative stress and cardiac apoptosis in rat via up-regulation of PI3-K/Akt and inhibition of p53, p38-JNK Biochem Pharmacol 81: 891-909, 2011.

79. Neary PM, Hallihan P, Wang JH, Pfirrmann RW, BouchierHayes DJ and Redmond HP: The evolving role of taurolidine in cancer therapy. Ann Surg Oncol 17: 1135-1143, 2010.

80. Mobley JA and Brueggemeier RW: Estrogen receptor-mediated regulation of oxidative stress and DNA damage in breast cancer. Carcinogenesis 25: 3-9, 2004

81. Huang S, Chong N, Lewis NE, Jia W, Xie G and Garmire LX: Novel personalized pathway-based metabolomics models reveal key metabolic pathways for breast cancer diagnosis. Genome Med 8: 34, 2016.

82. Sitter B, Bathen TF, Singstad TE, Fjøsne HE, Lundgren S, Halgunset $\mathbf{J}$ and Gribbestad IS: Quantification of metabolites in breast cancer patients with different clinical prognosis using HR MAS MR spectroscopy. NMR Biomed 23: 424-431, 2010.

83. El Agouza IM, Eissa SS, El Houseini MM, El-Nashar DE and Abd El Hameed OM: Taurine: A novel tumor marker for enhanced detection of breast cancer among female patients. Angiogenesis 14: 321-330, 2011.

84. He YU, Li QQ and Guo SC: Taurine attenuates dimethylbenz[a] anthracene-induced breast tumorigenesis in rats: A plasma metabolomic study. Anticancer Res 36: 533-543, 2016.

85. Zhou DN, Deng YF, Li RH, Yin P and Ye CS: Concurrent alterations of RAGE, RECK, and MMP9 protein expression are relevant to Epstein-Barr virus infection, metastasis, and survival in nasopharyngeal carcinoma. Int J Clin Exp Pathol 7: 3245-3254, 2014

86. Shennan D and Thomson J: Estrogen regulation and ion dependence of taurine uptake by MCF-7 human breast cancer cells. Cell Mol Biol Lett 12: 396-406, 2007.

87. Pine MJ, Kim U and Ip C: Free amino acid pools of rodent mammary tumors. J Natl Cancer Inst 69: 729-735, 1982.

88. Brown NS and Bicknell R: Hypoxia and oxidative stress in breast cancer. Oxidative stress: Its effects on the growth, metastatic potential and response to therapy of breast cancer. Breast Cancer 3: 323-327, 2001.

89. Ambrosone CB, Marshall JR, Vena JE, Laughlin R, Graham S, Nemoto T and Freudenheim JL: Interaction of family history of breast cancer and dietary antioxidants with breast cancer risk (New York, United States). Cancer Causes 6: 407-415, 1995.

90. Freudenheim JL, Marshall JR, Vena JE, Laughlin R, Brasure JR, Swanson MK, Nemoto T and Graham S: Premenopausal breast cancer risk and intake of vegetables, fruits, and related nutrients. J Natl Cancer Inst 88: 340-348, 1996.

91. Kubota A, Meguid MM and Hitch DC: Amino acid profiles correlate diagnostically with organ site in three kinds of malignant tumors. Cancer 69: 2343-2348, 1992.

92. Maeda J,Higashiyama M,Imaizumi A,Nakayama T, YamamotoH, Daimon T, Yamakado M, Imamura F and Kodama K: Possibility of multivariate function composed of plasma amino acid profiles as a novel screening index for non-small cell lung cancer: A case control study. BMC Cancer 10: 690, 2010.

93. Satsu H, Ishimoto Y, Nakano T, Mochizuki T, Iwanaga $T$ and Shimizu M: Induction by activated macrophage-like THP-1 cells of apoptotic and necrotic cell death in intestinal epithelial Caco-2 monolayers via tumor necrosis factor-alpha. Exp Cell 312: 3909-3919, 2006

94. Wang H, Tso VK, Slupsky CM and Fedorak RN: Metabolomics and detection of colorectal cancer in humans: A systematic review. Future Oncol 6: 1395-1406, 2010.

95. Scheel C, Eaton EN, Li SH, Chaffer CL, Reinhardt F, Kah KJ, Bell G, Guo W, Rubin J, Richardson AL and Weinberg RA: Paracrine and autocrine signals induce and maintain mesenchymal and stem cell states in the breast. Cell 145: 926-940, 2011.

96. Turman MV, Kingsley PJ, Rouzer CA, Cravatt BF and Marnett LJ: Oxidative metabolism of a fatty acid amide hydrolase-regulated lipid, arachidonoyltaurine. Biochemistry 47: 3917-3925, 2008.

97. McKinney MK and Cravatt BF: Structure and function of fatty acid amide hydrolase. Annu Rev 74: 411-432, 2005.

98. Saghatelian A, McKinney MK, Bandell M, Patapoutian A and Cravatt BF: A FAAH-regulated class of $\mathrm{N}$-acyl taurines that activates TRP ion channels. Biochemistry 45: 9007-9015, 2006. 
99. Ueki I, Roman HB, Hirschberger LL, Junior C and Stipanuk MH: Extrahepatic tissues compensate for loss of hepatic taurine synthesis in mice with liver-specific knockout of cysteine dioxygenase. Am J Physiol Endocrinol Metab 302: E1292-E1299, 2012.

100. Timbrell JA, Seabra V and Waterfield CJ: The in vivo and in vitro protective properties of taurine. Gen Pharmacol 26: 453-462, 1995.

101. Heidari R, Babaei H and Eghbal MA: Ameliorative effects of taurine against methimazole-induced cytotoxicity in isolated rat hepatocytes. Sci Pharm 80: 987-999, 2012.

102. Boşgelmez İI, Söylemezoğlu T and Güvendik G: The protective and antidotal effects of taurine on hexavalent chromium-induced oxidative stress in mice liver tissue. Biol Trace Elem Res 125: 46-58, 2008

103. Heidari R, Babaei H and Eghbal MA: Amodiaquine-induced toxicity in isolated rat hepatocytes and the cytoprotective effects of taurine and/or N-acetyl cysteine. Res Pharm Sci 9: 97-105, 2014.

104. Sinha M, Manna P and Sil PC: Taurine, a conditionally essential amino acid, ameliorates arsenic-induced cytotoxicity in murine hepatocytes. Toxicol In Vitro 21: 1419-1428, 2007.

105. Vissers MC and Fantone JC: Inhibition of hypochlorous acid-mediated reactions by desferrioxamine. Implications for the mechanism of cellular injury by neutrophils. Free Radic Biol Med 8: 331-337, 1990.

106. Riordan JD, Feddersen CR, Tschida BR, Beckmann PJ Keng VW, Linden MA, Amin K, Stipp CS, Largaespada DA and Dupuy AJ: Chronic liver injury alters driver mutation profiles in hepatocellular carcinoma in mice. Hepatology 67: 924-939, 2018.

107. Glauert HP, Calfee-Mason K, Stemm DN, Tharappel JC and Spear BT: Dietary antioxidants in the prevention of hepatocarcinogenesis: A review. Mol Nutr Food Res 54: 875-896, 2010.

108. Bansal AK, Trivedi R, Soni GL and Bhatnagar D: Hepatic and renal oxidative stress in acute toxicity of $\mathrm{N}$-nitrosodiethylamine in rats. Indian J Exp Biol 38: 916-920, 2000.

109. Kang JS, Wanibuchi H, Morimura K, Gonzalez FJ and Fukushima S: Role of CYP2E1 in diethylnitrosamine-induced hepatocarcinogenesis in vivo. Cancer Res 67: 11141-11116, 2007.

110. Başaran-Küçükgergin C, Bingül I, Tekkeşin MS, Olgaç V, Doğru-Abbasoğlu S and Uysal M: Effects of carnosine, taurine, and betaine pretreatments on diethylnitrosamine-induced oxidative stress and tissue injury in rat liver. Toxicol Ind Health 32: 1405-1413, 2016.

111. Chang YY, Chou CH, Chiu CH, Yang KT, Lin YL, Weng WL and Chen YC: Preventive effects of taurine on development of hepatic steatosis induced by a high-fat/cholesterol dietary habit. J Agric Food Chem 59: 450-457, 2011.

112. Kalaz EB, Çoban J, Aydın AF, Doğan-Ekici I, DoğruAbbasoğlu S, Öztezcan S and Uysal M: Carnosine and taurine treatments decreased oxidative stress and tissue damage induced by D-galactose in rat liver. J Physiol Biochem 70: 15-25, 2014.

113. Kerai MD, Waterfield CJ, Kenyon SH, Asker DS and Timbrell JA The effect of taurine depletion by beta-alanine treatment on the susceptibility to ethanol-induced hepatic dysfunction in rats. Alcohol 36: 29-38, 2001

114. You JS and Chang KJ: Taurine protects the liver against lipid peroxidation and membrane disintegration during rat hepatocarcinogenesis. Adv Exp Med Biol 442: 105-112, 1998.

115. Liu Y, Li F, Zhang L, Wu J, Wang Y and Yu H: Taurine alleviates lipopolysaccharide-induced liver injury by anti-inflammation and antioxidants in rats. Mol Med Rep 16: 6512-6517, 2017.

116. Seabra V, Stachlewitz RF and Thurman RG: Taurine blunts LPS-induced increases in intracellular calcium and TNF-alpha production by Kupffer cells. J Leukoc Biol 64: 615-621, 1998.

117. Wu G, Yang Q, Yu Y, Lin S, Feng Y, Lv Q, Yang J and Hu J: Taurine inhibits kupffer cells activation induced by lipopolysaccharide in alcoholic liver damaged rats. Adv Exp Med Biol 975 789-800, 2017.

118. Kim SK and Kim YC: Attenuation of bacterial lipopolysaccharide-induced hepatotoxicity by betaine or taurine in rats. Food Chem Toxicol 40: 545-549,2002.

119. Abd-Rabou AA, Zoheir KMA and Ahmed HH: Potential impact of curcumin and taurine on human hepatoma cells using Huh-7 cell line. Clin 45: 1519-1521, 2012.

120. El-Houseini ME, El-Agoza IA, Sakr MM and El-Malky GM: Novel protective role of curcumin and taurine combination against experimental hepatocarcinogenesis. Exp Ther Med 13: $29-36,2017$
121. Kim YS, Cheong SH, Hwang JW, Lodhi G, Lee KH, Choi DK, Song H, Lee SH, Park DJ, Ahn CB, et al: Effect of taurine on viability and proliferation of murine melanoma B16F10 cells. Adv Exp Med Bio 803: 167-177, 2015.

122. Finnegan N, Toomey D, Condron C, Redmond HP, Da Costa M and Bouchier-Hayes DJ: Potentiation of the therapeutic index of interleukin-2 immunotherapy by combination with taurine in a syngeneic murine tumour model. Ir J Med Sci 171: 85-88, 2002.

123. Son YI, Dallal RM and Lotze MT: Combined treatment with interleukin-18 and low-dose interleukin-2 induced regression of a murine sarcoma and memory response. J Immunother Hagerstown Md 26: 234-240, 2003.

124. Da Costa ML, Redmond HP and Bouchier-Hayes DJ: Taurolidine improves survival by abrogating the accelerated development and proliferation of solid tumors and development of organ metastases from circulating tumor cells released following surgery. J Surg Res 101: 111-119, 2001.

125. Logotheti S, Khoury N, Vlahopoulos SA, Skourti E, Papaevangeliou D, Liloglou T, Gorgoulis V, Budunova I, Kyriakopoulos AM and Zoumpourlis V: N-bromotaurine surrogates for loss of antiproliferative response and enhances cisplatin efficacy in cancer cells with impaired glucocorticoid receptor. Transl Res 173: 58-73.e2, 2016.

126. Gottardi W and Nagl M: N-chlorotaurine, a natural antiseptic with outstanding tolerability. J Antimicrob Chemother 65: 399-409, 2010.

127. Carr C, Ng J and Wigmore T: The side effects of chemotherapeutic agents. Curr Anaesth Crit Care 19: 70-79, 2008.

128. Bergkvist K and Wengström Y: Symptom experiences during chemotherapy treatment-With focus on nausea and vomiting. Eur J Oncol Nurs 10: 21-29, 2006.

129. Kim KS, Tsuji M, Kimura T and Sezaki H: Effect of taurine on the gastrointestinal absorption of drugs - Ionic requirement for the action. J Pharmacobiodyn 5: 172-178, 1982.

130. Zeybek A, Ercan F, Çetinel Ş, Çikler E, Sağlam B and Sener G: Taurine ameliorates water avoidance stress-induced degenerations of gastrointestinal tract and liver. Dig Dis Sci 51: 1853-1861, 2006.

131. Abe M, Takahashi M, Takeuchi K and Fukuda M: Studies on the significance of taurine in radiation injury. Radiat Res 33: 563, 1968.

132. Sener G, Sehirli O, Cetinel S, Midillioğlu S, Gedik N and Ayanoğlu-Dülger G: Protective effect of taurine against alendronate-induced gastric damage in rats. Fundam Clin Pharmacol 19: 93-100, 2005 .

133. Saransaari P and Oja SS: Taurine and neural cell damage. Amino Acids 19: 509-526, 2000.

134. Waters E, Wang JH, Redmond HP, Wu QD, Kay E and Bouchier-Hayes D: Role of taurine in preventing acetaminophen-induced hepatic injury in the rat. Am J Physiol Gastrointest Liver Physiol 280: G1274-G1279, 2001.

135. Erdem A, Gündogan NÜ, Usubütün A, Kılınç K, Erdem ŞR, Kara A and Bozkurt A: The protective effect of taurine against gentamicin-induced acute tubular necrosis in rats. Nephrol Dial Transplant 15: 1175-1182, 2000

136. Piao J, Meng F, Fang H, Piao F, Jin B, Li M and Li W: Effect of taurine on thymus differentiation of dex-induced immunosuppressive mice. Adv Exp Med Biol 1155: 381-390, 2019.

137. Hamaguchi T, Azuma J, Awata N, Ohta H, Takihara K, Harada H, Kishimoto S and Sperelakis N: Reduction of doxorubicin-induced cardiotoxicity in mice by taurine. Res Commun Chem Pathol Pharmacol 59: 21-30, 1988.

138. Refik Mas M, Comert B, Oncu K, Vural SA, Akay C, Tasci I, Ozkomur E, Serdar M, Mas N, Alcigir G and Yener N: The effect of taurine treatment on oxidative stress in experimental liver fibrosis. Hepatol Res 28: 207-215, 2004.

139. Kato T, Tsunekawa M, Wang S, Yamashita T and Ma N: Effect of taurine on iNOS-mediated DNA damage in drug-induced renal injury. Adv Exp Med Biol 975: 717-727, 2017.

140. Gewirtz D: A critical evaluation of the mechanisms of action proposed for the antitumor effects of the anthracycline antibiotics adriamycin and daunorubicin. Biochem Pharmacol 57: 727-741, 1999.

141. Milic V and Dragojevic V: Doxorubicin-induced oxidative injury of cardiomyocytes - do we have right strategies for prevention? In: Cardiotoxicity of oncologic treatments. Fiuza M, (ed). InTech, 2012. http://www.intechopen. $\mathrm{com} /$ books/cardiotoxicity-of-oncologic-treatments/doxorubicininduced-oxidative-injury-of-cardiomyocytes-do-we-have-rightstrategies-for-prevention-. 
142. Ujhazy P, Zaleskis G, Mihich E, Ehrke MJ and Berleth ES Doxorubicin induces specific immune functions and cytokine expression in peritoneal cells. Cancer Immunol Immunother 52: 463-472, 2003.

143. Kim YS, Kim EK, Hwang JW, Kim WS, Shin WB, Natarajan SB, Moon SH, Jeon BT and Park PJ: Taurine attenuates doxorubicin-induced toxicity on B16F10 cells. Adv Exp Med Biol 975: 1179-1190, 2017.

144. Grenier MA and Lipshultz SE: Epidemiology of anthracycline cardiotoxicity in children and adults. Semin Oncol 25 (Suppl 10): S72-S85, 1998.

145. Volkova M and Russell R III: Anthracycline cardiotoxicity: Prevalence, pathogenesis and treatment. Curr Cardiol Rev 7: 214-220, 2012.

146. Comereski CR, Peden WM, Davidson TJ, Warner GL, Hirth RS and Frantz JD: BR96-doxorubicin conjugate (BMS-182248) versus doxorubicin: A comparative toxicity assessment in rats. Toxicol Pathol 22: 473-488, 1994

147. Nagai K, Fukuno S, Oda A and Konishi H: Protective effects of taurine on doxorubicin-induced acute hepatotoxicity through suppression of oxidative stress and apoptotic responses. Anticancer Drugs 27: 17-23, 2016.

148. Kim YS, Sung SH, Tang Y, Choi EJ, Choi YJ, Hwang YJ, Park PJ and Kim EK: Protective effect of taurine on mice with doxorubicin-induced acute kidney injury. Adv Exp Med Biol 975: 1191-1201, 2017

149. Adedara IA, Ojuade TJD, Olabiyi BF, Idris UF, Onibiyo EM, Ajeigbe OF and Farombi EO: Taurine ameliorates renal oxidative damage and thyroid dysfunction in rats chronically exposed to fluoride. Biol Trace Elem Res 175: 388-395, 2017.

150. Mohamed RH, Karam RA and Amer MG: Epicatechin attenuates doxorubicin-induced brain toxicity: Critical role of TNF- $\alpha$, iNOS and NF-кB. Brain Res Bull 86: 22-28, 2011.

151. Gradishar WJ and Vokes EE: 5-Fluorouracil cardiotoxicity: A critical review. Ann Oncol 1: 409-414, 1990.

152. D'Souza UJ and Narayana K: Induction of seminiferous tubular atrophy by single dose of 5-fluorouracil (5-FU) in Wistar rats. Indian J Physiol Pharmacol 45: 87-94, 2001.

153. Narayana K, D'Souza UJ, Sanyal AK and Rao KP: 5-fluorouracil (5-FU) induces the formation of giant cells and sloughing of seminiferous epithelium in the rat testis. Indian J Physiol Pharmacol 44: 317-322, 2000.

154. Cheah KY, Howarth GS, Yazbeck R, Wright TH, Whitford EJ, Payne C, Butler RN and Bastian SE: Grape seed extract protects IEC-6 cells from chemotherapy-induced cytotoxicity and improves parameters of small intestinal mucositis in rats with experimentally-induced mucositis. Cancer Biol Ther 8: 382-390, 2009.

155. Son JY, Shin JW, Wang JH, Park HJ, Kim HG, Raghavendran HR and Son CG: Chemotherapy-induced myelotoxicity and incidence of lung metastasis in an animal model. Hum Exp Toxicol 30: 649-655, 2011.

156. Tsibiribi P, Bui-Xuan C, Bui-Xuan B, Lombard-Bohas C, Duperret S, Belkhiria M, Tabib A, Maujean G, Descotes J and Timour Q: Cardiac lesions induced by 5-fluorouracil in the rabbit. Hum Exp Toxicol 25: 305-309, 2006.

157. Rashid S, Ali N, Nafees S, Hasan SK and Sultana S: Mitigation of 5-Fluorouracil induced renal toxicity by chrysin via targeting oxidative stress and apoptosis in wistar rats. Food Chem Toxicol 66: 185-193, 2014.

158. Heidari R, Rasti M, Shirazi Yeganeh B, Niknahad H, Saeedi A and Najibi A: Sulfasalazine-induced renal and hepatic injury in rats and the protective role of taurine. Bioimpacts 6: 3-8, 2016.

159. Han X: Targeting Taurine Transporter (TauT) for cancer immunotherapy of p 53 mutation mediated cancers - molecular basis and preclinical implication. Adv Exp Med Biol 1155: 543-553, 2019.

160. Nazarewicz RR, Zenebe WJ, Parihar A, Larson SK, Alidema E, Choi J and Ghafourifar P: Tamoxifen induces oxidative stress and mitochondrial apoptosis via stimulating mitochondrial nitric oxide synthase. Cancer Res 67: 1282-1290, 2007.

161. Deng X, Liang J, Lin ZX, Wu FS, Zhang YP and Zhang ZW: Natural taurine promotes apoptosis of human hepatic stellate cells in proteomics analysis. World $\mathrm{J}$ Gastroenterol 16: 1916-1923, 2010.

162. Alam SS, Hafiz NA and Abd El-Rahim AH: Protective role of taurine against genotoxic damage in mice treated with methotrexate and tamoxfine. Environ Toxicol Pharmacol 31: 143-152, 2011

163. Fontanelli R, Spatti G, Raspagliesi F, Zunino F and Di Re F: A preoperative single course of high-dose cisplatin and bleomycin with glutathione protection in bulky stage IB/II carcinoma of the cervix. Ann Oncol 3: 117-122, 1992.
164.Panici PB, Greggi S, Scambia G, Ragusa G, Baiocchi G, Battaglia F, Coronetta F and Mancuso S: High-dose cisplatin and bleomycin neoadjuvant chemotherapy plus radical surgery in locally advanced cervical carcinoma: A preliminary report. Gynecol Oncol 41: 212-216, 1991.

165. Pabla N and Dong Z: Cisplatin nephrotoxicity: Mechanisms and renoprotective strategies. Kidney Int 73: 994-1007, 2008.

166. Ries F and Klastersky J: Nephrotoxicity induced by cancer chemotherapy with special emphasis on cisplatin toxicity. Am J Kidney Dis 8: 368-379, 1986.

167. Townsend DM, Deng M, Zhang L, Lapus MG and Hanigan MH: Metabolism of cisplatin to a nephrotoxin in proximal tubule cells. J Am Soc Nephrol 14: 1-10, 2003.

168. Francescato HDC, Costa RS, Scavone C and Coimbra TM: Parthenolide reduces cisplatin-induced renal damage. Toxicology 230: 64-75, 2007.

169. Yao X, Panichpisal K, Kurtzman N and Nugent K: Cisplatin nephrotoxicity: A review. Am J Med Sci 334: 115-124, 2007.

170. Chtourou Y, Aouey B, Aroui S, Kebieche M and Fetoui H: Anti-apoptotic and anti-inflammatory effects of naringin on cisplatin-induced renal injury in the rat. Chem Biol Interact 243: $1-9,2016$.

171. Torre LA, Trabert B, DeSantis CE, Miller KD, Samimi G, Runowicz CD, Gaudet MM, Jemal A and Siegel RL: Ovarian cancer statistics, 2018: Ovarian cancer statistics, 2018. CA Cancer J Clin 68: 284-296, 2018.

172. Han X and Chesney RW: Regulation of TauT by cisplatin in LLC-PK1 renal cells. Pediatr Nephrol 20: 1067-1072, 2005.

173. Shalby AB, Assaf N and Ahmed HH: Possible mechanisms for $\mathrm{N}$-acetyl cysteine and taurine in ameliorating acute renal failure induced by cisplatin in rats. Toxicol Mech Methods 21: 538-546, 2011.

174. Kim T and Kim AK: Taurine enhances anticancer activity of cisplatin in human cervical cancer cells. Adv Exp Med Biol 776: 189-198, 2013

175. Tsunekawa M, Wang S, Kato $\mathrm{T}$, Yamashita $\mathrm{T}$ and $\mathrm{Ma} \mathrm{N}$ : Taurine administration mitigates cisplatin induced acute nephrotoxicity by decreasing DNA damage and inflammation: An Immunocytochemical Study. Adv Exp Med Biol 975: 703-716, 2017.

176. Sørensen BH, Thorsteinsdottir UA and Lambert IH: Acquired cisplatin resistance in human ovarian A2780 cancer cells correlates with shift in taurine homeostasis and ability to volume regulate. Am J Physiol Cell Physiol 307: C1071-C1080, 2014.

177. Sugiura H, Okita S, Kato T, Naka T, Kawanishi S, Ohnishi S, Oshida $\mathrm{Y}$ and Ma N: Protection by taurine against INOS-dependent DNA damage in heavily exercised skeletal muscle by inhibition of the NF- $\kappa B$ signaling pathway. Adv Exp Med Biol 775: 237-246, 2013.

178. Ma N, Kato T, Isogai T, Gu Y and Yamashita T: The potential effects of taurine in mitigation of radiation nephropathy. Adv Exp Med Biol 1155: 497-505, 2019.

179. Owoeye O, Adedara IA and Farombi EO: Pretreatment with taurine prevented brain injury and exploratory behaviour associated with administration of anticancer drug cisplatin in rats. Biomed Pharmacother 102: 375-384, 2018.

180. Bishnu A, Sakpal A, Ghosh N, Choudhury P, Chaudhury K and Ray P: Long term treatment of metformin impedes development of chemoresistance by regulating cancer stem cell differentiation through taurine generation in ovarian cancer cells. Int J Biochem Cell Biol 107: 116-127, 2019.

181. Badary OA: Taurine attenuates fanconi syndrome induced by ifosfamide without compromising its antitumor activity. Oncol Res 10: 355-360, 1998.

182. Han X and Chesney RW: The role of taurine in renal disorders. Amino Acids 43: 2249-2263, 2012.

183. Bouckenooghe T, Remacle C and Reusens B: Is taurine a functional nutrient? Curr Opin Clin Nutr Metab Care 9: 728-733, 2006.

184. Dotan E, Aggarwal C and Smith MR: Impact of rituximab (Rituxan) on the treatment of B-cell Non-Hodgkin's lymphoma. P T 35: 148-157, 2010.

185. Han YM, Awng N, Nu LH, Thway NM and McLiesh P: Orthopaedic nursing in developing nations: A collaboration between the Republic of the Union of Myanmar (Burma) and Australia. Int J Orthop Trauma Nurs 27: 41-45, 2017.

186. Dong JF, Zheng X-Q and Rui HB: Effect of taurine on immune function in mice with T-cell lymphoma during chemotherapy. Asian Pac J Trop Med 10: 1090-1094, 2017.

187. D'souza M, Jaimini A, Bansal A, Tripathi M, Sharma R, Mondal A and Tripathi RP: FDG-PET/CT in lymphoma. Indian J Radiol Imaging 23: 354-365, 2013. 
188. Marcinkiewicz J, Grabowska A, Bereta J, Bryniarski K and Nowak B: Taurine chloramine down-regulates the generation of murine neutrophil inflammatory mediators. Immunopharmacology 40: 27-38, 1998.

189. Klebanoff SJ: Myeloperoxidase-halide-hydrogen peroxide antibacterial system. J Bacteriol 95: 2131-2138, 1968

190. Thomas EL: Myeloperoxidase-hydrogen peroxide-chloride antimicrobial system: Effect of exogenous amines on antibacterial action against Escherichia coli. Infect Immun 25: 110-116, 1979.

191. Babior BM: Oxygen-dependent microbial killing by phagocytes (first of two parts). N Engl J Med 298: 659-668, 1978.

192. Henderson JP, Byun J, Williams MV, Mueller DM, McCormick ML and Heinecke JW: Production of brominating intermediates by myeloperoxidase. A transhalogenation pathway for generating mutagenic nucleobases during inflammation. J Biol Chem 276: 7867-7875, 2001.

193. Klebanoff SJ: Myeloperoxidase: Friend and foe. J Leukoc Biol 77: 598-625, 2005.

194. van Dalen CJ and Kettle AJ: Substrates and products of eosinophil peroxidase. Biochem J 358: 233-239, 2001.

195. Weiss SJ, Klein R, Slivka A and Wei M: Chlorination of taurine by human neutrophils. Evidence for hypochlorous acid generation. J Clin Invest 70: 598-607, 1982

196. Marcinkiewicz J, Strus M, Walczewska M, Machul A and Mikołajczyk D: Influence of taurine haloamines ( $\mathrm{TauCl}$ and $\mathrm{TauBr}$ ) on the development of pseudomonas aeruginosa biofilm: A preliminary study. Adv Exp Med Biol 775: 269-283, 2013.

197. Tokunaga S, Kanayama A and Miyamoto Y: Modification of IkappaBalpha by taurine bromamine inhibits tumor necrosis factor alpha-induced NF-kappaB activation. Inflamm Res 56 479-486, 2007.

198. Midwinter RG, Peskin AV, Vissers MC and Winterbourn CC: Extracellular oxidation by taurine chloramine activates ERK via the epidermal growth factor receptor. J Biol Chem 279: 32205-32211, 2004

199. Grisham MB, Jefferson MM, Melton DF and Thomas EL: Chlorination of endogenous amines by isolated neutrophils. Ammonia-dependent bactericidal, cytotoxic, and cytolytic activities of the chloramines. J Biol Chem 259: 10404-10413, 1984.

200. Thomas EL, Grisham MB and Jefferson MM: Myeloperoxidasedependent effect of amines on functions of isolated neutrophils. J Clin Invest 72: 441-454, 1983.

201. Kim C and Kang IS: Taurine chloramine, a taurine metabolite from activated neutrophils, inhibits osteoclastogenesis by suppressing NFATc1 expression. Adv Exp Med Biol 803 99-107, 2015.

202. Walczewska M, Peruń A, Białecka A, Sróttek M, Jamróz W, Dorożyński P, Jachowicz R, Kulinowski P, Nagl M, Gottardi W and Marcinkiewicz J: Comparative analysis of microbicidal and anti-inflammatory properties of novel taurine bromamine derivatives and bromamine T. Adv Exp Med Biol 975: 515-534, 2017.

203. Gottardi W, Hagleitner M and Nagl M: N,N-Dichlorotaurine: Chemical and bactericidal properties. Arch Pharm (Weinheim) 338: 473-483, 2005.

204. Gottardi W and Nagl M: Chlorine covers on living bacteria: The initial step in antimicrobial action of active chlorine compounds. J Antimicrob Chemother 55: 475-482, 2005.

205. Roos D, Eckmann CM and Yazdanbakhsh M: Killing of schistosomula by taurine chloramine and taurine bromamine. Am J Trop Med Hyg 37: 106-110, 1987.

206. Nagl M,Nguyen VA, Gottardi W, UlmerH and Höpfl R: Tolerability and efficacy of N-chlorotaurine in comparison with chloramine $\mathrm{T}$ for the treatment of chronic leg ulcers with a purulent coating: A randomized phase II study. Br J Dermatol 149: 590-597, 2003.

207. Marcinkiewicz J, Wojas-Pelc A, Walczewska M, LipkoGodlewska S, Jachowicz R, Maciejewska A, Białecka A and Kasprowicz A: Topical taurine bromamine, a new candidate in the treatment of moderate inflammatory acne vulgaris: A pilot study. Eur J Dermatol 18: 433-439, 2008.

208. Nagl M, Hess MW, Pfaller K, Hengster P and Gottardi W: Bactericidal activity of micromolar N-chlorotaurine: Evidence for its antimicrobial function in the human defense system. Antimicrob Agents Chemother 44: 2507-2513, 2000.

209. Nagl M, Teuchner B, Pöttinger E, Ulmer H and Gottardi W: Tolerance of $\mathrm{N}$-chlorotaurine, a new antimicrobial agent, in infectious conjunctivitis - a phase II pilot study. Ophthalmologica 214 $111-114,2000$
210. Neher A, Gstöttner M, Nagl M, Scholtz A and Gunkel AR: N-chlorotaurine - a new safe substance for postoperative ear care. Auris Nasus Larynx 34: 19-22, 2007.

211. Neher A, Nagl M, Appenroth E, Gstöttner M, Wischatta M, Reisigl F, Schindler M, Ulmer H and Stephan K: Acute otitis externa: Efficacy and tolerability of n-chlorotaurine, a novel endogenous antiseptic agent. Laryngoscope 114: 850-854, 2004

212. Mainnemare A, Mégarbane B, Soueidan A, Daniel A and Chapple IL: Hypochlorous acid and taurine-N-monochloramine in periodontal diseases. J Dent Res 83: 823-831, 2004.

213. Marcinkiewicz J: Taurine bromamine (TauBr) - its role in immunity and new perspectives for clinical use. J Biomed Sci 17 (Suppl 1): S3, 2010.

214. Eitzinger C, Ehrlenbach S, Lindner H, Kremser L, Gottardi W, Debabov D, Anderson M, Nagl M and Orth D: N-chlorotaurine, a long-lived oxidant produced by human leukocytes, inactivates Shiga toxin of enterohemorrhagic Escherichia coli. PLoS One 7: e47105, 2012.

215. Kim C, Jang JS, Cho MR, Agarawal SR and Cha YN: Taurine chloramine induces heme oxygenase-1 expression via Nrf2 activation in murine macrophages. Int Immunopharmacol 10: 440-446, 2010.

216. Schuller-Levis GB and Park E: Taurine: New implications for an old amino acid. FEMS Microbiol Lett 226: 195-202, 2003.

217. Green TR, Fellman JH, Eicher AL and Pratt KL: Antioxidant role and subcellular location of hypotaurine and taurine in human neutrophils. Biochim Biophys Acta 1073: 91-97, 1991.

218. Jeon SH, Lee MY, Rahman MM, Kim SJ, Kim GB, Park SY, Hong CU, Kim SZ, Kim JS and Kang HS: The antioxidant, taurine reduced lipopolysaccharide (LPS)-induced generation of ROS, and activation of MAPKs and Bax in cultured pneumocytes. Pulm Pharmacol Ther 22: 562-566, 2009.

219. Oliveira MWS, Minotto JB, de Oliveira MR, Zanotto-Filho A Behr GA, Rocha RF, Moreira JC and Klamt F: Scavenging and antioxidant potential of physiological taurine concentrations against different reactive oxygen/nitrogen species. Pharmacol Rep 62: 185-193, 2010.

220. Marcinkiewicz J, Mak M, Bobek M, Biedroń R, Białecka A, Koprowski M, Kontny E and Maśliński W: Is there a role of taurine bromamine in inflammation? Interactive effects with nitrite and hydrogen peroxide. Inflamm Res 54: 42-49, 2005.

221. Kontny E, Chorąży-Massalska M, Rudnicka W, Marcinkiewicz J and Maśliński W: Comparison of taurine chloramine and taurine bromamine effects on rheumatoid arthritis synoviocytes. Amino Acids 32: 447-452, 2007

222. Park E, Schuller-Levis G, Jia JH and Quinn MR: Preactivation exposure of RAW 264.7 cells to taurine chloramine attenuates subsequent production of nitric oxide and expression of iNOS mRNA. J Leukoc Biol 61: 161-166, 1997.

223. Park E, Jia J, Quinn MR and Schuller-Levis G: Taurine chloramine inhibits lymphocyte proliferation and decreases cytokine production in activated human leukocytes. Clin Immunol 102: 179-184, 2002

224. Kontny E, Maśliński W and Marcinkiewicz J: Anti-inflammatory activities of taurine chloramine: Implication for immunoregulation and pathogenesis of rheumatoid arthritis. Adv Exp Med Biol 526: 329-340, 2003.

225. Olszanecki R and Marcinkiewicz J: Taurine chloramine and taurine bromamine induce heme oxygenase-1 in resting and LPS-stimulated J774.2 macrophages. Amino Acids 27: 29-35, 2004.

226. Kim K, Choi HM, Oh D, Kim C, Jeong JS, Yoo M and Yang HI: Effect of taurine chloramine on the production of matrix metalloproteinases (MMPs) in adiponectin- or IL-1beta-stimulated fibroblast-like synoviocytes. J Biomed Sci 17 (Suppl 1): S27, 2010.

227. Olszanecki R, Kurnyta M, Biedroń R, Chorobik P, Bereta M and Marcinkiewicz J: The role of heme oxygenase-1 in down regulation of PGE2 production by taurine chloramine and taurine bromamine in J774.2 macrophages. Amino Acids 35: 359-364, 2008.

228. Araujo JA, Zhang M and Yin F: Heme oxygenase-1, oxidation, inflammation, and atherosclerosis. Front Pharmacol 3: 119, 2012 .

229. Idelman G, Smith DLH and Zucker SD: Bilirubin inhibits the up-regulation of inducible nitric oxide synthase by scavenging reactive oxygen species generated by the toll-like receptor 4 -dependent activation of NADPH oxidase. Redox Biol 5 : 398-408, 2015 .

230. Jong CJ, Azuma J and Schaffer S: Mechanism underlying the antioxidant activity of taurine: Prevention of mitochondrial oxidant production. Amino Acids 42: 2223-2232, 2012 
231. Tallan HH, Jacobson E, Wright CE, Schneidman K and Gaull GE: Taurine uptake by cultured human lymphoblastoid cells. Life Sci 33: 1853-1860, 1983.

232. Kim C, Chung JK, Jeong JM, Chang YS, Lee YJ, Kim YJ, Lee MC, Koh CS and Kim BK: Uptake of taurine and taurine chloramine in murine macrophages and their distribution in mice with experimental inflammation. Adv Exp Med Biol 442: 169-176, 1998

233. Kwaśny-Krochin B, Bobek M, Kontny E, Gluszko P, Biedroń R, Chain BM, Maśliński W and Marcinkiewicz J: Effect of taurine chloramine, the product of activated neutrophils, on the development of collagen-induced arthritis in DBA $1 / \mathrm{J}$ mice. Amino Acids 23: 419-426, 2002.

234. Chung Y-L, Wassif WS, Bell JD, Hurley M and Scott DL: Urinary levels of creatine and other metabolites in the assessment of polymyositis and dermatomyositis. Rheumatology (Oxford) 42: 298-303, 2003.

235. Kim H, Jeon H, Kong H, Yang Y, Choi B, Kim YM, Neckers L and Jung Y: A molecular mechanism for the anti-inflammatory effect of taurine-conjugated 5-aminosalicylic acid in inflamed colon. Mol Pharmacol 69: 1405-1412, 2006.

236. Quinn MR, Park E and Schuller-Levis G: Taurine chloramine inhibits prostaglandin E2 production in activated raw 264.7 cells by post-transcriptional effects on inducible cyclooxygenase expression. Immunol Lett 50: 185-188, 1996.

237. Park E, Schuller-Levis G and Quinn MR: Taurine chloramine inhibits production of nitric oxide and TNF-alpha in activated RAW 264.7 cells by mechanisms that involve transcriptional and translational events. J Immunol 154: 4778-4784, 1995.

238. Kim C, Park E, Quinn MR and Schuller-Levis G: The production of superoxide anion and nitric oxide by cultured murine leukocytes and the accumulation of TNF-alpha in the conditioned media is inhibited by taurine chloramine. Immunopharmacology 34: 89-95, 1996.

239. Barua M, Liu Y and Quinn MR: Taurine chloramine inhibits inducible nitric oxide synthase and TNF-alpha gene expression in activated alveolar macrophages: Decreased NF-kappaB activation and IkappaB kinase activity. J Immunol 167: 2275-2281, 2001.

240.Park E, Quinn MR, Wright CE and Schuller-Levis G: Taurine chloramine inhibits the synthesis of nitric oxide and the release of tumor necrosis factor in activated RAW 264.7 cells. J Leukoc Biol 54: 119-124, 1993.

241. Sun Jang J, Piao S, Cha YN and Kim C: Taurine chloramine activates Nrf2, increases HO-1 expression and protects cells from death caused by hydrogen peroxide. J Clin Biochem Nutr 45: 37-43, 2009.

242. Fazzino F, Obregón F, Morles M, Rojas A, Arocha L, Mata S and Lima L: Taurine transporter in lymphocytes of patients with major depression treated with venlafaxine plus psychotherapy. Adv Exp Med Biol 643: 217-224, 2009.

243. Wirleitner B, Neurauter G, Nagl M and Fuchs D: Down-regulatory effect of $\mathrm{N}$-chlorotaurine on tryptophan degradation and neopterin production in human PBMC. Immunol Lett 93: 143-149, 2004.

244.Kanayama A, Inoue JI, Sugita-Konishi Y, Shimizu M and Miyamoto Y: Oxidation of Ikappa Balpha at methionine 45 is one cause of taurine chloramine-induced inhibition of NF-kappa B activation. J Biol Chem 277: 24049-24056, 2002.

245. Liu Y and Quinn MR: Chemokine production by rat alveolar macrophages is inhibited by taurine chloramine. Immunol Lett 80: 27-32, 2002.

246. Cobb MH, Xu S, Hepler JE, Hutchison M, Frost J and Robbins DJ: Regulation of the MAP kinase cascade. Cell Mol Biol Res 40: 253-256, 1994.

247. Crews CM, Alessandrini A and Erikson RL: The primary structure of MEK, a protein kinase that phosphorylates the ERK gene product. Science 258: 478-480, 1992.

248. Kim JW and Kim C: Inhibition of LPS-induced NO production by taurine chloramine in macrophages is mediated though Ras-ERK-NF-kappaB. Biochem Pharmacol 70: 1352-1360, 2005.

249. Bhat NR, Zhang P, Lee JC and Hogan EL: Extracellular signal-regulated kinase and p38 subgroups of mitogen-activated protein kinases regulate inducible nitric oxide synthase and tumor necrosis factor-alpha gene expression in endotoxin-stimulated primary glial cultures. J Neurosci 18: 1633-1641, 1998.

250. Ajizian SJ, English BK and Meals EA: Specific inhibitors of p38 and extracellular signal-regulated kinase mitogen-activated protein kinase pathways block inducible nitric oxide synthase and tumor necrosis factor accumulation in murine macrophages stimulated with lipopolysaccharide and interferon-gamma. J Infect Dis 179: 939-944, 1999.
251. Chen CC and Wang JK: p38 but not p44/42 mitogen-activated protein kinase is required for nitric oxide synthase induction mediated by lipopolysaccharide in RAW 264.7 macrophages. Mol Pharmacol 55: 481-488, 1999.

252. Kontny E, Szczepańska K, Kowalczewski J, Kurowska M, Janicka I, Marcinkiewicz J and Maśliński W: The mechanism of taurine chloramine inhibition of cytokine (interleukin-6, interleukin-8) production by rheumatoid arthritis fibroblast-like synoviocytes. Arthritis Rheum 43: 2169-2177, 2000.

253. Wojtecka-Lukasik E, Gujski M, Roguska K, Maslinska D and Maslinski S: Taurine chloramine modifies adjuvant arthritis in rats. Inflamm Res 54 (Suppl 1): S21-S22, 2005.

254. Verdrengh $\mathrm{M}$ and Tarkowski A: Inhibition of septic arthritis by local administration of taurine chloramine, a product of activated neutrophils. J Rheumatol 32: 1513-1517, 2005.

255. Kontny E, Plebanczyk M, Lisowska B, Olszewska M, Maldyk P and Maslinski W: Comparison of rheumatoid articular adipose and synovial tissue reactivity to proinflammatory stimuli: Contribution to adipocytokine network. Ann Rheum Dis 71: 262-267, 2012

256. Kontny E, Grabowska A, Kowalczewski J, Kurowska M, Janicka I, Marcinkiewicz J and Maśliński W: Taurine chloramine inhibition of cell proliferation and cytokine production by rheumatoid arthritis fibroblast-like synoviocytes. Arthritis Rheum 42: 2552-2560, 1999.

257. Kontny E, Rudnicka W, Kowalczewski J, Marcinkiewicz J and Maslinski W: Selective inhibition of cyclooxygenase 2-generated prostaglandin E2 synthesis in rheumatoid arthritis synoviocytes by taurine chloramine. Arthritis Rheum 48: 1551-1555, 2003.

258. Kontny E, Rudnicka W, Choraży-Massalska M, Marcinkiewicz J and Maśliński W: Taurine chloramine inhibits proliferation of rheumatoid arthritis synoviocytes by triggering a p53-dependent pathway. Inflamm Res 55: 446-455, 2006.

259. Kim KS, Park EK, Ju SM, Jung HS, Bang JS, Kim C, Lee YA, Hong SJ, Lee SH, Yang HI and Yoo MC: Taurine chloramine differentially inhibits matrix metalloproteinase 1 and 13 synthesis in interleukin-1beta stimulated fibroblast-like synoviocytes. Arthritis Res Ther 9: R80, 2007.

260. Wang Y, Cha YN, Kim KS and Kim C: Taurine chloramine inhibits osteoclastogenesis and splenic lymphocyte proliferation in mice with collagen-induced arthritis. Eur J Pharmacol 668: 325-330, 2011.

261. Davies EV, Williams BD and Campbell AK: Synovial fluid polymorphonuclear leucocytes from patients with rheumatoid arthritis have reduced MPO and NADPH-oxidase activity. Br J Rheumatol 29: 415-421, 1990.

262. Kanayama A and Miyamoto Y: Apoptosis triggered by phagocytosis-related oxidative stress through FLIP $_{S}$ down-regulation and JNK activation. J Leukoc Biol 82: 1344-1352, 2007.

263. Emerson DK, McCormick ML, Schmidt JA and Knudson CM: Taurine monochloramine activates a cell death pathway involving Bax and Caspase-9. J Biol Chem 280: 3233-3241, 2005.

264. Vile GF, Rothwell LA and Kettle AJ: Initiation of rapid, P53-dependent growth arrest in cultured human skin fibroblasts by reactive chlorine species. Arch Biochem Biophys 377: $122-128,2000$

265. Klamt F and Shacter E: Taurine chloramine, an oxidant derived from neutrophils, induces apoptosis in human B lymphoma cells through mitochondrial damage. J Biol Chem 280: 21346-21352, 2005.

266. Pilz M, Holinka J, Vavken P, Marian B and Krepler P: Taurine chloramine induces apoptosis in human osteosarcoma cell lines. J Orthop Res 30: 2046-2051, 2012.

267. Gupta R, Seki Y and Yosida J: Role of taurine in spinal cord injury. Curr Neurovasc Res 3: 225-235, 2006.

268. Kulakowski EC and Maturo J: Hypoglycemic properties of taurine: Not mediated by enhanced insulin release. Biochem Pharmacol 33: 2835-2838, 1984

269. McCartney AC and Browne MK: Clinical studies on administration of taurolin in severe sepsis: A preliminary study. Prog Clin Biol Res 272: 361-371, 1988.

270. Staubach KH: Adjuvant therapy of peritonitis with taurolidine. Modulation of mediator liberation. Langenbecks Arch Chir 382 (Suppl 1): S26-S30, 1997 (In German).

271. Wesch G, Petermann C and Linder MM: Drug therapy of peritonitis. 6-year experience with the chemotherapeutic agent and anti-endotoxin taurolin. Fortschr Med 101: 545-550, 1983 (In German). 
272. Jurewitsch B and Jeejeebhoy KN: Taurolidine lock: The key to prevention of recurrent catheter-related bloodstream infections. Clin Nutr 24: 462-465, 2005.

273. Liu Y, Zhang AQ, Cao L, Xia HT and Ma JJ: Taurolidine lock solutions for the prevention of catheter-related bloodstream infections: A systematic review and meta-analysis of randomized controlled trials. PLoS One 8: e79417, 2013.

274. Hayes KC, Stephan ZF and Sturman JA: Growth depression in taurine-depleted infant monkeys. J Nutr 110: 2058-2064, 1980.

275. Braumann C, Winkler G, Rogalla P, Menenakos C and Jacobi CA: Prevention of disease progression in a patient with a gastric cancer-re-recurrence. Outcome after intravenous treatment with the novel antineoplastic agent taurolidine. Report of a case. World J Surg Onco 4: 34, 2006.

276. Stendel R, Picht T, Schilling A, Heidenreich J, Loddenkemper C, Jänisch W and Brock M: Treatment of glioblastoma with intravenous taurolidine. First clinical experience. Anticancer Res 24: 1143-1147, 2004.

277. Teuchner B, Nagl M, Schidlbauer A, Ishiko H, Dragosits E, Ulmer H, Aoki K, Ohno S, Mizuki N, Gottardi W and Larcher C: Tolerability and efficacy of $\mathrm{N}$-chlorotaurine in epidemic keratoconjunctivitis-A double-blind, randomized, phase-2 clinical trial. J Ocul Pharmacol Ther 21: 157-165 2005.

278. Pasich E, Walczewska M, Białecka A, Peruń A, Kasprowicz A and Marcinkiewicz J: Taurine haloamines and biofilm: II Efficacy of taurine bromamine and chlorhexidine against selected microorganisms of oral biofilm. Adv Exp Med Biol 803: $133-143,2015$.

279. Kyriakopoulos A, Logotheti S, Marcinkiewicz J and Nagl M: $\mathrm{N}$-chlorotaurine and $\mathrm{N}$-bromotaurine combination regimen for the cure of valacyclovir-unresponsive herpes zoster comorbidity in a multiple sclerosis patient. Int J Med Pharm Case Rep 7: 1-6, 2016.

280. Kyriakopoulos AM, Nagl M, Orth-Höller D, Marcinkiewicz J, Baliou S and Zoumbourlis V: Successful treatment of a unique chronic multi-bacterial scalp infection with $\mathrm{N}$-chlorotaurine, N-bromotaurine and bromamine T. Access Microbiol: https:// doi.org/10.1099/acmi.0.000126.

281. Zhai X, Zhao J, Wang Y, Wei X, Li G, Yang Y, Chen Z, Bai Y, Wang Q, Chen X and Li M: Bibliometric analysis of global scientific research on lncRNA: A swiftly expanding trend. Biomed Res Int 2018: 7625078, 2018

282. Di Gesualdo F, Capaccioli S and Lulli M: A pathophysiological view of the long non-coding RNA world. Oncotarget 5: 10976-10996, 2014

283. Wapinski O and Chang HY: Long noncoding RNAs and human disease. Trends Cell Biol 21: 354-361, 2011.

284. Thin KZ, Liu X, Feng X, Raveendran S and Tu JC: LncRNADANCR: A valuable cancer related long non-coding RNA for human cancers. Pathol Res Pract 214: 801-805, 2018.

285. Dykes IM and Emanueli C: Transcriptional and post-transcriptional gene regulation by long non-coding RNA. Genomics Proteomics Bioinformatics 15: 177-186, 2017.

286. Dey BK, Mueller AC and Dutta A: Long non-coding RNAs as emerging regulators of differentiation, development, and disease. Transcription 5: e944014, 2014.

287. Li CH and Chen Y: Targeting long non-coding RNAs in cancers: Progress and prospects. Int J Biochem Cell Biol 45: 1895-1910, 2013

288. Herriges MJ, Swarr DT, Morley MP, Rathi KS, Peng T, Stewart KM and Morrisey EE: Long noncoding RNAs are spatially correlated with transcription factors and regulate lung development. Genes Dev 28: 1363-1379, 2014.

289. Kołat D, Hammouz R, Bednarek A and Płuciennik E: Exosomes as carriers transporting long non-coding RNAs: Molecular characteristics and their function in cancer (Review). Mol Med Rep 20: 851-862, 2019

290. Xiao Y, Zhang J and Deng L: Prediction of 1ncRNA-protein interactions using HeteSim scores based on heterogeneous networks. Sci Rep 7: 3664, 2017.

291. Sun B, Liu C, Li H, Zhang L, Luo G, Liang S and Lü M: Research progress on the interactions between long non-coding RNAs and microRNAs in human cancer. Oncol Lett 19: 595-605, 2020.

292. Cesana M, Cacchiarelli D, Legnini I, Santini T, Sthandier O, Chinappi M, Tramontano A and Bozzoni I: A long noncoding RNA controls muscle differentiation by functioning as a competing endogenous RNA. Cell 147: 358-369, 2011.

293. Wang KC and Chang HY: Molecular mechanisms of long noncoding RNAs. Mol Cell 43: 904-914, 2011

294. Mishra K and Kanduri C: Understanding long noncoding RNA and chromatin interactions: What we know so far. Noncoding RNA 5: 54, 2019.
295. Tasharrofi B and Ghafouri-Fard S: Long non-coding RNAs as regulators of the mitogen-activated protein kinase (MAPK) pathway in cancer. Klin Onkol 31: 95-102, 2018

296. Young TL, Matsuda T and Cepko CL: The noncoding RNA taurine upregulated gene 1 is required for differentiation of the murine retina. Curr Biol 15: 501-512, 2005.

297. Santoro M, Nociti V, Lucchini M, De Fino C, Losavio FA and Mirabella M: Expression profile of long non-coding RNAs in serum of patients with multiple sclerosis. J Mol Neurosci 59: $18-23,2016$

298. Zang XJ, Li L, Du X, Yang B and Mei CL: LncRNA TUG1 inhibits the proliferation and fibrosis of mesangial cells in diabetic nephropathy via inhibiting the PI3K/AKT pathway. Eur Rev Med Pharmacol Sci 23: 7519-7525, 2019.

299. Li SY and Susztak K: The long noncoding RNA tug1 connects metabolic changes with kidney disease in podocytes. J Clin Invest 126: 4072-4075, 2016.

300.Gu W, Yuan Y, Wang L, Yang H, Li S, Tang Z and Li Q: Long non-coding RNA TUG1 promotes airway remodelling by suppressing the miR-145-5p/DUSP6 axis in cigarette smoke-induced COPD. J Cell Mol Med 23: 7200-7209, 2019.

301. Long J, Badal SS, Ye Z, Wang Y, Ayanga BA, Galvan DL, Green NH, Chang BH, Overbeek PA and Danesh FR: Long noncoding RNA Tug1 regulates mitochondrial bioenergetics in diabetic nephropathy. J Clin Invest 126: 4205-4218, 2016.

302. Yin DD, Zhang EB, You LH, Wang N, Wang LT, Jin FY, Zhu YN, Cao LH, Yuan QX, De W and Tang W: Downregulation of lncRNA TUG1 affects apoptosis and insulin secretion in mouse pancreatic $\beta$ cells. Cell Physiol Biochem 35: 1892-1904, 2015.

303. Li Z, Shen J, Chan MTV and Wu WKK: TUG1: A pivotal oncogenic long non-coding RNA of human cancers. Cell Prolif 49: 471-475, 2016.

304. Gutschner T and Diederichs S: The hallmarks of cancer: A long non-coding RNA point of view. RNA Biol 9: 703-719, 2012.

305. Shi X, Sun M, Liu H, Yao Y and Song Y: Long non-coding RNAs: A new frontier in the study of human diseases. Cancer Lett 339: 159-166, 2013.

306.Li B, Shen S, Zhang W, Qi T, Hu Q and Cheng Y: Long noncoding RNA TUG1 as a potential novel biomarker for predicting the clinical outcome of cancer patients: A meta-analysis. Clin Lab 64: 2018.

307. van Heesch S, van Iterson M, Jacobi J, Boymans S, Essers PB, de Bruijn E, Hao W, MacInnes AW, Cuppen E and Simonis M: Extensive localization of long noncoding RNAs to the cytosol and mono- and polyribosomal complexes. Genome Biol 15: R6, 2014.

308. Long J, Menggen Q, Wuren Q, Shi Q and Pi X: Long noncoding RNA taurine-upregulated genel (TUG1) promotes tumor growth and metastasis through TUG1/Mir-129-5p/astrocyte-elevated gene-1 (AEG-1) axis in malignant melanoma. Med Sci Monit 24: $1547-1559,2018$

309. Han Y, Liu Y, Gui Y and Cai Z: Long intergenic non-coding RNA TUG1 is overexpressed in urothelial carcinoma of the bladder. J Surg Oncol 107: 555-559, 2013

310. Zhang Q, Geng PL, Yin P, Wang XL, Jia JP and Yao J: Down-regulation of long non-coding RNA TUG1 inhibits osteosarcoma cell proliferation and promotes apoptosis. Asian Pac J Cancer Prev 14: 2311-2315, 2013.

311. Huang MD, Chen WM, Qi FZ, Sun M, Xu TP, Ma P and Shu YQ Long non-coding RNA TUG1 is up-regulated in hepatocellular carcinoma and promotes cell growth and apoptosis by epigenetically silencing of KLF2. Mol Cancer 14: 165, 2015

312. Zhai HY, Sui MH, Yu X, Qu Z, Hu JC, Sun HQ, Zheng HT, Zhou K and Jiang LX: Overexpression of long non-coding RNA TUG1 promotes colon cancer progression. Med Sci Monit 22: 3281-3287, 2016.

313. Li J, Zhang M, An G and Ma Q: LncRNA TUG1 acts as a tumor suppressor in human glioma by promoting cell apoptosis. Exp Biol Med (Maywood) 241: 644-649, 2016.

314. Zhang EB, Yin DD, Sun M, Kong R, Liu XH, You LH, Han L, Xia R, Wang KM, Yang JS, et al: P53-regulated long non-coding RNA TUG1 affects cell proliferation in human non-small cell lung cancer, partly through epigenetically regulating HOXB7 expression. Cell Death Dis 5: e1243, 2014.

315. Kondo Y, Shinjo K and Katsushima K: Long non-coding RNAs as an epigenetic regulator in human cancers. Cancer Sci 108: 1927-1933, 2017

316. Kim KH and Roberts CWM: Targeting EZH2 in cancer. Nat Med 22: 128-134, 2016 
317. Sun CC, Li SJ, Li G, Hua RX, Zhou XH and Li DJ: Long intergenic noncoding RNA 00511 acts as an oncogene in non-small-cell lung cancer by binding to EZH2 and suppressing p57. Mol Ther Nucleic Acids 5: e385, 2016.

318. Cao R, Wang L, Wang H, Xia L, Erdjument-Bromage H, Tempst P, Jones RS and Zhang Y: Role of histone H3 lysine 27 methylation in polycomb-group silencing. Science 298: 1039-1043, 2002.

319. Liu H, Zhou G, Fu X, Cui H, Pu G, Xiao Y, Sun W, Dong X, Zhang L, Cao S, et al: Long noncoding RNA TUG1 is a diagnostic factor in lung adenocarcinoma and suppresses apoptosis via epigenetic silencing of BAX. Oncotarget 8: 101899-101910, 2017.

320. Zhang E, He X, Yin D, Han L, Qiu M, Xu T, Xia R, Xu L, Yin R and De W: Increased expression of long noncoding RNA TUG1 predicts a poor prognosis of gastric cancer and regulates cell proliferation by epigenetically silencing of p57. Cell Death Dis 7: e2109, 2016.

321. Ding B, Lou W, Xu L and Fan W: Non-coding RNA in drug resistance of hepatocellular carcinoma. Biosci Rep 38: BSR20180915, 2018.

322. Yang L, Lin C, Liu W, Zhang J, Ohgi KA, Grinstein JD, Dorrestein PC and Rosenfeld MG: ncRNA- and Pc2 methylation-dependent gene relocation between nuclear structures mediates gene activation programs. Cell 147: 773-788, 2011.

323. Thomson DW and Dinger ME: Endogenous microRNA sponges: Evidence and controversy. Nat Rev Genet 17: 272-283, 2016.

324. Ma L, Bajic VB and Zhang Z: On the classification of long non-coding RNAs. RNA Biol 10: 925-933, 2013.

325. Krol J, Loedige I and Filipowicz W: The widespread regulation of microRNA biogenesis, function and decay. Nat Rev Genet 11: 597-610, 2010.

326. Tsai KW, Wu CW, Hu LY, Li SC, Liao YL, Lai CH, Kao HW, Fang WL, Huang KH, Chan WC and Lin WC: Epigenetic regulation of miR-34b and miR-129 expression in gastric cancer. Int J Cancer 129: 2600-2610, 2011.

327. Ji TT, Huang X, Jin J, Pan SH and Zhuge XJ: Inhibition of long non-coding RNA TUG1 on gastric cancer cell transference and invasion through regulating and controlling the expression of miR-144/c-met axis. Asian Pac J Trop Med 9: 508-512, 2016.

328. Zhang M, Huang S and Long D: MiR-381 inhibits migration and invasion in human gastric carcinoma through downregulating SOX4. Oncol Lett 14: 3760-3766, 2017

329. Li J, Zhang Q, Fan X, Mo W, Dai W, Feng J, Wu L, Liu T, Li S, $\mathrm{Xu} \mathrm{S}$, et al: The long noncoding RNA TUG1 acts as a competing endogenous RNA to regulate the Hedgehog pathway by targeting miR-132 in hepatocellular carcinoma. Oncotarget 8: 65932-65945, 2017.

330. Sun J, Hu J, Wang G, Yang Z, Zhao C, Zhang X and Wang J: LncRNA TUG1 promoted KIAA1199 expression via miR-600 to accelerate cell metastasis and epithelial-mesenchymal transition in colorectal cancer. J Exp Clin Cancer Res 37: 106, 2018.

331. Ma F, Wang S, Cai Q, Jin L, Zhou D, Ding J and Quan ZW: Long non-coding RNA TUG1 promotes cell proliferation and metastasis by negatively regulating miR-300 in gallbladder carcinoma. Biomed Pharmacother 88: 863-869, 2017.

332. Tan J, Qiu K, Li M and Liang Y: Double-negative feedback loop between long non-coding RNA TUG1 and miR-145 promotes epithelial to mesenchymal transition and radioresistance in human bladder cancer cells. FEBS Lett 589 (20 Pt B): 3175-3181, 2015.

333. Cai H, Xue Y, Wang P, Wang Z, Li Z, Hu Y, Li Z, Shang X and Liu Y: The long noncoding RNA TUG1 regulates blood-tumor barrier permeability by targeting miR-144. Oncotarget 6 : 19759-19779, 2015.

334. Xie CH, Cao YM, Huang Y, Shi QW, Guo JH, Fan ZW, Li JG, Chen BW and Wu BY: Long non-coding RNA TUG1 contributes to tumorigenesis of human osteosarcoma by sponging miR-9-5p and regulating POU2F1 expression. Tumor Biol 37: 15031-15041, 2016.

335. Dong R, Liu GB, Liu BH, Chen G, Li K, Zheng S and Dong KR: Targeting long non-coding RNA-TUG1 inhibits tumor growth and angiogenesis in hepatoblastoma. Cell Death Dis 7: e2278, 2016.

336. Cai H, Liu X, Zheng J, Xue Y, Ma J, Li Z, Xi Z, Li Z, Bao M and Liu Y: Long non-coding RNA taurine upregulated 1 enhances tumor-induced angiogenesis through inhibiting microRNA-299 in human glioblastoma. Oncogene 36: 318-331, 2017.

337. Hu Y, Sun X, Mao C, Guo G, Ye S, Xu J, Zou R, Chen J, Wang L, Duan P and Xue X: Upregulation of long noncoding RNA TUG1 promotes cervical cancer cell proliferation and migration. Cancer Med 6: 471-482, 2017.
338. Katsushima K, Natsume A, Ohka F, Shinjo K, Hatanaka A Ichimura N, Sato S, Takahashi S, Kimura H, Totoki Y, et al: Targeting the Notch-regulated non-coding RNA TUG1 for glioma treatment. Nat Commun 7: 13616, 2016.

339. Yun-Bo F, Xiao-Po L, Xiao-Li L, Guo-Long C, Pei Z and Fa-Ming T: LncRNA TUG1 is upregulated and promotes cell proliferation in osteosarcoma. Open Med (Wars) 11: 163-167, 2016

340. Liang S, Zhang S, Wang P, Yang C, Shang C, Yang J and Wang J: LncRNA, TUG1 regulates the oral squamous cell carcinoma progression possibly via interacting with $\mathrm{Wnt} / \beta$-catenin signaling. Gene 608: 49-57, 2017.

341. Tang T, Cheng Y, She Q, Jiang Y, Chen Y, Yang W and Li Y: Long non-coding RNA TUG1 sponges miR-197 to enhance cisplatin sensitivity in triple negative breast cancer. Biomed Pharmacother 107: 338-346, 2018.

342. Xiao CH, Yu HZ, Guo CY, Wu ZM, Cao HY, Li WB and Yuan JF: Long non-coding RNA TUG1 promotes the proliferation of colorectal cancer cells through regulating Wnt $/ \beta$-catenin pathway. Oncol Lett 16: 5317-5324, 2018.

343. Qin CF and Zhao FL: Long non-coding RNA TUG1 can promote proliferation and migration of pancreatic cancer via EMT pathway. Eur Rev Med Pharmacol Sci 21: 2377-2384, 2017.

344.Zhang CG, Yin DD, Sun SY and Han L: The use of lncRNA analysis for stratification management of prognostic risk in patients with NSCLC. Eur Rev Med Pharmacol Sci 21: 115-119, 2017.

345. Lin PC, Huang HD, Chang CC, Chang YS, Yen JC, Lee CC, Chang WH, Liu TC and Chang JG: Long noncoding RNA TUG1 is downregulated in non-small cell lung cancer and can regulate CELF1 on binding to PRC2. BMC Cancer 16: 583, 2016.

346. Baratieh Z, Khalaj Z, Honardoost MA, Emadi-Baygi M, Khanahmad H, Salehi M and Nikpour P: Aberrant expression of PlncRNA-1 and TUG1: Potential biomarkers for gastric cancer diagnosis and clinically monitoring cancer progression. Biomark Med 11: 1077-1090, 2017.

347. Ren K, Li Z, Li Y, Zhang W and Han X: Long noncoding RNA taurine-upregulated gene 1 promotes cell proliferation and invasion in gastric cancer via negatively modulating miRNA-145-5p. Oncol Res 25: 789-798, 2017.

348. Lin YH, Wu MH, Huang YH, Yeh CT and Lin KH: TUG1 is a regulator of AFP and serves as prognostic marker in non-hepatitis B non-hepatitis C hepatocellular carcinoma. Cells 9: 262, 2020.

349. Dai Q, Deng J, Zhou J, Wang Z, Yuan X, Pan S and Zhang HB: Long non-coding RNA TUG1 promotes cell progression in hepatocellular carcinoma via regulating miR-216b-5p/DLX2 axis. Cancer Cell Int 20: 8, 2020.

350. Fang T, Fang Y, Xu X, He M, Zhao Z, Huang P, Yuan F, Guo M, Yang B and Xia J: Actinidia chinensis planch root extract attenuates proliferation and metastasis of hepatocellular carcinoma by inhibiting epithelial-mesenchymal transition. J Ethnopharmacol 231: 474-485, 2019.

351. Huang J, Lu D, Xiang T, Wu X, Ge S, Wang Y, Wang J and Cheng N: MicroRNA-132-3p regulates cell proliferation, apoptosis, migration and invasion of liver cancer by targeting Sox4. Oncol Lett 19: 3173-3180, 2020.

352. Lv J, Kong Y, Gao Z, Liu Y, Zhu P and Yu Z: LncRNA TUG1 interacting with miR-144 contributes to proliferation, migration and tumorigenesis through activating the JAK2/STAT3 pathway in hepatocellular carcinoma. Int J Biochem Cell Biol 101: 19-28, 2018

353. He C, Liu Z, Jin L, Zhang F, Peng X, Xiao Y, Wang X, Lyu Q and Cai XJ: lncRNA TUG1-mediated mir-142-3p downregulation contributes to metastasis and the epithelial-to-mesenchymal transition of hepatocellular carcinoma by targeting ZEB1. Cell Physiol Biochem 48: 1928-1941, 2018.

354. Xie F, Zhang L, Yao Q, Shan L, Liu J, Dong N and Liang J: TUG1 promoted tumor progression by sponging miR-335-5p and regulating CXCR4-mediated infiltration of pro-tumor immunocytes in CTNNB1-mutated hepatoblastoma. Onco Targets Ther 13: 3105-3115, 2020.

355. An N and Cheng D: The long noncoding RNA HOST2 promotes gemcitabine resistance in human pancreatic cancer cells. Pathol Oncol Res 26: 425-431, 2020.

356.Zhao L, Sun H, Kong H, Chen Z, Chen B and Zhou M: The lncrna-TUG1/EZH2 axis promotes pancreatic cancer cell proliferation, migration and EMT phenotype formation through sponging mir-382. Cell Physiol Biochem 42: 2145-2158, 2017.

357. Xu K and Zhang L: Inhibition of TUG1/miRNA-299-3p axis represses pancreatic cancer malignant progression via suppression of the notch1 pathway. Dig Dis Sci 65: 1748-1760, 2020. 
358. Yang F, Li X, Zhang L, Cheng L and Li X: LncRNA TUG1 promoted viability and associated with gemcitabine resistant in pancreatic ductal adenocarcinoma. J Pharmacol Sci 137: 116-121, 2018

359. Burris HA III, Moore MJ, Andersen J, Green MR, Rothenberg ML, Modiano MR, Cripps MC, Portenoy RK, Storniolo AM, Tarassoff $\mathrm{P}$, et al: Improvements in survival and clinical benefit with gemcitabine as first-line therapy for patients with advanced pancreas cancer: A randomized trial. J Clin Oncol 15: 2403-2413, 1997.

360.Lu Y, Tang L, Zhang Z, Li S, Liang S, Ji L, Yang B, Liu Y and Wei W: Long noncoding RNA TUG1/miR-29c axis affects cell proliferation, invasion, and migration in human pancreatic cancer. Dis Markers 2018: 6857042, 2018.

361. Miyamoto Y, Maitra A, Ghosh B, Zechner U, Argani P, Iacobuzio-Donahue CA, Sriuranpong V, Iso T, Meszoely IM, Wolfe MS, et al: Notch mediates TGFo-induced changes in epithelial differentiation during pancreatic tumorigenesis. Cancer Cell 3: 565-576, 2003.

362. Hu H, Zhou L, Awadallah A and Xin W: Significance of Notch1-signaling pathway in human pancreatic development and carcinogenesis. Appl Immunohistochem Mol Morphol 21: 242-247, 2013.

363. Chen X, Qi M, Yang Q and Li JY: MiR-299-3p functions as a tumor suppressor in thyroid cancer by regulating SHOC2. Eur Rev Med Pharmacol Sci 23: 232-240, 2019.

364.Dang S, Zhou J, Wang Z, Wang K, Dai S and He S: MiR-299-3p functions as a tumor suppressor via targeting Sirtuin 5 in hepatocellular carcinoma. Biomed Pharmacother 106: 966-975, 2018.

365. Wang JY, Jiang JB, Li Y, Wang YL and Dai Y: MicroRNA-299-3p suppresses proliferation and invasion by targeting VEGFA in human colon carcinoma. Biomed Pharmacother 93: 1047-1054, 2017

366. Hui B, Xu Y, Zhao B, Ji H, Ma Z, Xu S, He ZY, Wang K and $\mathrm{Lu}$ J: Overexpressed long noncoding RNA TUG1 affects the cell cycle, proliferation, and apoptosis of pancreatic cancer partly through suppressing RND3 and MT2A. Onco Targets Ther 12: 1043-1057, 2019.

367. Zhu Y, Zhou J, Xia H, Chen X, Qiu M, Huang J, Liu S, Tang Q, Lang N, Liu Z, et al: The Rho GTPase RhoE is a p53-regulated candidate tumor suppressor in cancer cells. Int J Oncol 44 896-904, 2014.

368. Poch E, Miñambres R, Mocholí E, Ivorra C, Pérez-Aragó A, Guerri C, Pérez-Roger I and Guasch RM: RhoE interferes with $\mathrm{Rb}$ inactivation and regulates the proliferation and survival of the U87 human glioblastoma cell line. Exp Cell Res 313: 719-731, 2007

369. Wang L, Zhao Z, Feng W, Ye Z, Dai W, Zhang C, Peng J and Wu K: Long non-coding RNA TUG1 promotes colorectal cancer metastasis via EMT pathway. Oncotarget 7: 51713-51719, 2016.

370. Shen X, Hu X, Mao J, Wu Y, Liu H, Shen J, Yu J and Chen W: The long noncoding RNA TUG1 is required for TGF- $\beta$ /TWIST1/EMT-mediated metastasis in colorectal cancer cells. Cell Death Dis 11: 65, 2020.

371. Sun J, Ding C, Yang Z, Liu T, Zhang X, Zhao C and Wang J: The long non-coding RNA TUG1 indicates a poor prognosis for colorectal cancer and promotes metastasis by affecting epithelial-mesenchymal transition. J Transl Med 14: 42, 2016.

372. Fan S, Yang Z, Ke Z, Huang K, Liu N, Fang X and Wang K: Downregulation of the long non-coding RNA TUG1 is associated with cell proliferation, migration, and invasion in breast cancer. Biomed Pharmacother 95: 1636-1643, 2017.

373. Caldon CE, Daly RJ, Sutherland RL and Musgrove EA: Cell cycle control in breast cancer cells. J Cell Biochem 97: 261-274, 2006.

374. Zhao X and Ren G: LncRNA taurine-upregulated gene 1 promotes cell proliferation by inhibiting microRNA-9 in MCF-7 cells. J Breast Cancer 19: 349-357, 2016.

375. Gradia DF, Mathias C, Coutinho R, Cavalli IJ, Ribeiro EMSF and de Oliveira JC: Long non-coding RNA TUG1 expression is associated with different subtypes in human breast cancer. Noncoding RNA 3: 26, 2017.

376. Neuwelt EA, Barnett PA, Bigner DD and Frenkel EP: Effects of adrenal cortical steroids and osmotic blood-brain barrier opening on methotrexate delivery to gliomas in the rodent: The factor of the blood-brain barrier. Proc Natl Acad Sci USA 79: 4420-4423, 1982.

377. Li TH, Zhang JJ, Liu SX and Chen Y: Long non-coding RNA taurine-upregulated gene 1 predicts unfavorable prognosis, promotes cells proliferation, and inhibits cells apoptosis in epithelial ovarian cancer. Medicine (Baltimore) 97: e0575, 2018
378. Iliev R, Kleinova R, Juracek J, Dolezel J, Ozanova Z, Fedorko M, Pacik D, Svoboda M, Stanik M and Slaby O: Overexpression of long non-coding RNA TUG1 predicts poor prognosis and promotes cancer cell proliferation and migration in high-grade muscleinvasive bladder cancer. Tumour Biol 37: 13385-13390, 2016.

379. Guo P, Zhang G, Meng J, He Q, Li Z and Guan Y: Upregulation of long noncoding RNA TUG1 promotes bladder cancer cell proliferation, migration, and invasion by inhibiting miR-29c. Oncol Res 26: 1083-1091, 2018.

380. Liu Q, Liu H, Cheng H, Li Y, Li X and Zhu C: Downregulation of long noncoding RNA TUG1 inhibits proliferation and induces apoptosis through the TUG1/miR-142/ZEB2 axis in bladder cancer cells. Onco Targets Ther 10: 2461-2471, 2017.

381. Jiang $\mathrm{H}, \mathrm{Hu} \mathrm{X}$, Zhang $\mathrm{H}$ and $\mathrm{Li} \mathrm{W}$ : Down-regulation of LncRNA TUG1 enhances radiosensitivity in bladder cancer via suppressing HMGB1 expression. Radiat Oncol 12: 65, 2017.

382. Zhu J, Shi H, Liu H, Wang X and Li F: Long non-coding RNA TUG1 promotes cervical cancer progression by regulating the miR-138-5p-SIRT1 axis. Oncotarget 8: 65253-65264, 2017.

383. Fan M, Li C, He P, Fu Y, Li M and Zhao X: Knockdown of long noncoding RNA-taurine-upregulated gene 1 inhibits tumor angiogenesis in ovarian cancer by regulating leucine-rich a-2-glycoprotein-1. Anticancer Drugs 30: 562-570, 2019.

384. Wang X, Abraham S, McKenzie JAG, Jeffs N, Swire M, Tripathi VB, Luhmann UFO, Lange CAK, Zhai Z, Arthur HM, et al: LRG1 promotes angiogenesis by modulating endothelial TGF- $\beta$ signalling. Nature 499: 306-311, 2013.

385. Goel S, Duda DG, Xu L, Munn LL, Boucher Y, Fukumura D and Jain RK: Normalization of the vasculature for treatment of cancer and other diseases. Physiol Rev 91: 1071-1121, 2011.

386. Meng H, Song Y, Zhu J, Liu Q, Lu P, Ye N, Zhang Z, Pang Y, Qi J and Wu H: LRG1 promotes angiogenesis through upregulating the TGF- $\beta 1$ pathway in ischemic rat brain. Mol Med Rep 14: 5535-5543, 2016.

387. Zhang J, Zhu L, Fang J, Ge Z and Li X: LRG1 modulates epithelial-mesenchymal transition and angiogenesis in colorectal cancer via HIF-1 $\alpha$ activation. J Exp Clin Cancer Res 35: 29, 2016.

388. Baek YY, Cho DH, Choe J, Lee H, Jeoung D, Ha KS, Won MH, Kwon YG and Kim YM: Extracellular taurine induces angiogenesis by activating ERK-, Akt-, and FAK-dependent signal pathways. Eur J Pharmacol 674: 188-199, 2012.

389. Xu T, Liu CL, Li T, Zhang YH and Zhao YH: LncRNA TUG1 aggravates the progression of prostate cancer and predicts the poor prognosis. Eur Rev Med Pharmacol Sci 23: 4698-4705, 2019.

390. Hao SD, Ma JX, Liu Y, Liu PJ and Qin Y: Long non-coding TUG1 accelerates prostate cancer progression through regulating miR-128-3p/YES1 axis. Eur Rev Med Pharmacol Sci 24: 619-632, 2020

391. Jiang L, Wang W, Li G, Sun C, Ren Z, Sheng H, Gao H, Wang C and Yu H: High TUG1 expression is associated with chemotherapy resistance and poor prognosis in esophageal squamous cell carcinoma. Cancer Chemother Pharmacol 78: 333-339, 2016.

392. Xu Y, Wang J, Qiu M, Xu L, Li M, Jiang F, Yin R and Xu L: Upregulation of the long noncoding RNA TUG1 promotes proliferation and migration of esophageal squamous cell carcinoma. Tumor Biol 36: 1643-1651, 2015.

393. Tang Y, Yang P, Zhu Y and Su Y: LncRNA TUG1 contributes to ESCC progression via regulating miR-148a-3p/ MCL-1/Wnt/ $\beta$-catenin axis in vitro. Thorac Cancer 11: 82-94, 2020.

394. Ma B, Li M, Zhang L, Huang M, Lei JB, Fu GH, Liu CX, Lai QW, Chen QQ and Wang YL: Upregulation of long non-coding RNA TUG1 correlates with poor prognosis and disease status in osteosarcoma. Tumor Biol 37: 4445-4455, 2016.

395. Wang H, Yu Y, Fan S and Luo L: Knockdown of Long noncoding RNA TUG1 inhibits the proliferation and cellular invasion of osteosarcoma cells by sponging miR-153. Oncol Res 26: $665-673,2018$

396. Xie C, Chen B, Wu B, Guo J and Cao Y: LncRNA TUG1 promotes cell proliferation and suppresses apoptosis in osteosarcoma by regulating miR-212-3p/FOXA1 axis. Biomed Pharmacother 97: $1645-1653,2018$.

397. Li G, Liu K and Du X: Long Non-Coding RNA TUG1 promotes proliferation and inhibits apoptosis of osteosarcoma cells by sponging miR-132-3p and upregulating SOX4 expression. Yonsei Med J 59: 226-235, 2018

398. Liu Y, Li Y, Liu J, Wu Y and Zhu Q: MicroRNA-132 inhibits cell growth and metastasis in osteosarcoma cell lines possibly by targeting Sox4. Int J Oncol 47: 1672-1684, 2015. 
399. Wang Y, Yang T, Zhang Z, Lu M, Zhao W, Zeng X and Zhang W: Long non-coding RNA TUG1 promotes migration and invasion by acting as a ceRNA of miR-335-5p in osteosarcoma cells. Cancer Sci 108: 859-867, 2017.

400.Li Y, Zhang T, Zhang Y, Zhao X and Wang W: Targeting the FOXM1-regulated long noncoding RNA TUG1 in osteosarcoma. Cancer Sci 109: 3093-3104, 2018.

401. Cao J, Han X, Qi X, Jin X and Li X: TUG1 promotes osteosarcoma tumorigenesis by upregulating EZH2 expression via miR-144-3p. Int J Oncol 51: 1115-1123, 2017.

402. Yu X, Hu L, Li S, Shen J, Wang D, Xu R and Yang H: Long non-coding RNA Taurine upregulated gene 1 promotes osteosarcoma cell metastasis by mediating HIF-1 $\alpha$ via miR-143-5p. Cell Death Dis 10: 280, 2019.

403. Li Q, Song W and Wang J: TUG1 confers Adriamycin resistance in acute myeloid leukemia by epigenetically suppressing miR-34a expression via EZH2. Biomed Pharmacother 109: 1793-1801, 2019.

404. Isin M, Ozgur E, Cetin G, Erten N, Aktan M, Gezer U and Dalay $\mathrm{N}$ : Investigation of circulating lncRNAs in B-cell neoplasms. Clin Chim Acta 431: 255-259, 2014.

405. Hanahan D and Weinberg RA: Hallmarks of cancer: The next generation. Cell 144: 646-674, 2011.

406. Yang L, Moss T, Mangala LS, Marini J, Zhao H, Wahlig S, Armaiz-Pena G, Jiang D, Achreja A, Win J, et al: Metabolic shifts toward glutamine regulate tumor growth, invasion and bioenergetics in ovarian cancer. Mol Syst Biol 10: 728, 2014

407. Wang J, Xie H, Ling Q, Lu D, Lv Z, Zhuang R, Liu Z, Wei X, Zhou L, Xu X and Zheng S: Coding-noncoding gene expression in intrahepatic cholangiocarcinoma. Transl Res 168: 107-121, 2016.

408.Zeng B, Ye H, Chen J, Cheng D, Cai C, Chen G, Chen X, $\mathrm{Xin} \mathrm{H}$, Tang $\mathrm{C}$ and Zeng J: LncRNA TUG1 sponges miR-145 to promote cancer progression and regulate glutamine metabolism via Sirt3/GDH axis. Oncotarget 8: 113650-113661, 2017.

409. Han X, Yang Y, Sun Y, Qin L and Yang Y: LncRNA TUG1 affects cell viability by regulating glycolysis in osteosarcoma cells. Gene 674: 87-92, 2018.

410. Kunej T, Obsteter J, Pogacar Z, Horvat S and Calin GA: The decalog of long non-coding RNA involvement in cancer diagnosis and monitoring. Crit Rev Clin Lab Sci 51: 344-357, 2014.

411. Dutour A, Leclers D, Monteil J, Paraf F, Charissoux JL, Rousseau R and Rigaud M: Non-invasive imaging correlates with histological and molecular characteristics of an osteosarcoma model: Application for early detection and follow-up of MDR phenotype. Anticancer Res 27 (6B): 4171-4178, 2007.

412. Fidler IJ: The biology of brain metastasis: Challenges for therapy. Cancer J 21: 284-293, 2015.

413. La Porta CA: Drug resistance in melanoma: New perspectives. Curr Med Chem 14: 387-391, 2007.

414. Liu YY, Han TY, Giuliano AE and Cabot MC: Ceramide glycosylation potentiates cellular multidrug resistance. FASEB J 15: 719-730, 2001.
415. Synold TW, Dussault I and Forman BM: The orphan nuclear receptor SXR coordinately regulates drug metabolism and efflux. Nat Med 7: 584-590, 2001.

416. Gottesman MM: Mechanisms of cancer drug resistance. Annu Rev Med 53: 615-627, 2002.

417. Liu Z and Zhang H: LncRNA plasmacytoma variant translocation 1 is an oncogene in bladder urothelial carcinoma. Oncotarget 8: 64273-64282, 2017

418. Xie D, Zhang H, Hu X and Shang C: Knockdown of long non-coding RNA Taurine Up-Regulated 1 inhibited doxorubicin resistance of bladder urothelial carcinoma via Wnt/ $\beta$-catenin pathway. Oncotarget 8: 88689-88696, 2017.

419. Li C, Gao Y, Li Y and Ding D: TUG1 mediates methotrexate resistance in colorectal cancer via miR-186/CPEB2 axis. Biochem Biophys Res Commun 491: 552-557, 2017.

420. Gu L, Li Q, Liu H, Lu X and Zhu M: Long noncoding RNA TUG1 promotes autophagy-associated paclitaxel resistance by sponging miR-29b-3p in ovarian cancer cells. OncoTargets Ther 13: 2007-2019, 2020.

421. Xi G, Hu X, Wu B, Jiang H, Young CY, Pang Y and Yuan H: Autophagy inhibition promotes paclitaxel-induced apoptosis in cancer cells. Cancer Lett 307: 141-148, 2011.

422. Wei X, Zhou Y, Qiu J, Wang X, Xia Y and Sui L: Low expression of TUG1 promotes cisplatin sensitivity in cervical cancer by activating the MAPK pathway. J BUON 24: 1020-1026, 2019.

423. Köberle B, Tomicic MT, Usanova S and Kaina B: Cisplatin resistance: Preclinical findings and clinical implications. Biochim Biophys Acta 1806: 172-182, 2010.

424. Niu Y, Ma F, Huang W, Fang S, Li M, Wei T and Guo L: Long non-coding RNA TUG1 is involved in cell growth and chemoresistance of small cell lung cancer by regulating LIMK $2 b$ via EZH2. Mol Cancer 16: 5, 2017.

425. Xu C, Guo Y, Liu H, Chen G, Yan Y and Liu T: TUG1 confers cisplatin resistance in esophageal squamous cell carcinoma by epigenetically suppressing PDCD4 expression via EZH2. Cell Biosci 8: 61, 2018.

426. Jemal A, Bray F, Center MM, Ferlay J, Ward E and Forman D: Global cancer statistics. CA Cancer J Clin 61: 69-90, 2011.

427. Liu Q, Sun S, Yu W, Jiang J, Zhuo F, Qiu G, Xu S and Jiang X: Altered expression of long non-coding RNAs during genotoxic stress-induced cell death in human glioma cells. J Neurooncol 122: 283-292, 2015.

428. Wang X, Chen X, Zhang D, Yang G, Yang Z, Yin Z and Zhao S: Prognostic and clinicopathological role of long non-coding RNA taurine upregulated 1 in various human malignancies: A systemic review and meta-analysis. Tumor Biol 39: 1010428317714361, 2017.

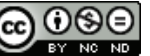

This work is licensed under a Creative Commons Attribution-NonCommercial-NoDerivatives 4.0 International (CC BY-NC-ND 4.0) License. 